\title{
A Combined Experimental and Computational Investigation of the Mechanism of Nickel-Catalyzed Three Component Addition Processes
}

\author{
Hrant Hratchian, Sanjoy K. Chowdhury, Víctor M. Gutiérrez-García, Kande K. D. Amarasinghe, Mary \\ Jane Heeg, ${ }^{\#}$ H. Bernhard Schlegel, ${ }^{*}$ John Montgomery*
}

Department of Chemistry, Wayne State University, Detroit, MI 48202-3489

\section{Experimental Section}

All reagents were used as received unless otherwise noted. Tetrahydrofuran (THF) was freshly distilled from sodium/benzophenone ketyl. TMEDA (tetramethylethylenediamine) was distilled from calcium hydride. Benzaldehyde and methyl iodide were freshly distilled. Methylene chloride, hexane and pentane were distilled from calcium hydride, and further degassed by the freeze-pump-thaw technique. $\mathrm{Ni}(\mathrm{COD})_{2}$ was stored and weighed in an inert atmosphere glovebox. All reactions were conducted in flame-dried glassware on a Schlenk line under an argon atmosphere.

\section{Complex 14a}

TMEDA $(75 \mu 1,0.500 \mathrm{mmol})$ was added dropwise to a $0{ }^{\circ} \mathrm{C}$ solution of $\mathrm{Ni}(\mathrm{COD}) 2(0.138 \mathrm{~g}, 0.500$ mmol) in THF $(10 \mathrm{~mL})$. The resulting yellow solution was stirred at $0{ }^{\circ} \mathrm{C}$ for $5 \mathrm{~min}$. To this solution was added dropwise a $0{ }^{\circ} \mathrm{C}$ solution of 13a $(99 \mathrm{mg}, 0.500 \mathrm{mmol})$ in $5 \mathrm{~mL}$ THF, and the resulting red solution was stirred at $0{ }^{\circ} \mathrm{C}$ for $2 \mathrm{~h} .5 \mathrm{~mL}$ THF was removed under vacuum and the remaining solution was filtered through a filter paper-tipped cannula. The red solution was further concentrated to $c a$. 5 mL. $15 \mathrm{~mL}$ hexane was then slowly added, and the mixture was kept at $-30{ }^{\circ} \mathrm{C}$ for $24 \mathrm{~h}$ to induce crystallization. The precipitate was isolated by removal of the supernatant through a filter paper-tipped 
cannula while maintaining the mixture at $0{ }^{\circ} \mathrm{C}$. The red crystalline solid was washed with hexane $(3 \times 3$ $\mathrm{mL}$ ) and dried in vacuo to yield 14a (95 mg, $51 \%$ ). This complex crystallized with 0.25 equiv of $\mathrm{THF} /$ molecule of $\mathbf{1 4 a}$ in the lattice (confirmed by ${ }^{1} \mathrm{H}$ NMR integration and consistent with elemental analysis). In the crystallographic analysis, the lattice contained a disordered and ill-defined solvent region that could not be reasonably assigned in the crystallographic model. mp $125-126{ }^{\circ} \mathrm{C} \mathrm{dec} ;{ }^{1} \mathrm{H}$ NMR $\left(500 \mathrm{MHz}, \mathrm{CD}_{2} \mathrm{Cl}_{2}\right) \delta 7.94(\mathrm{~d}, J=7.0 \mathrm{~Hz}, 2 \mathrm{H}), 7.23(\mathrm{t}, J=7.5 \mathrm{~Hz}, 2 \mathrm{H}), 7.04(\mathrm{t}, J=7.5 \mathrm{~Hz}$, $1 \mathrm{H}), 5.92-5.94(\mathrm{~m}, 1 \mathrm{H}), 5.55(\mathrm{dd}, J=6.0,2.5 \mathrm{~Hz}, 1 \mathrm{H}), 3.48(\mathrm{dd}, J=6.0,3.5 \mathrm{~Hz}, 1 \mathrm{H}), 2.51-2.59(\mathrm{~m}$, 2H), $2.47(\mathrm{~s}, 3 \mathrm{H}), 2.42(\mathrm{~s}, 3 \mathrm{H}), 2.32-2.37(\mathrm{~m}, 1 \mathrm{H}), 2.45(\mathrm{~s}, 3 \mathrm{H}), 2.19-2.23(\mathrm{~m}, 1 \mathrm{H}), 2.07(\mathrm{dt}, J=13.0$, $4.0 \mathrm{~Hz}, 1 \mathrm{H}), 1.92-1.99(\mathrm{~m}, 1 \mathrm{H}), 1.84(\mathrm{~m}, 2 \mathrm{H}), 1.75(\mathrm{~s}, 3 \mathrm{H}), 1.69-1.73(\mathrm{~m}, 1 \mathrm{H}), 1.61-1.67(\mathrm{~m}, 1 \mathrm{H}) ;{ }^{13} \mathrm{C}$ NMR $\left(125 \mathrm{MHz}, \mathrm{CD}_{2} \mathrm{Cl}_{2}\right) \delta 152.5,147.9,146.2,128.2,127.5,123.0,119.9,100.7,61.5,55.9,50.6$, 48.0, 46.7, 45.1, 41.8, 35.2, 30.9, 26.2; IR (nujol) 1577, $1429 \mathrm{~cm}^{-1}$; Anal. calcd for $\mathrm{C}_{20} \mathrm{H}_{30} \mathrm{ON}_{2} \mathrm{Ni}_{\mathrm{C}} \mathrm{C}$, 64.49; H, 8.13; N, 7.52; calcd for $\mathrm{C}_{20} \mathrm{H}_{30} \mathrm{ON}_{2} \mathrm{Ni} \cdot 1 / 4$ THF: C, 64.58; H, 8.27; N, 7.18. Found: C, 64.41; H, 8.81; N, 7.30.

\section{Complex 14b}

A solution of $2,2^{\prime}$ bipyridine $(78 \mathrm{mg}, 0.500 \mathrm{mmol})$ in $5 \mathrm{~mL}$ THF was transferred by cannula to a solution of $\mathrm{Ni}(\mathrm{COD})_{2}(0.138 \mathrm{~g}, 0.500 \mathrm{mmol})$ in $5 \mathrm{~mL}$ THF. The purple solution was stirred at $\mathrm{rt}$ for 5 $10 \mathrm{~min}$ and was cooled to $0{ }^{\circ} \mathrm{C}$. This solution was added to a $0{ }^{\circ} \mathrm{C}$ solution of $13 \mathbf{a}(99 \mathrm{mg} .0 .500 \mathrm{mg})$ in $5 \mathrm{~mL}$ THF. The dark purple solution was stirred at $0{ }^{\circ} \mathrm{C}$ for $2 \mathrm{~h}$. Volatiles were removed in vacuo, and the residue was dissolved in $30 \mathrm{~mL} \mathrm{CH}_{2} \mathrm{Cl}_{2}$ and filtered through a $2 \mathrm{~cm}$ pad of celite in an airless filtration device. The purple solution was concentrated under reduced pressure to $c a$. $10 \mathrm{~mL}$, and 25 $\mathrm{mL}$ of hexane was slowly added. The mixture was kept at $-30{ }^{\circ} \mathrm{C}$ for $24 \mathrm{~h}$ to induce crystallization. The crystals were isolated by removal of the supernatant through a filter paper-tipped cannula while 
maintaining the mixture at $0{ }^{\circ} \mathrm{C}$. The dark purple crystalline solid was washed with hexane $(2 \times 5 \mathrm{~mL})$ and was dried in vacuo to yield $14 \mathbf{b}(0.111 \mathrm{~g}, 54 \%) . \mathrm{mp} 184-186{ }^{\circ} \mathrm{C} \mathrm{dec} ;{ }^{1} \mathrm{H}$ NMR $(400 \mathrm{MHz}$, $\left.\mathrm{CD}_{2} \mathrm{Cl}_{2}\right) \delta 8.78-8.79(\mathrm{~m}, 1 \mathrm{H}), 8.60(\mathrm{~d}, J=4.8 \mathrm{~Hz}, 1 \mathrm{H}), 8.13-8.15(\mathrm{~m}, 2 \mathrm{H}), 7.96-8.00(\mathrm{~m}, 1 \mathrm{H}), 7.82(\mathrm{~d}$, $J=7.6 \mathrm{~Hz}, 1 \mathrm{H}), 7.77(\mathrm{t}, J=6.8 \mathrm{~Hz}, 1 \mathrm{H}), 7.67(\mathrm{~d}, J=7.2 \mathrm{~Hz}, 1 \mathrm{H}), 7.59(\mathrm{t}, J=6.6 \mathrm{~Hz}, 1 \mathrm{H}), 7.11-7.19$ $(\mathrm{m}, 3 \mathrm{H}), 7.01(\mathrm{t}, J=7.2 \mathrm{~Hz}, 1 \mathrm{H}), 6.07(\mathrm{dd}, J=6.4,2.4 \mathrm{~Hz}, 1 \mathrm{H}), 4.99(\mathrm{t}, J=3.3 \mathrm{~Hz}, 1 \mathrm{H}), 3.71(\mathrm{dd}, J$ $=5.8,4.2 \mathrm{~Hz}, 1 \mathrm{H}), 2.67-2.76(\mathrm{~m}, 1 \mathrm{H}), 2.52-2.58(\mathrm{~m}, 1 \mathrm{H}), 1.87-1.96(\mathrm{~m}, 1 \mathrm{H}), 1.63-1.78(\mathrm{~m}, 3 \mathrm{H}) ;{ }^{13} \mathrm{C}$ NMR $\left(100 \mathrm{MHz}, \mathrm{CD}_{2} \mathrm{Cl}_{2}\right) \delta 152.6,152.1,147.6,138.4,136.5,128.8,127.3,126.1,125.9,123.5$, 120.7, 119.8, 101.9, 41.4, 34.6, 31.4, 26.3; IR (nujol) 1576, 1443, $1295 \mathrm{~cm}^{-1}$.

\section{Complex 16}

A solution of 2,2' bipyridine $(76 \mathrm{mg}, 0.48 \mathrm{mmol})$ in THF $(8 \mathrm{~mL}, 0.06 \mathrm{M})$ was added dropwise by cannula to $\mathrm{Ni}(\mathrm{COD})_{2}(0.134 \mathrm{~g}, 0.480 \mathrm{mmol})$ at $0{ }^{\circ} \mathrm{C}$. The resulting purple solution was stirred for $5-10$ min. A $0{ }^{\circ} \mathrm{C}$ solution of $15(0.148 \mathrm{~g}, 0.480 \mathrm{mmol})$ in THF $(8 \mathrm{~mL}, 0.06 \mathrm{M})$ was added dropwise, and resulting deep red reaction mixture was stirred for $2 \mathrm{~h}$ at $0{ }^{\circ} \mathrm{C}$. THF was removed under reduced pressure to afford a red powder, and the solid was redissolved in $\mathrm{CH}_{2} \mathrm{Cl}_{2}(10 \mathrm{~mL})$. The mixture was filtered through a filter paper tipped cannula and concentrated to $c a .5 \mathrm{~mL}$. Pentane $(4 \mathrm{~mL})$ was added dropwise, and the mixture was cooled to $-30{ }^{\circ} \mathrm{C}$ for 3 days to induce crystallization. The supernatant was removed via cannula while maintaining the mixture at $0{ }^{\circ} \mathrm{C}$. The remaining red crystals were washed with pentane $(5 \mathrm{~mL})$ and dried in vacuo to yield complex $16(0.224 \mathrm{~g}, 80 \%)$. The complex crystallized with 0.75 equiv of $\mathrm{CH}_{2} \mathrm{Cl}_{2} /$ molecule of 16 in the lattice (confirmed by elemental analysis and by X-ray crystallographic analysis). Methylene chloride was disordered in the crystallographic analysis. mp $161-162{ }^{\circ} \mathrm{C} \mathrm{dec} ;{ }^{1} \mathrm{H} \mathrm{NMR}\left(500 \mathrm{MHz}, \mathrm{CD}_{2} \mathrm{Cl}_{2}\right) \delta 8.72(\mathrm{~d}, J=4.5 \mathrm{~Hz}, 2 \mathrm{H}), 7.47$ (br s, 4H), $7.21-7.24(\mathrm{~m}, 6 \mathrm{H}), 7.14(\mathrm{t}, J=7.0 \mathrm{~Hz}, 2 \mathrm{H}), 6.95-6.98(\mathrm{~m}, 4 \mathrm{H}), 5.12(\mathrm{~d}, J=11.5 \mathrm{~Hz}, 2 \mathrm{H}), 3.86$ 
$(\mathrm{dt}, J=11.5,5.5 \mathrm{~Hz}, 2 \mathrm{H}), 1.90(\mathrm{br} \mathrm{s}, 2 \mathrm{H}), 1.74-1.76(\mathrm{~m}, 2 \mathrm{H}), 1.11(\mathrm{br} \mathrm{s}, 2 \mathrm{H})$. The ${ }^{1} \mathrm{H}$ NMR of this complex displayed signals at $\delta 5.60$ and 2.40 whose integrals varied from sample to sample. The origin of these signals was not identified. ${ }^{13} \mathrm{C} \mathrm{NMR}\left(125 \mathrm{MHz}, \mathrm{CD}_{2} \mathrm{Cl}_{2}\right) \delta 188.5,151.4,150.5,140.6$, $135.5,129.7,127.5,126.4,124.9,120.3,63.4,58.6,30.1,23.9$, IR (nujol) $1611,1590,1548 \mathrm{~cm}^{-1}$, Anal. calcd for $\mathrm{C}_{31} \mathrm{H}_{28} \mathrm{O}_{2} \mathrm{~N}_{2} \mathrm{Ni} \cdot 3 / 4 \mathrm{CH}_{2} \mathrm{Cl}_{2}$ : C, 63.28; $\mathrm{H}, 5.02 ; \mathrm{N}, 4.65$ found: $\mathrm{C}, 63.07 ; \mathrm{H}, 4.90 ; \mathrm{N}$, 4.71.

\section{Complex 18}

Following the above mentioned procedure, 2,2' bipyridine (70 $\mathrm{mg}, 0.45 \mathrm{mmol}$, in $0.06 \mathrm{M}$ THF), $\mathrm{Ni}(\mathrm{COD})_{2}(124 \mathrm{mg}, 0.45 \mathrm{mmol})$, and $17(0.102 \mathrm{~g}, 0.450 \mathrm{mmol}$, in $0.06 \mathrm{M} \mathrm{THF})$ was stirred for $2 \mathrm{~h}$ at 0 ${ }^{\circ} \mathrm{C}$. The deep red solution was concentrated under reduced pressure to $c a .8 \mathrm{~mL}$, and filtered through a filter paper-tipped cannula and further concentrated to $c a .5 \mathrm{~mL}$ at $0{ }^{\circ} \mathrm{C}$. Pentane $(3 \mathrm{~mL})$ was added dropwise, and the mixture was cooled to $-30{ }^{\circ} \mathrm{C}$ for 3 days to induce crystallization. The supernatant was removed via cannula and the reddish black crystals were washed with pentane $(5 \mathrm{~mL})$, while maintaining the mixture at $0{ }^{\circ} \mathrm{C}$. The red crystals were dried in vacuo to afford complex $\mathbf{1 8}(72 \mathrm{mg}, 40$ \%). mp 154-156 ${ }^{\circ} \mathrm{C} \mathrm{dec}$; IR (nujol) 1583, $1548 \mathrm{~cm}^{-1}$. This complex was highly unstable, and satisfactory NMR data and elemental analysis could not be obtained.

\section{2-(2-Oxo-phenylethyl)-(E)-1-(1-phenylethylidine)cyclopentane (19a)}

MeLi $\left(228 \mu \mathrm{l}, 0.364 \mathrm{mmol}, 1.6 \mathrm{M}\right.$ in $\left.\mathrm{Et}_{2} \mathrm{O}\right)$ was added dropwise to a $0{ }^{\circ} \mathrm{C}$ solution of $\mathrm{ZnCl} 2(33 \mathrm{mg}$, $0.243 \mathrm{mmol}$ ) in $3 \mathrm{~mL}$ THF. The solution was stirred at $0{ }^{\circ} \mathrm{C}$ for $10 \mathrm{~min}$ and was transferred to a $0{ }^{\circ} \mathrm{C}$ solution of $14 \mathrm{a}$ (30 mg, $0.081 \mathrm{mmol}$ ) in $3 \mathrm{~mL}$ THF. The dark red solution was stirred at $0{ }^{\circ} \mathrm{C}$ for $2 \mathrm{~h}$. The reaction mixture was quenched with $\mathrm{pH} 8$ buffered $\mathrm{NH}_{4} \mathrm{Cl} / \mathrm{NH}_{4} \mathrm{OH}$ and extracted with ether (x3). 
The combined organic layers were washed with brine, dried over $\mathrm{MgSO}_{4}$, filtered, and concentrated. The residue was chromatographed on silica gel (15: 1 hexane/EtOAc) to afford 19a (12.4 mg, $71 \%)$ as a colorless oil. ${ }^{1} \mathrm{H} \mathrm{NMR}\left(400 \mathrm{MHz}, \mathrm{CDCl}_{3}\right) \delta 9.84(\mathrm{dd}, J=2.8,1.8 \mathrm{~Hz}, 1 \mathrm{H}), 7.31$ (t, $\left.J=7.6 \mathrm{~Hz}, 2 \mathrm{H}\right)$, 7.17-7.22 (m, 3H), 3.26-3.04 (m, 1H), $2.68(\mathrm{~m}, 1 \mathrm{H}), 2.50$ (ddd, $J=17.0,10.4,2.6 \mathrm{~Hz}, 1 \mathrm{H}), 2.13-2.30$ $(\mathrm{m}, 2 \mathrm{H}), 2.02(\mathrm{~s}, 3 \mathrm{H}), 1.92-1.99(\mathrm{~m}, 1 \mathrm{H}), 1.62-1.72(\mathrm{~m}, 1 \mathrm{H}), 1.49-1.61(\mathrm{~m}, 2 \mathrm{H}) ;{ }^{13} \mathrm{C} \mathrm{NMR}(100 \mathrm{MHz}$, $\left.\mathrm{CDCl}_{3}\right) \delta 202.6,144.8,142.1,128.5,128.2,127.9,126.4,48.5,36.6,32.7,31.9,24.8,21.2$; IR (film) 3077, 3049, 3013, 2950, 2866, 1717, 1485, $1435 \mathrm{~cm}^{-1}$; HRMS(EI) $\mathrm{m} / z$ calcd for $\mathrm{C}_{15} \mathrm{H}_{18} \mathrm{O}: 214.1358$ found: $214.1358\left(\mathrm{M}^{+}\right)$.

\section{$\left(3 R^{*}, 5 R^{*}\right)-2-($ phenyl)bicyclo[3.3.0]oct-1-en-3-ol (20)}

Complex 14a (29 mg, $0.078 \mathrm{mmol})$ was dissolved in $6 \mathrm{~mL}$ THF $(0.01 \mathrm{M})$ and was cooled to $0{ }^{\circ} \mathrm{C}$. The mixture was quenched with $2.5 \mathrm{~mL} \mathrm{pH} 8$ buffered $\mathrm{NH} 4 \mathrm{Cl} / \mathrm{NH} 4 \mathrm{OH}$ and stirred for 10 min at $0{ }^{\circ} \mathrm{C}$ followed by $15 \mathrm{~min}$ at $\mathrm{rt}$. The reaction mixture was extracted with ether $(5 \mathrm{~mL} \mathrm{X} 3)$. The combined organic layers were washed with brine, dried over $\mathrm{MgSO} 4$, filtered and concentrated. The residue was chromatographed on silica gel (4:1 hexane/ethyl acetate) to afford $\mathbf{2 0}(14.7 \mathrm{mg}, 91.2 \%)$ as a colorless oil which solidifies on standing. Spectroscopic data were identical to that previously reported. ${ }^{9 b}$

\section{$\left(3 R^{*}, 4 R^{*}, 5 R^{*}\right)-4-M e t h y l-2-(p h e n y l) b i c y c l o[3.3 .0]$ oct-1-en-3-ol (21)}

To a $0{ }^{\circ} \mathrm{C}$ solution of $14 \mathbf{a}(23 \mathrm{mg}, 0.062 \mathrm{mmol})$ in THF $(3 \mathrm{~mL})$ was added $\mathrm{MeI}(12 \mu \mathrm{l}, 0.185 \mathrm{mmol})$. The resulting green solution was stirred at $0{ }^{\circ} \mathrm{C}$ for $1.5 \mathrm{~h}$. The reaction mixture was quenched with $\mathrm{pH}$ 8 buffered $\mathrm{NH}_{4} \mathrm{Cl} / \mathrm{NH}_{4} \mathrm{OH}$ and extracted with ether (x3). The combined organic layers were washed with brine, dried over $\mathrm{MgSO}$, filtered, and concentrated. The residue was chromatographed on silica 
gel (9:1 hexane/EtOAc) to give $21(9.2 \mathrm{mg}, 69 \%)$ as a white crystalline solid. Spectroscopic data were identical to that previously reported. ${ }^{9 b}$

\section{$\left(3 R^{*}, 4 R^{*}, 5 R^{*}\right)-2-P h e n y l-4-\left[\left(S^{*}\right)-p h e n y l m e t h a n o l\right] b i c y c l o[3.3 .0]$ oct-1-en-3-ol (22)}

To a $0{ }^{\circ} \mathrm{C}$ solution of $\mathbf{1 4 a}(20 \mathrm{mg}, 0.054 \mathrm{mmol})$ in THF $(3 \mathrm{ml})$ was added PhCHO $(16 \mu 1,0.161 \mathrm{mmol})$. The resulting red solution was stirred at $\mathrm{rt}$ for $12 \mathrm{~h}$. The reaction mixture was quenched with $\mathrm{pH} 8$ buffered $\mathrm{NH}_{4} \mathrm{Cl} / \mathrm{NH}_{4} \mathrm{OH}$ and extracted with ether (x3). The combined organic layers were washed with brine, dried over $\mathrm{MgSO}_{4}$, filtered, and concentrated. The residue was chromatographed on silica gel (6:1 hexane/EtOAc) to afford $22(11.2 \mathrm{mg}, 68 \%)$ as a white crystalline solid. Spectroscopic data were identical to that previously reported. ${ }^{9 b}$

\section{Scheme 7 experimental}

A 2.0 M toluene solution of Me2Zn $(0.61 \mathrm{mmol}, 0.3 \mathrm{~mL})$ was added to a solution of complex 14a (15 $\mathrm{mg}, 0.04 \mathrm{mmol}, 20 \mathrm{~mol} \%$ ) in $2.3 \mathrm{~mL} \mathrm{THF}$, and the mixture was stirred for $5 \mathrm{~min}$ at $0{ }^{\circ} \mathrm{C}$. The deep red reaction mixture was transferred dropwise $(1 \mathrm{~min})$ by cannula to a $0{ }^{\circ} \mathrm{C}$ solution of $\mathbf{1 3 b}(52 \mathrm{mg}, 0.2$ mmol) in $1 \mathrm{~mL}$ THF. The resulting reaction mixture was stirred for $5 \mathrm{~min}$ at $0{ }^{\circ} \mathrm{C}$ and quenched with 2 $\mathrm{mL}$ pH 8 buffered $\mathrm{NH} 4 \mathrm{Cl} / \mathrm{NH} 4 \mathrm{OH}$. The reaction mixture was extracted with $\mathrm{Et} 2 \mathrm{O}(10 \mathrm{~mL} \times 3)$, the combined organic layers were washed with brine, dried over $\mathrm{MgSO} 4$, filtered, and concentrated under reduced pressure. GCMS analysis of the crude reaction mixture revealed a $2.3: 97.7$ ratio of 19a : 19b. The crude reaction mixture was chromatographed on silica gel ( $9: 1$ hexane/ethyl acetate) to afford an inseparable mixture of monocyclic products 19a and 19b (41.4 mg, $73 \%$ total) and pure compound $20(6.6 \mathrm{mg}, 82 \%)$.

Compound 19b: ${ }^{1} \mathrm{H}$ NMR $\left(500 \mathrm{MHz}, \mathrm{CDCl}_{3}\right) \delta 9.84(\mathrm{~s}, 1 \mathrm{H}), 7.32(\mathrm{~d}, \mathrm{~J}=8.5 \mathrm{~Hz}, 2 \mathrm{H}), 7.13(\mathrm{~d}, \mathrm{~J}=8$ $\mathrm{Hz}, 2 \mathrm{H}), 3.28(\mathrm{~m}, 1 \mathrm{H}), 2.67(\mathrm{dd}, \mathrm{J}=3.5,16.5 \mathrm{~Hz}, 1 \mathrm{H}), 2.49(\mathrm{ddd}, \mathrm{J}=2.5,10.5,17 \mathrm{~Hz}, 1 \mathrm{H}), 2.19-2.31$ 
(m, 2H), $2.01(\mathrm{~s}, 3 \mathrm{H}), 1.93-1.99(\mathrm{~m}, 1 \mathrm{H}), 1.62-1.71(\mathrm{~m}, 1 \mathrm{H}), 1.49-1.59(\mathrm{~m}, 2 \mathrm{H}), 1.33(\mathrm{~s}, 9 \mathrm{H}) ;{ }^{13} \mathrm{C}$ NMR (125 MHz, $\left.\mathrm{CDCl}_{3}\right) \delta 202.7,149.1,141.8,141.7,128.2,127.6,125.0,48.8,36.6,34.7,32.7$, 32.1, 31.6, 24.9, 21.1; IR (Neat) 3081, 3027, 2959, 2867, 1724, 1509, $1462 \mathrm{~cm}^{-1}$ HRMS (EI) $\mathrm{m} / z$ calcd for C19H26O: 270.1984 found: 270.1988.

\section{Scheme 8 (production of 19a)}

A solution of bis- $(S)$-oxazoline $23(5.3 \mathrm{mg}, 0.02 \mathrm{mmol})$ in $1 \mathrm{~mL}$ THF was added to a solution of $\mathrm{Ni}(\mathrm{COD}) 2(5.5 \mathrm{mg}, 0.02 \mathrm{mmol})$ in $1 \mathrm{~mL}$ THF and the mixture was stirred for $5 \mathrm{~min}$. This yellow solution was transferred by cannula to a $0{ }^{\circ} \mathrm{C} \mathrm{ZnMe} 2(0.3 \mathrm{~mL}$ of a $2.0 \mathrm{M}$ toluene solution, $0.6 \mathrm{mmol})$ solution in $2 \mathrm{~mL}$ THF. The resulting mixture was transferred dropwise by cannula to a $0{ }^{\circ} \mathrm{C}$ solution of substrate 13a (40 mg, $0.2 \mathrm{mmol}$ ) in $2 \mathrm{~mL}$ THF. The red reaction mixture was stirred $5 \mathrm{~min}$, then it was quenched by adding $2 \mathrm{~mL} \mathrm{NH} 4 \mathrm{Cl} / \mathrm{NH} 4 \mathrm{OH} \mathrm{pH}=8$ solution. The reaction was extracted with $\mathrm{Et}_{2} \mathrm{O}(10$ $\mathrm{mL} \mathrm{X}$ 3), the combined organic layers were washed with brine, dried over $\mathrm{MgSO}$, filtered, concentrated under reduced pressure, and the residue was purified by column chromatography on silica gel (hexanes : ethyl acetate $10: 1$ ) to give $33 \mathrm{mg}$ of the moncyclic product 19a in $77 \%$ yield. The enantiomeric ratio was determined to be $52: 48$ from the ketal derivative of the product by NMR using $(+)-\mathrm{Eu}(\mathrm{hfc})_{3}$ as a shift reagent.

\section{Scheme 8 (production of 20)}

A solution of bis- $(S)$-oxazoline $23(61 \mathrm{mg}, 0.23 \mathrm{mmol})$ in $4 \mathrm{~mL}$ THF was added to a solution of $\mathrm{Ni}(\mathrm{COD}) 2(63 \mathrm{mg}, 0.23 \mathrm{mmol})$ in $4 \mathrm{~mL} \mathrm{THF}$ at room temperature. After stirring $5 \mathrm{~min}$, the yellow solution was cooled to $0{ }^{\circ} \mathrm{C}$ in an ice bath. A $0{ }^{\circ} \mathrm{C}$ solution of substrate $13 \mathbf{a}(41 \mathrm{mg}, 0.21 \mathrm{mmol})$ in 4 $\mathrm{mL}$ THF was added dropwise, and the resulting dark red solution was stirred for $3.5 \mathrm{~h}$ at $0{ }^{\circ} \mathrm{C}$. Then 1 $\mathrm{mL} \mathrm{MeOH}$ was added, and after $0.5 \mathrm{~h}$, the reaction was quenched by adding $3 \mathrm{~mL} \mathrm{NaHCO} 3$ sat. 
solution. The reaction mixture was extracted with Et2O (15 mL X 3), the combined organic layers were washed with brine, dried over $\mathrm{MgSO} 4$, filtered, concentrated, and the residue was purified by column chromatography on silica gel (hexanes: ethyl acetate $9: 1$ ) to give $13.7 \mathrm{mg}$ of the bicyclic compound 20 in a 33\% yield. The enantiomeric ratio was determined to be $72: 28$ by NMR using (+)$\mathrm{Eu}(\mathrm{hfc}) 3$ as a shift reagent, which was confirmed by HPLC analysis on a Chiralcel OB column eluting with hexane : 2-propanol (97:3). 
Scheme 3

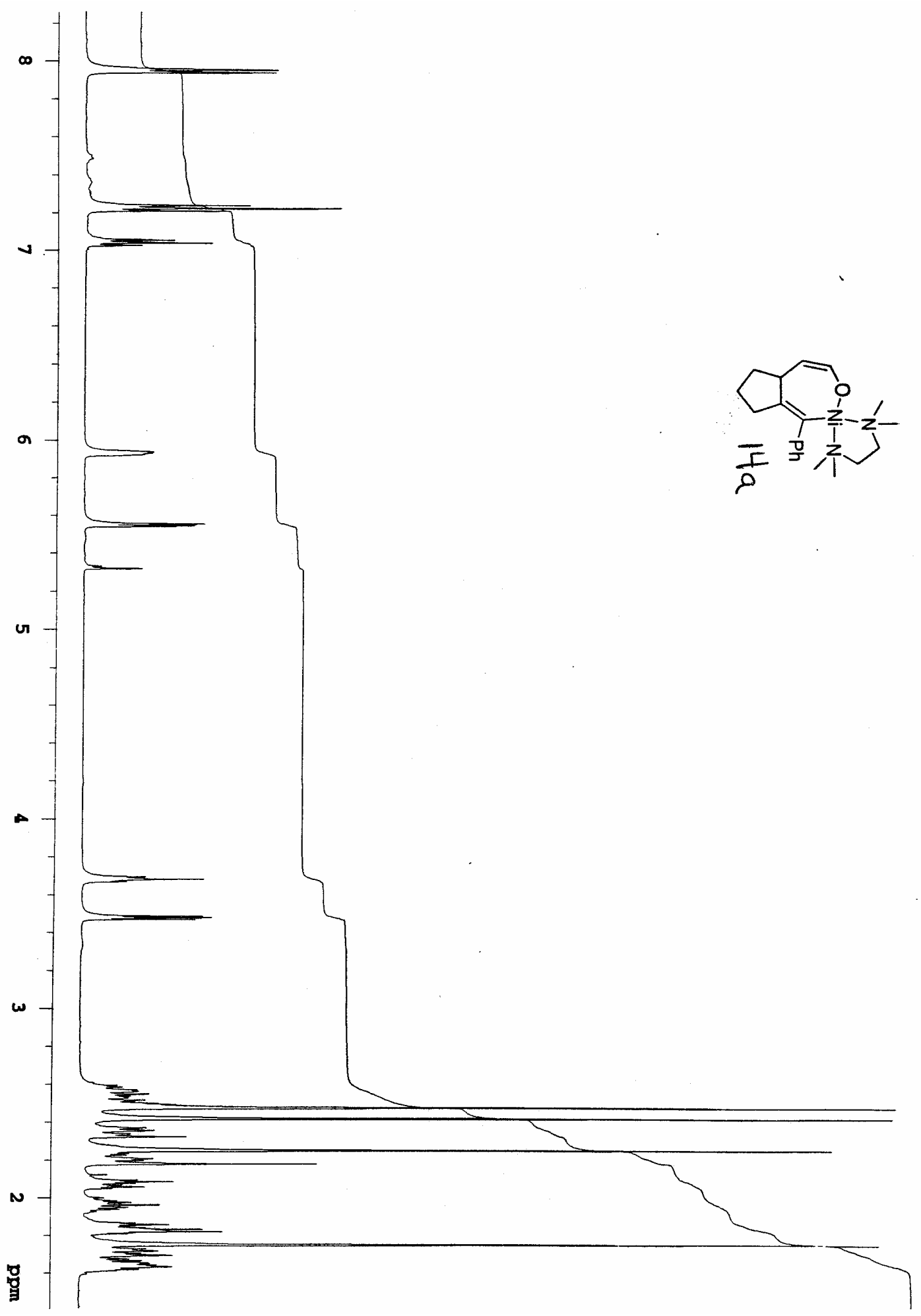




\section{Scheme 3}

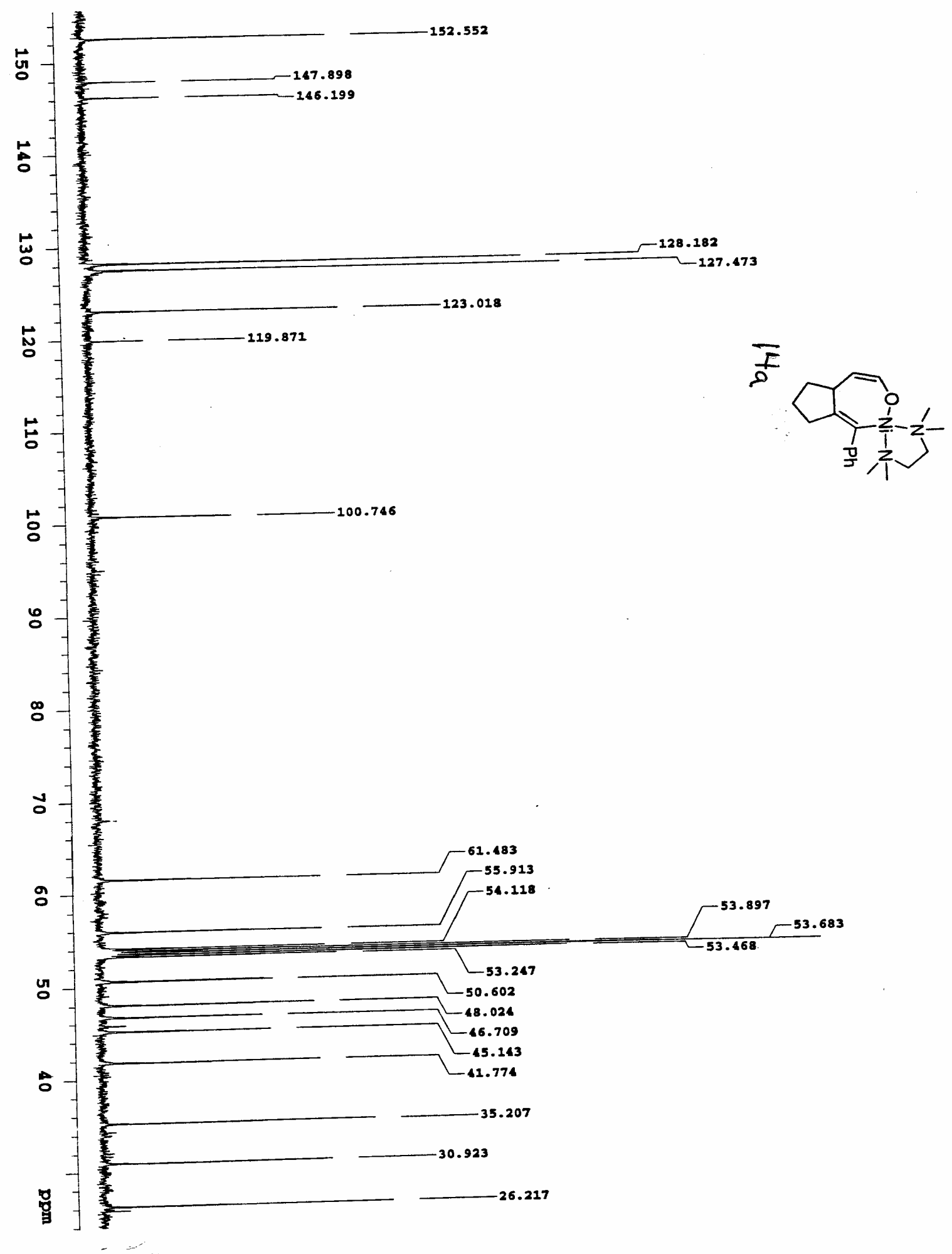


Scheme 3

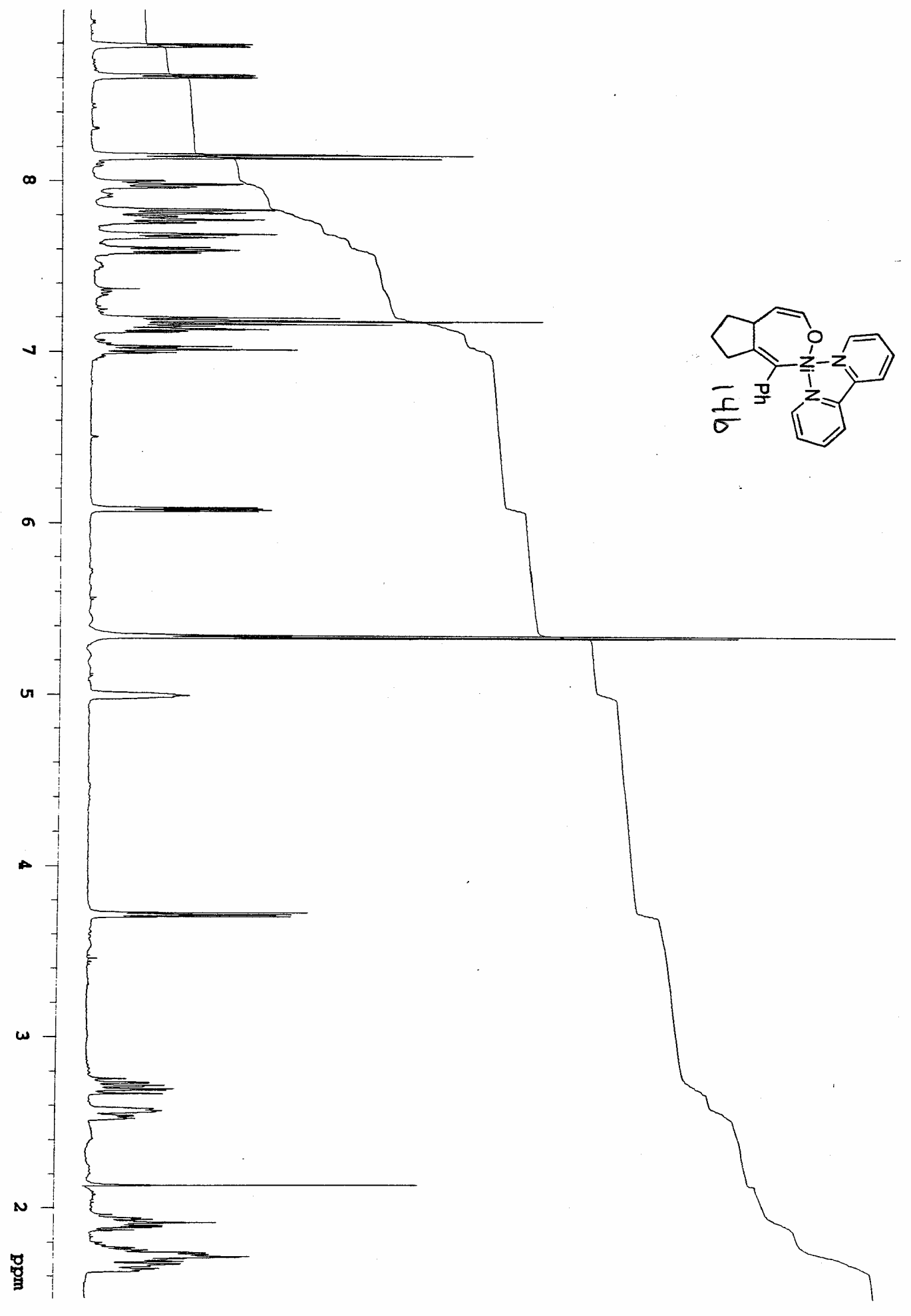




\section{Scheme 3}

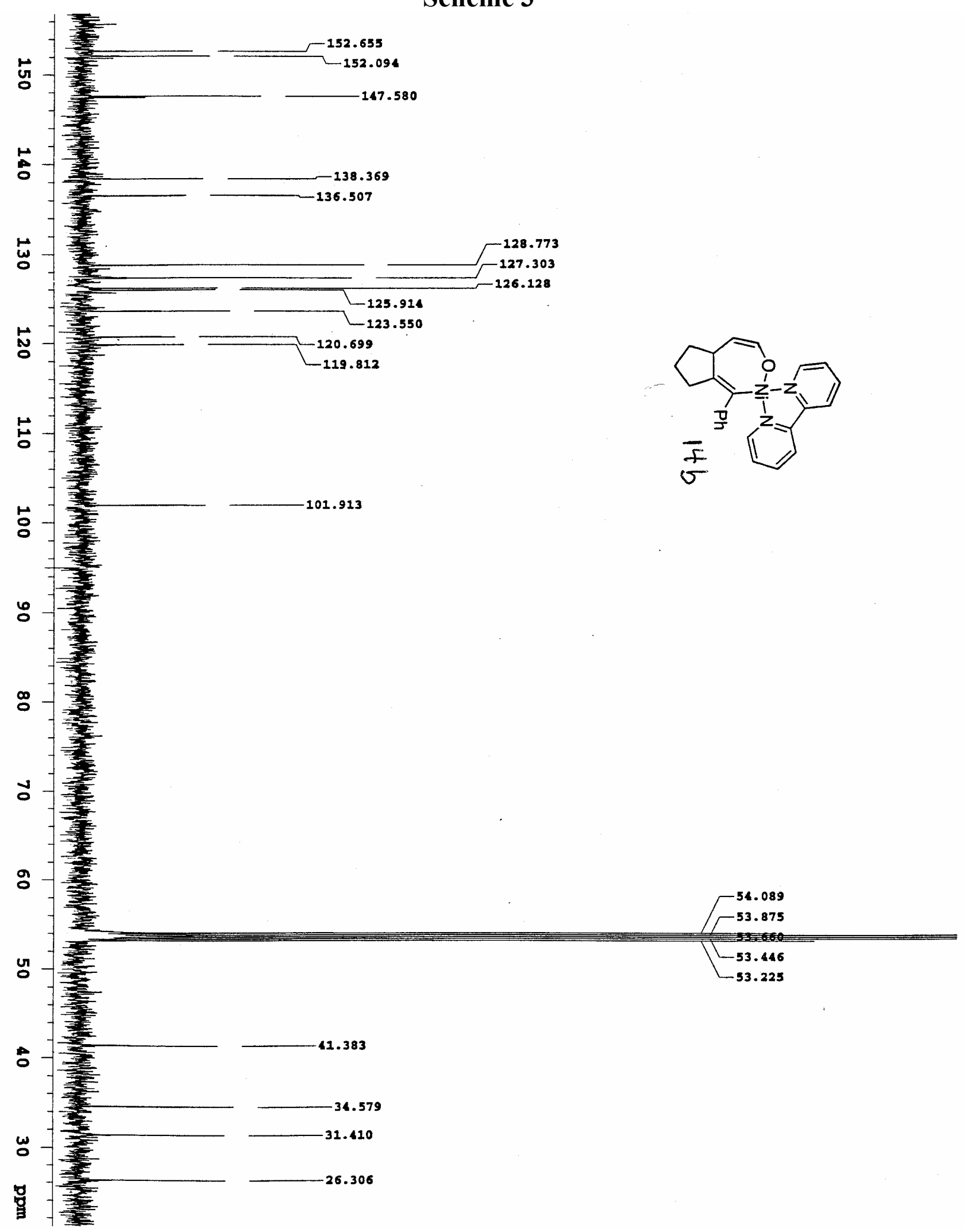




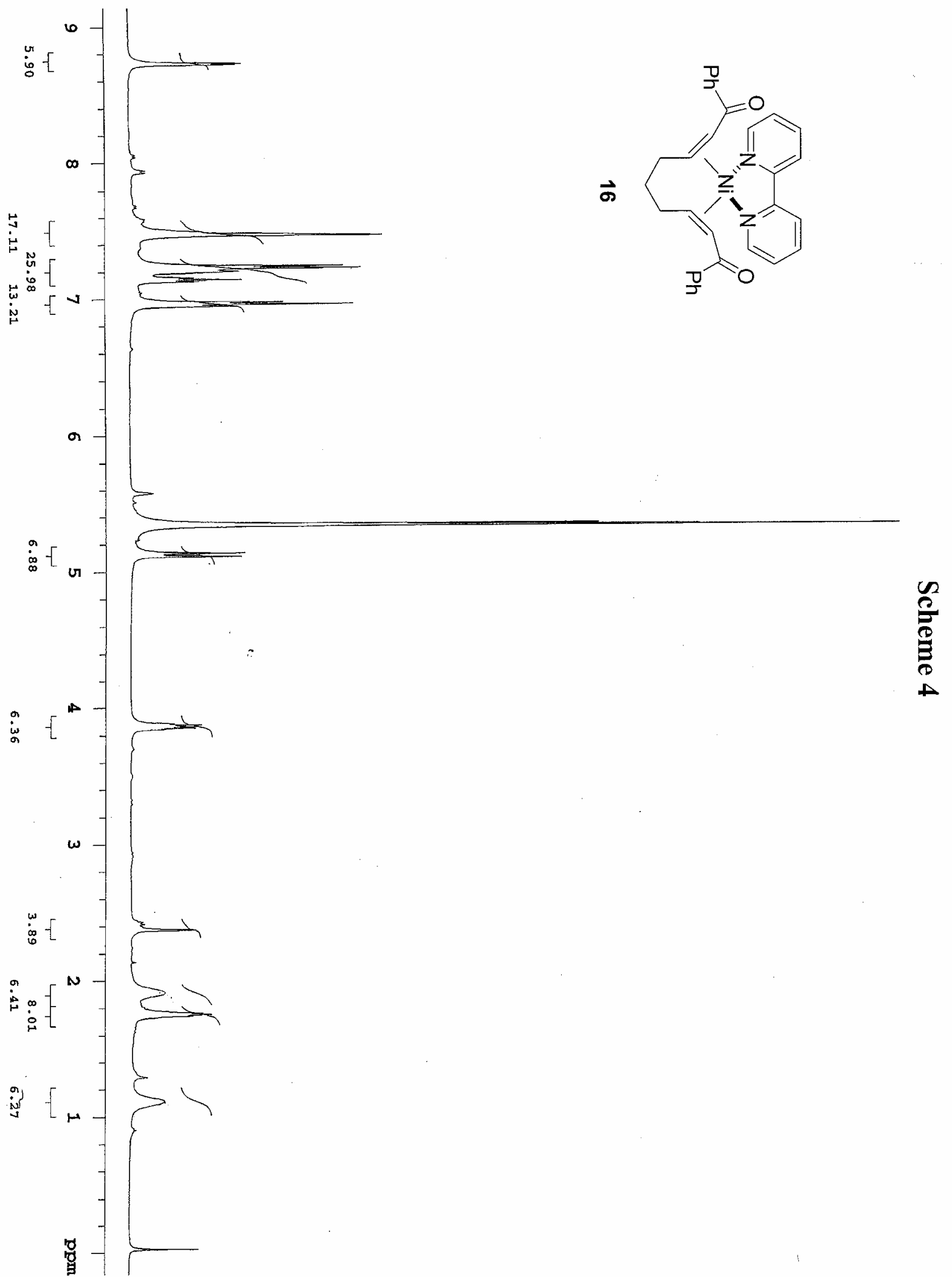


S14

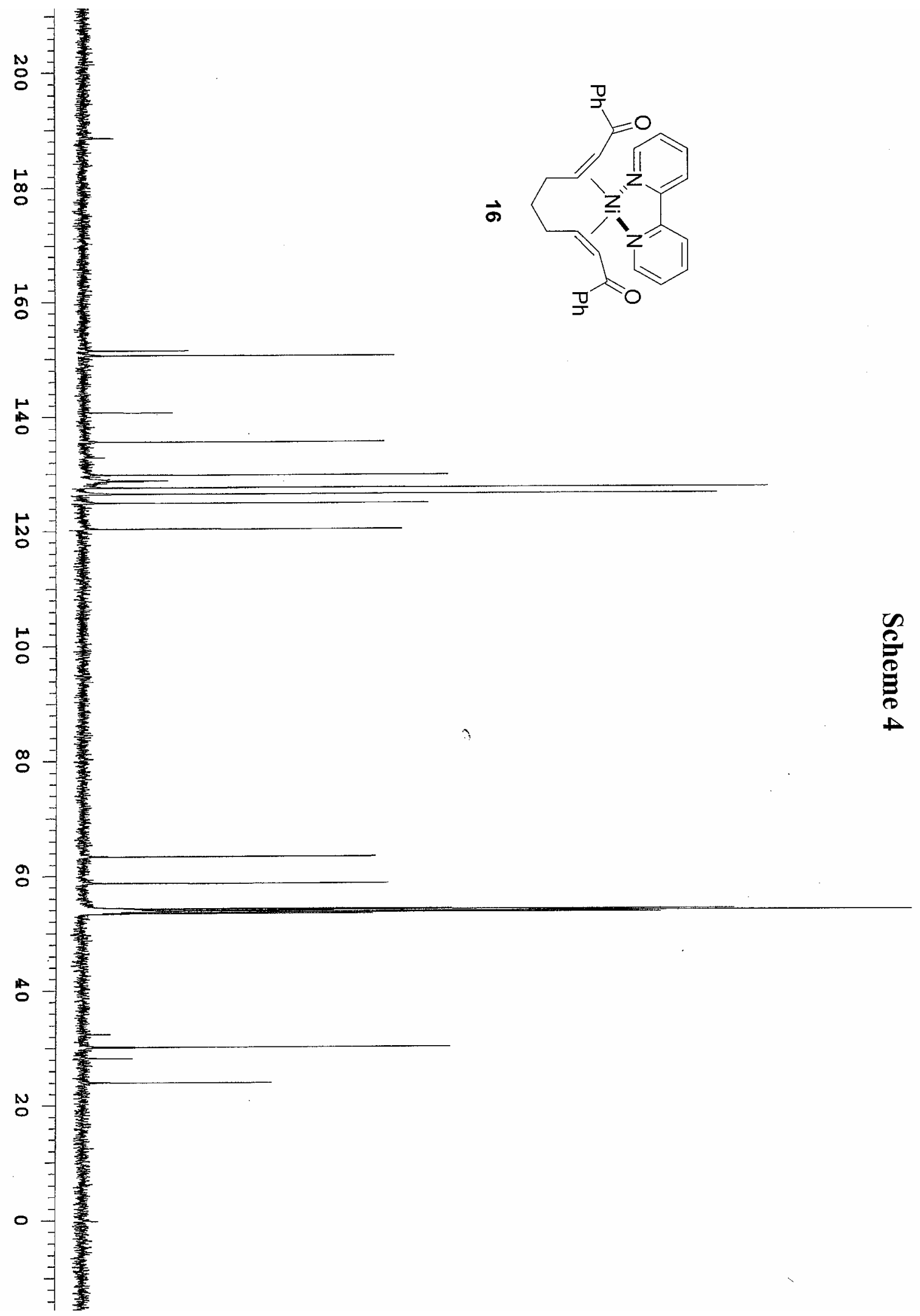


Scheme 6

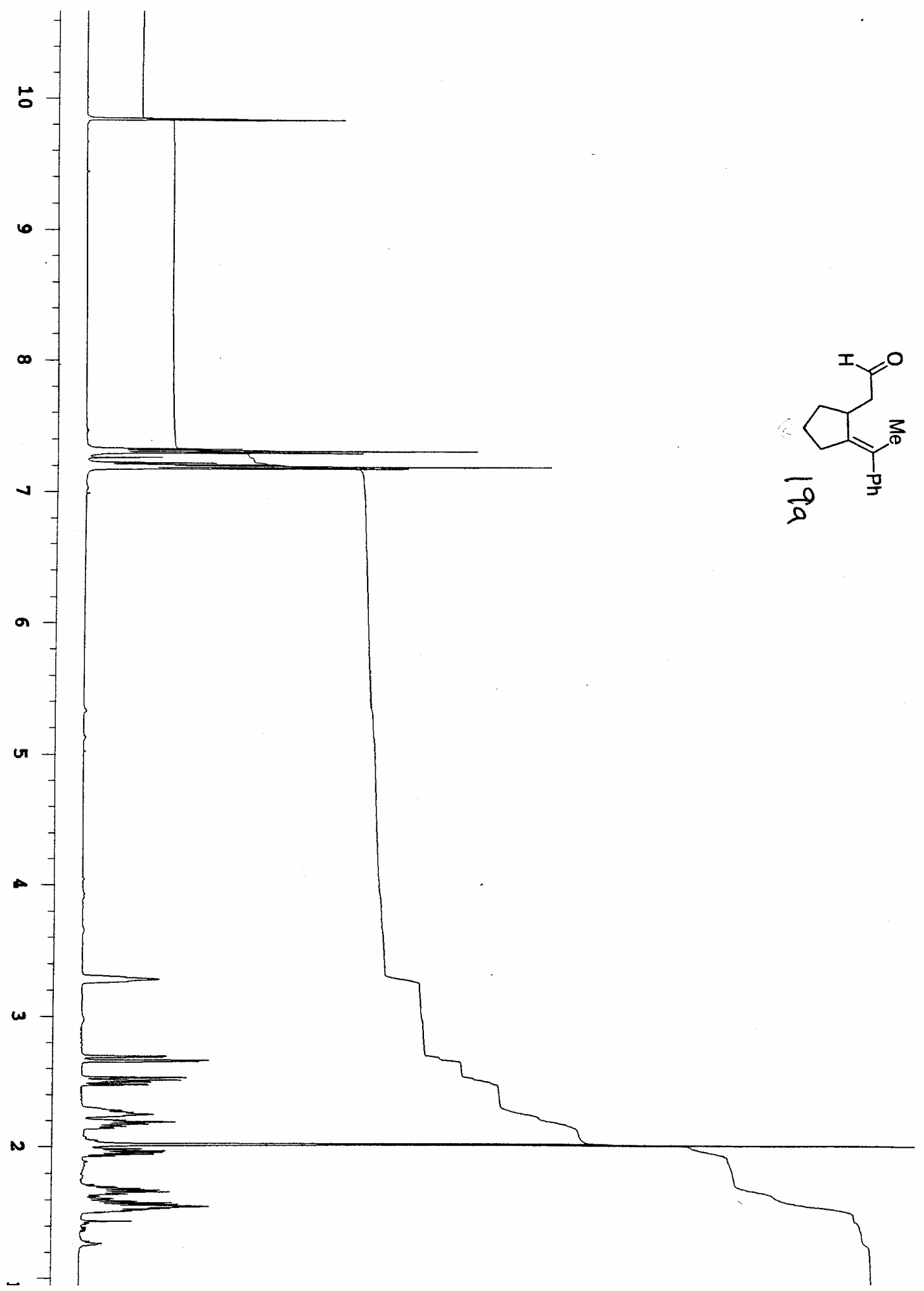




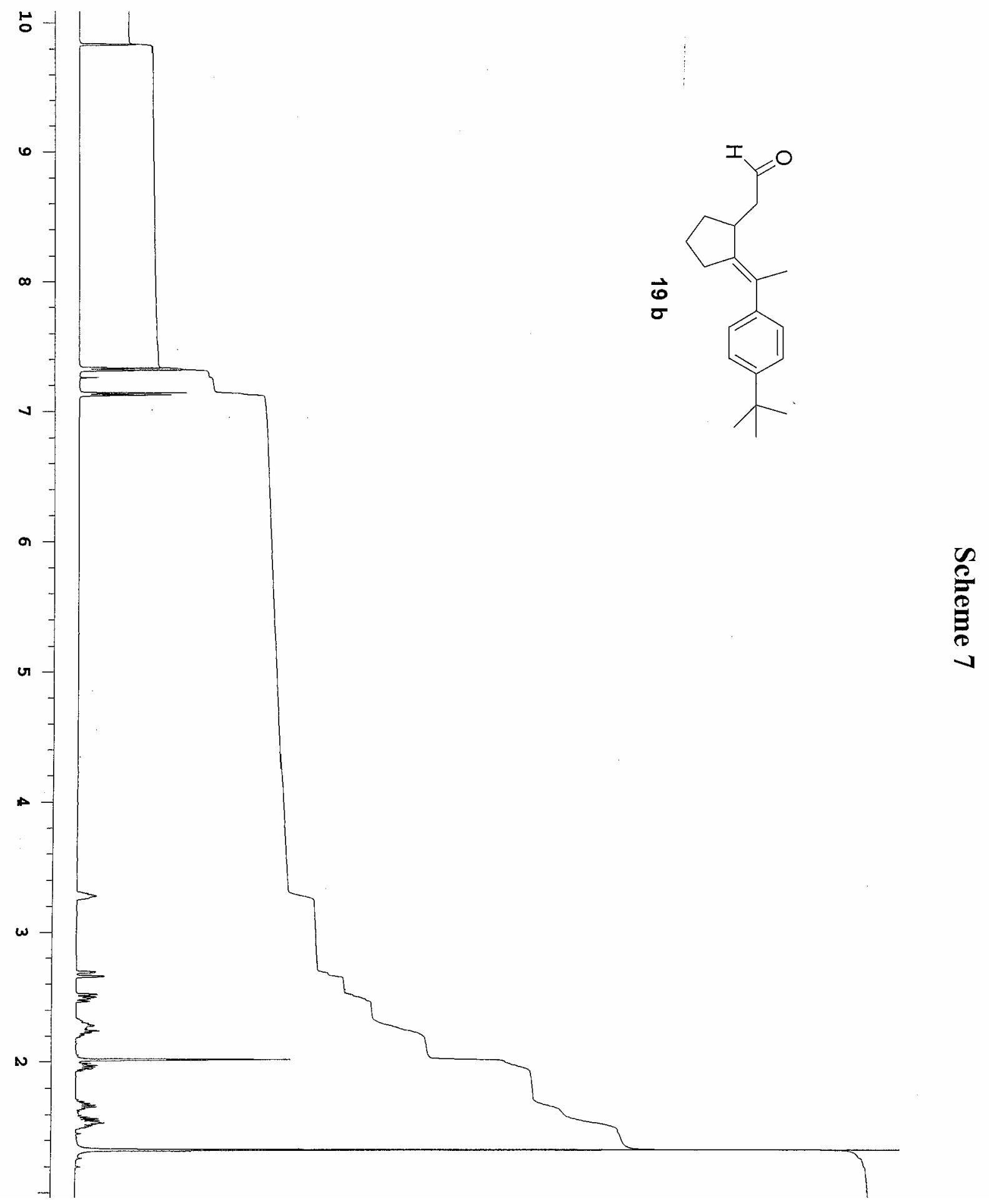




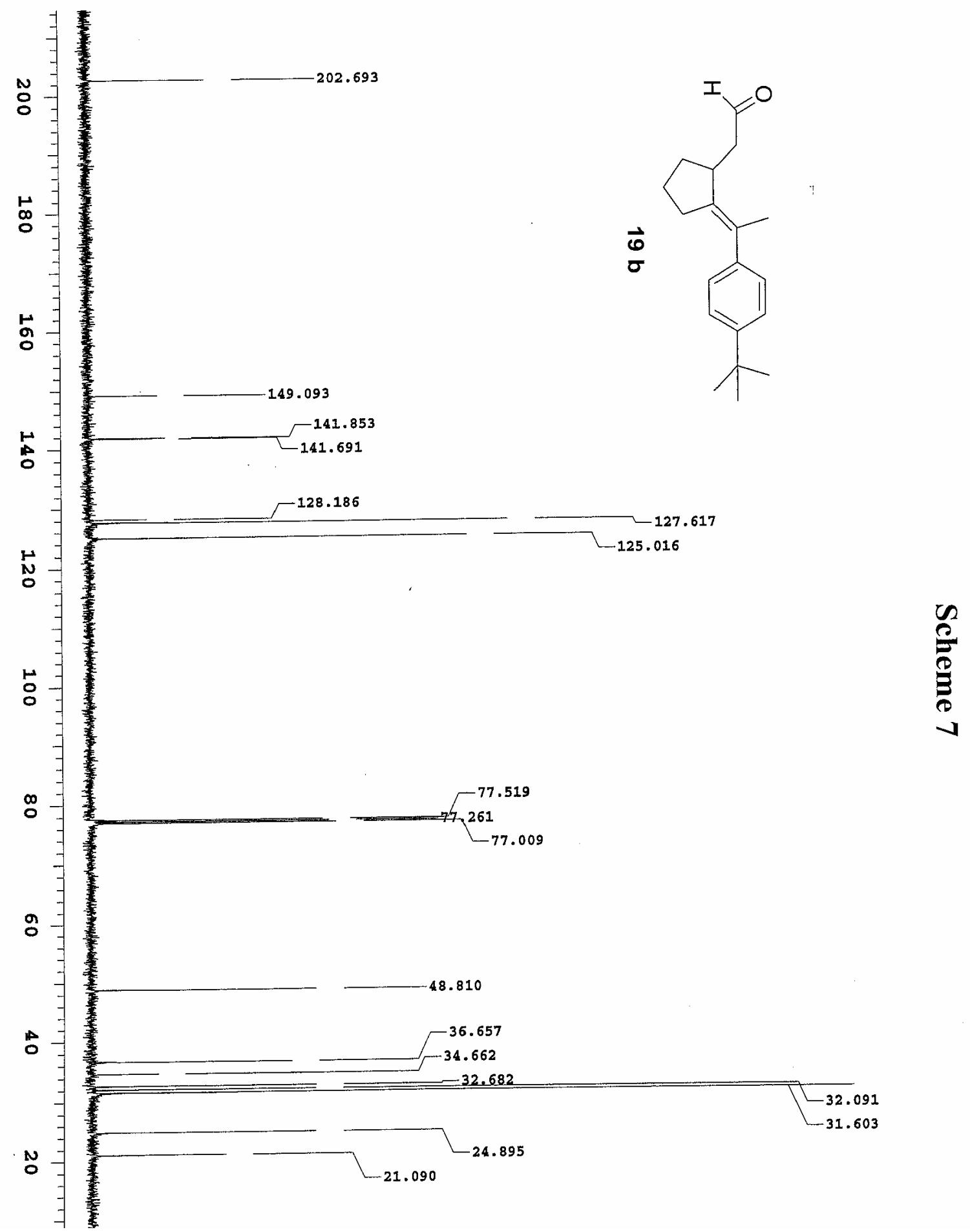


Scheme 7
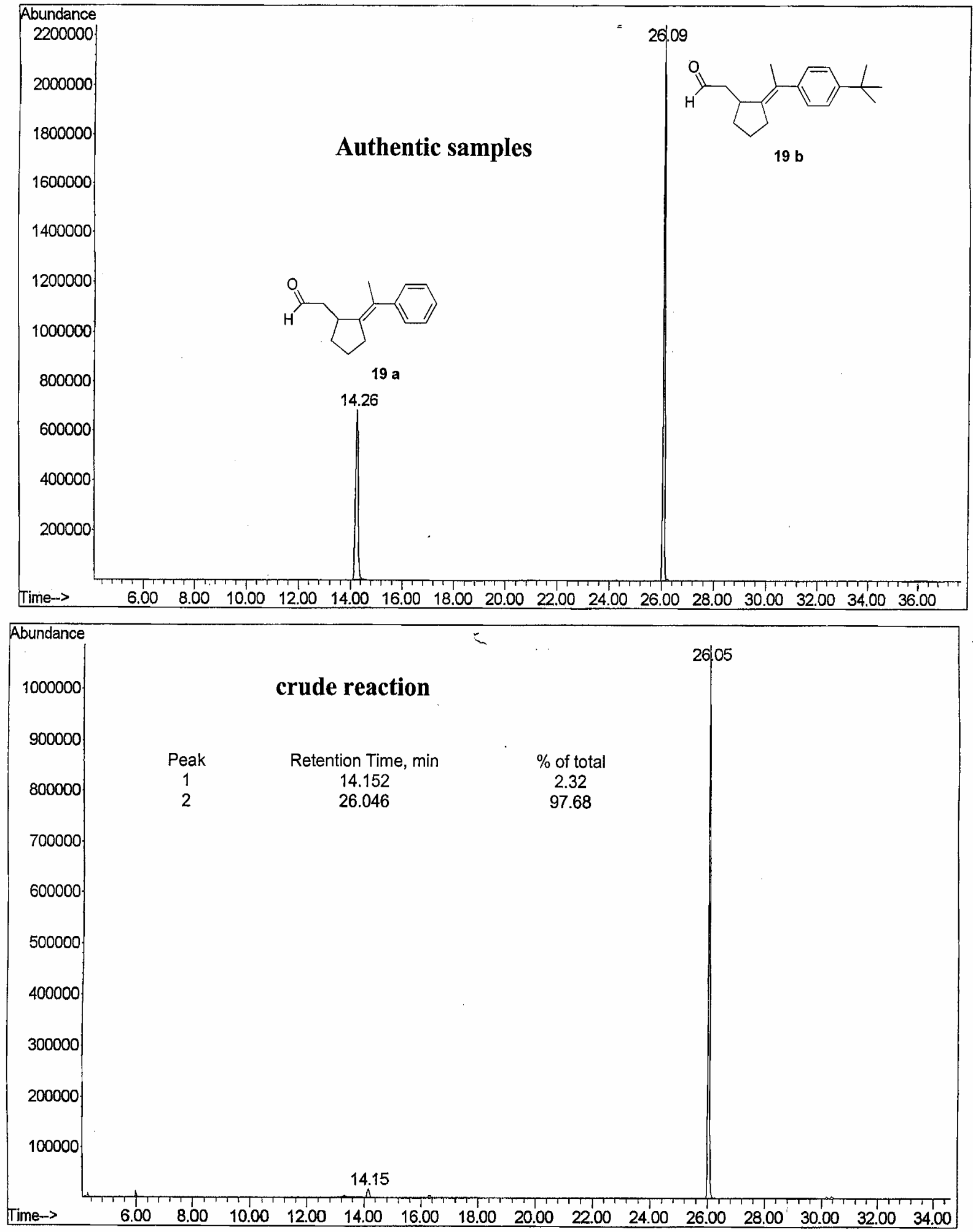


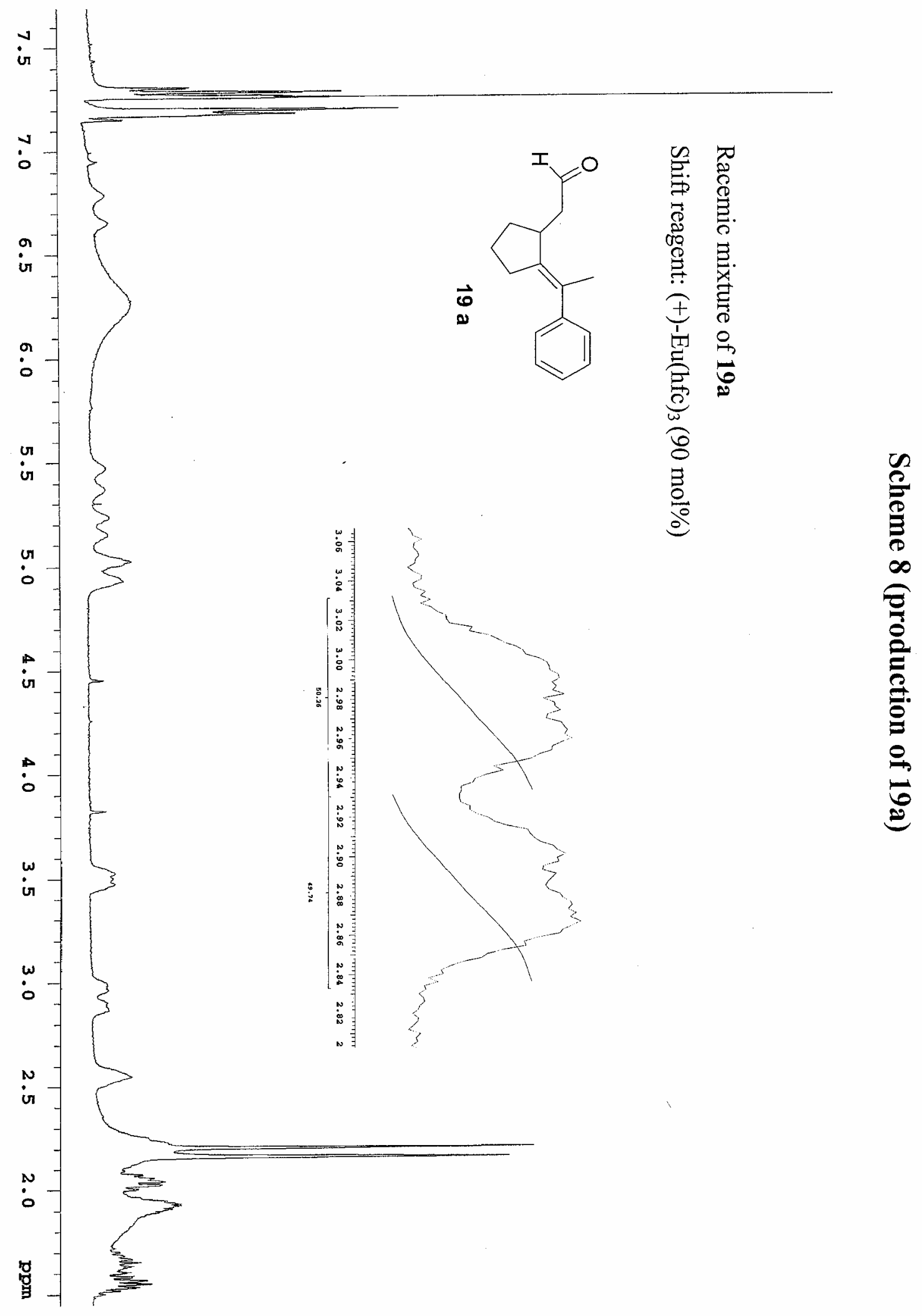




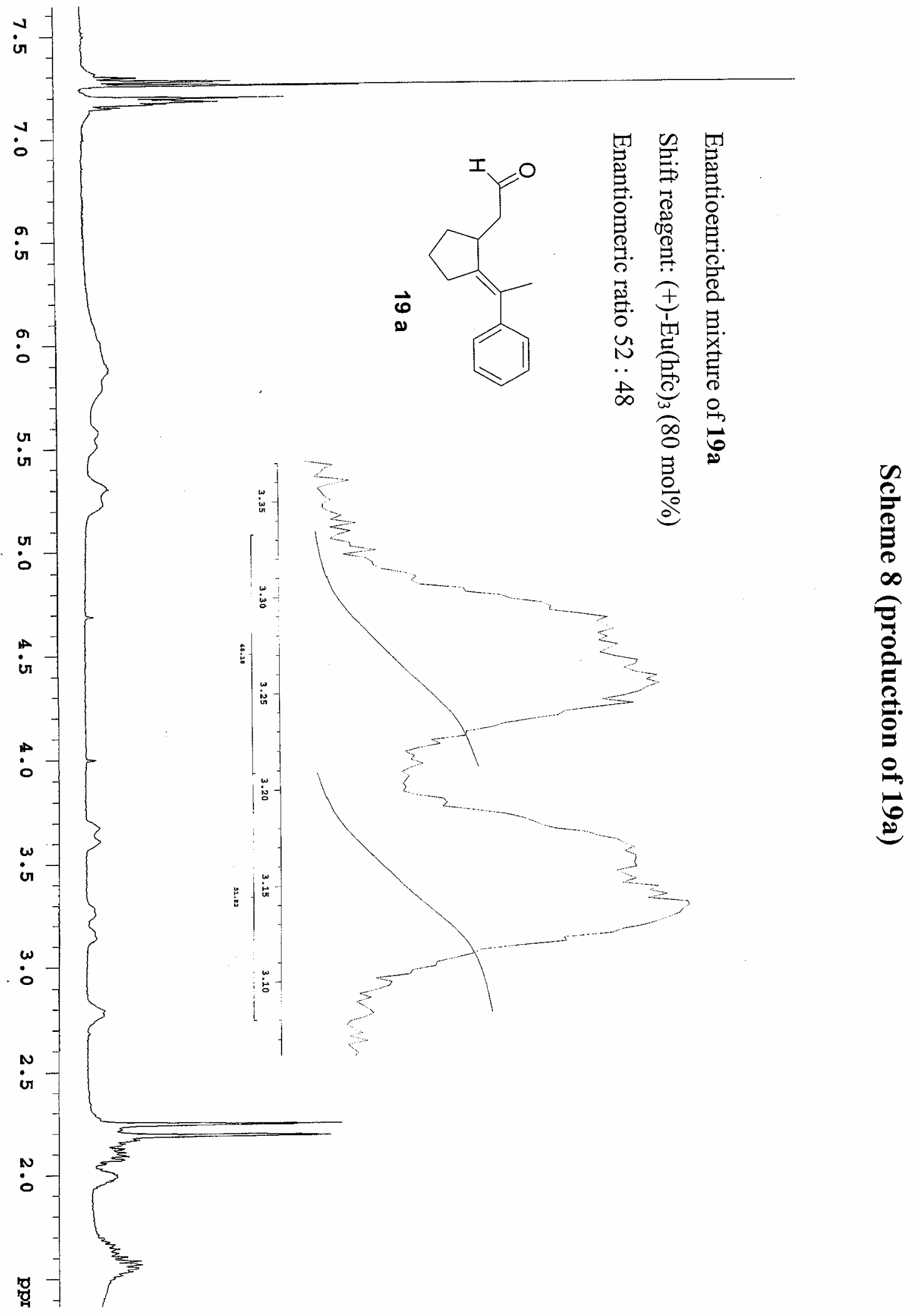



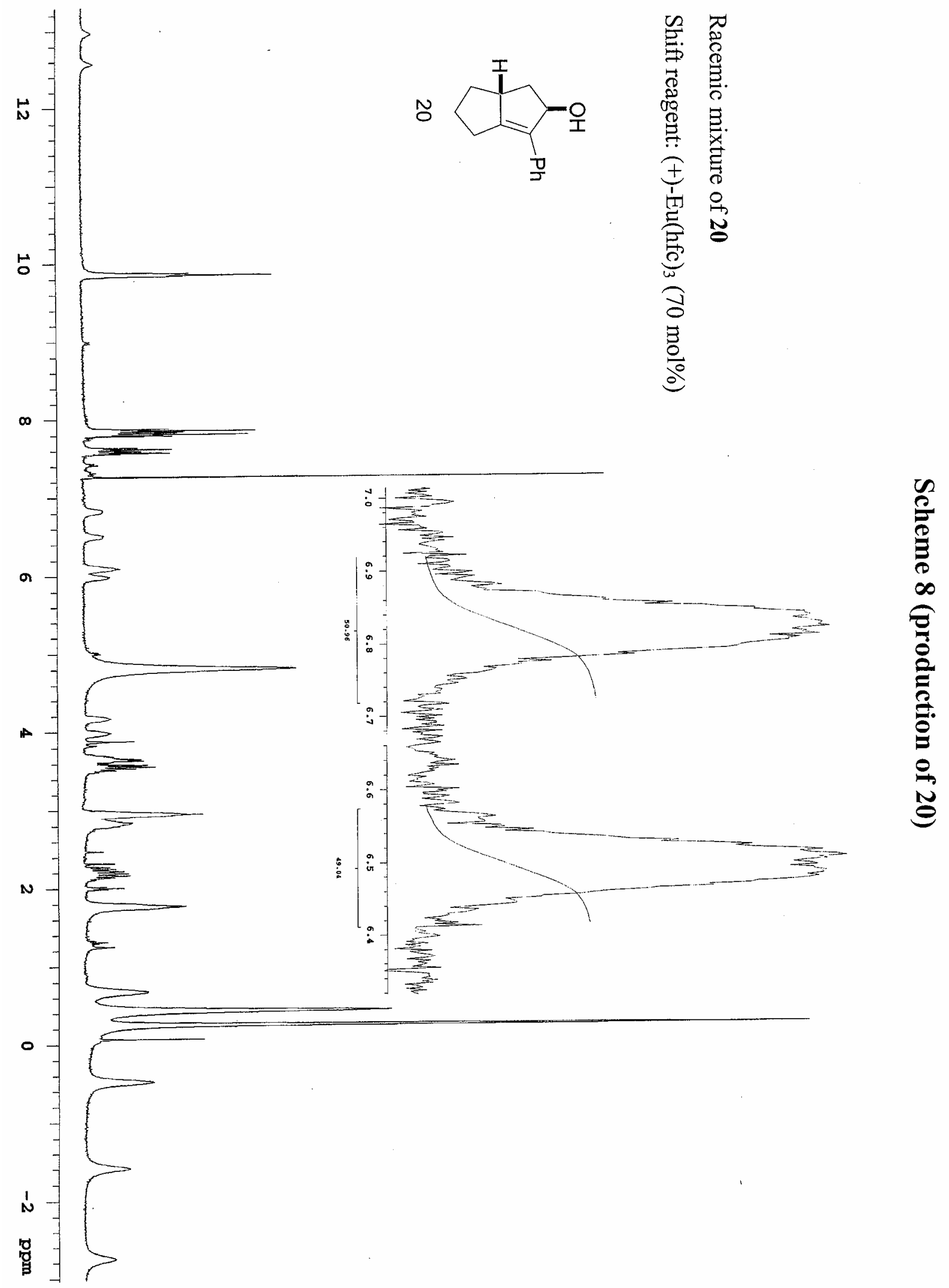


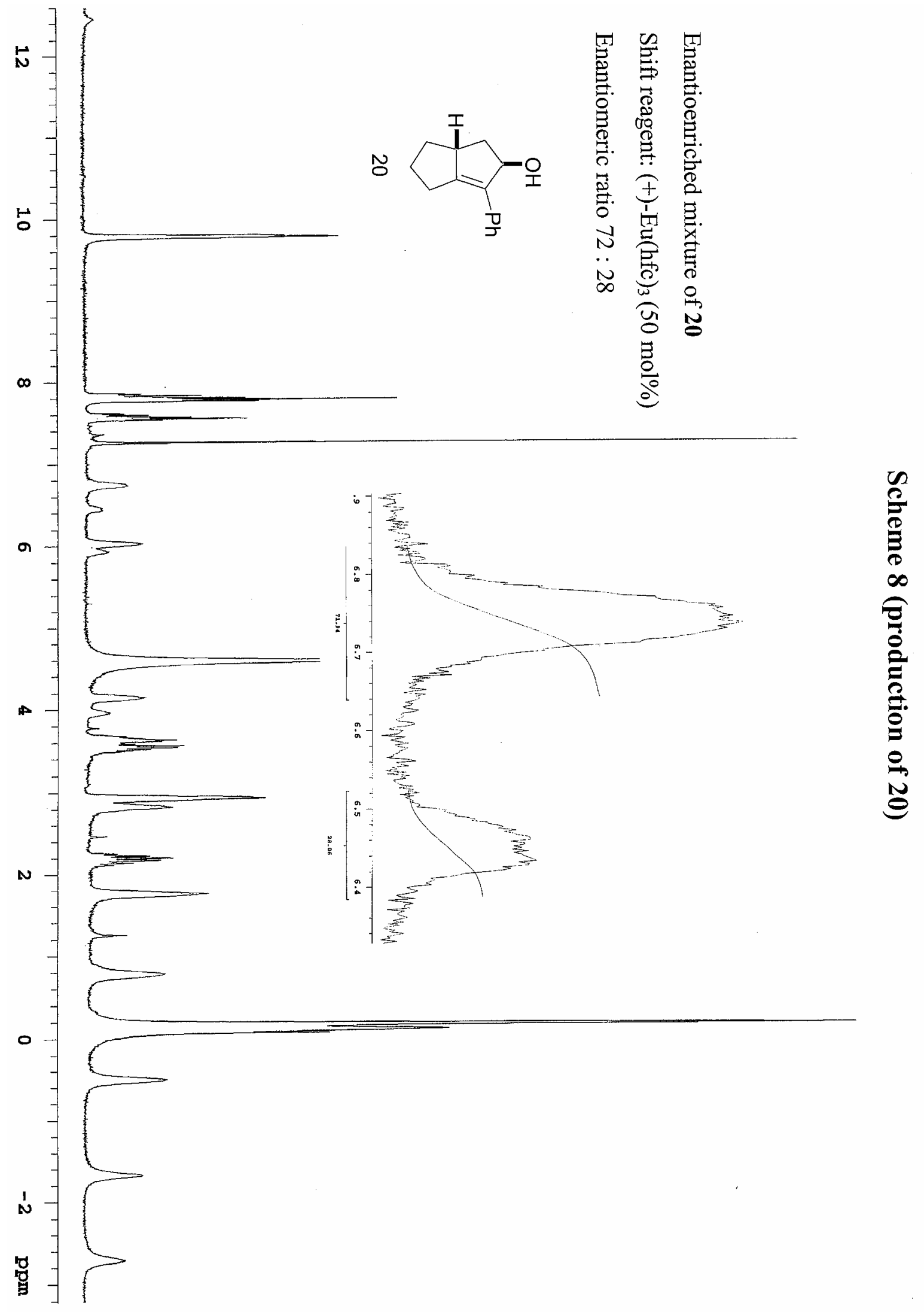




\section{Scheme 8 (production of 20)}

HPLC, Chiralcel OB column

Hexane : 2-Propanol (97: 3)

Enantiomeric ratio $74: 26$
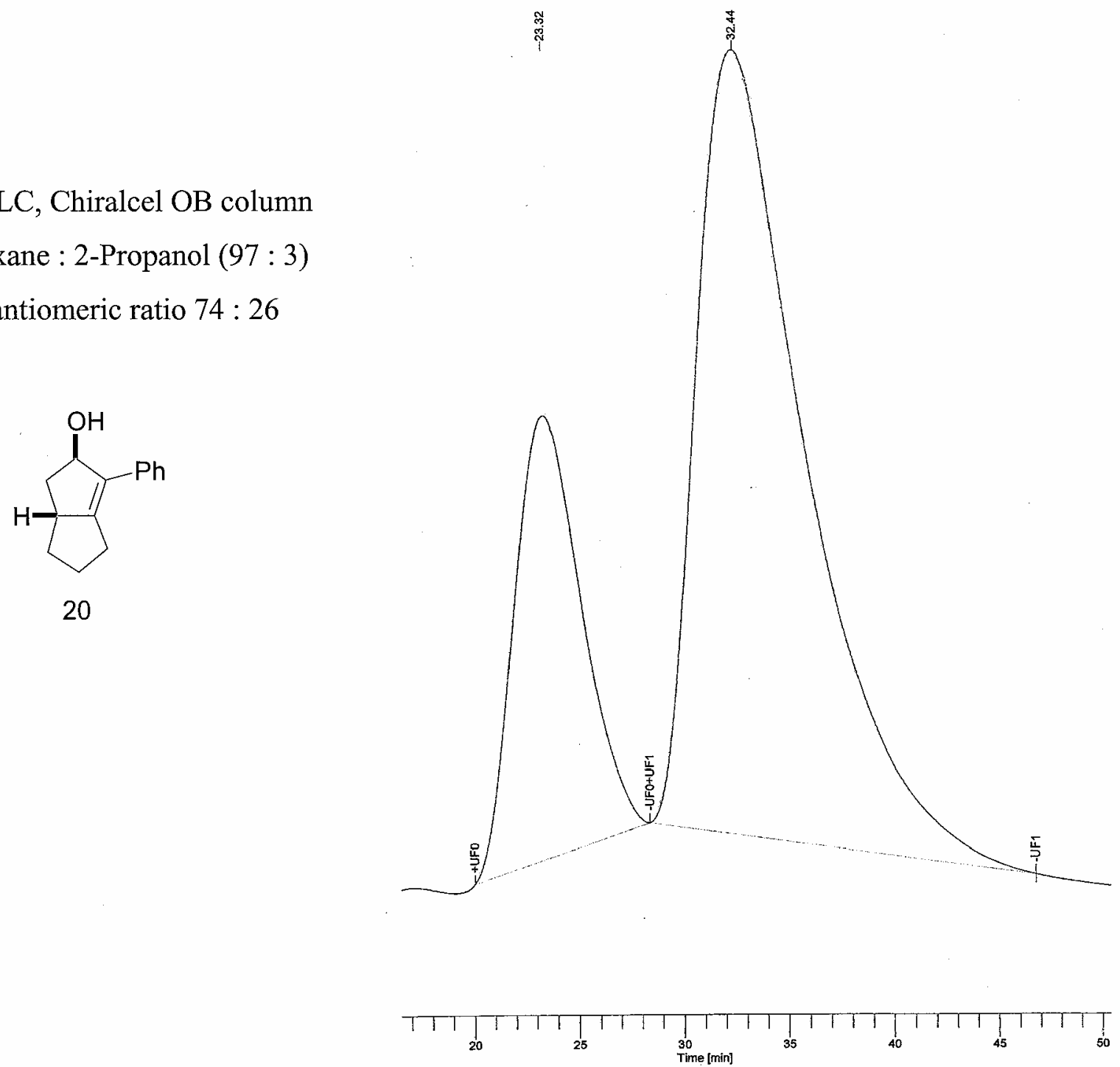
Full details for the crystallographic data of compounds $\mathbf{1 4 a}$ and $\mathbf{1 4 b}$ are available in the supporting information of reference 9a. The description of crystallographic data collection and structural analysis for compounds $\mathbf{1 6}$ and $\mathbf{1 8}$ are given on the following pages.

In structure 16, there are two independent nickel complexes in an asymmetric unit. Methylene chloride was disordered. In structure 18, there is disorder in the propylene linkage. C21 occupies two distinct positions. Only one position is shown in the diagram for clarity. 
structure 16

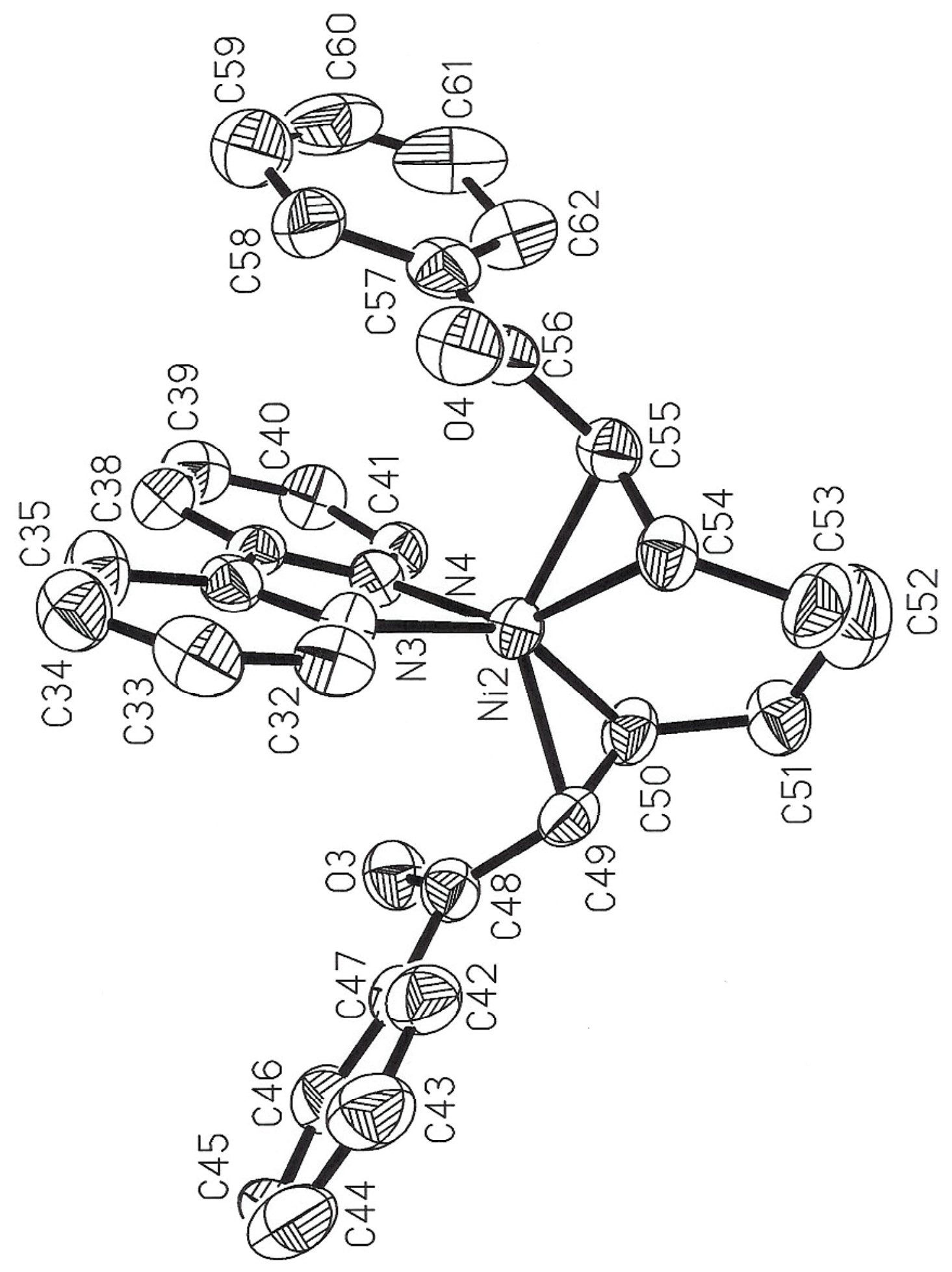


Structure 16

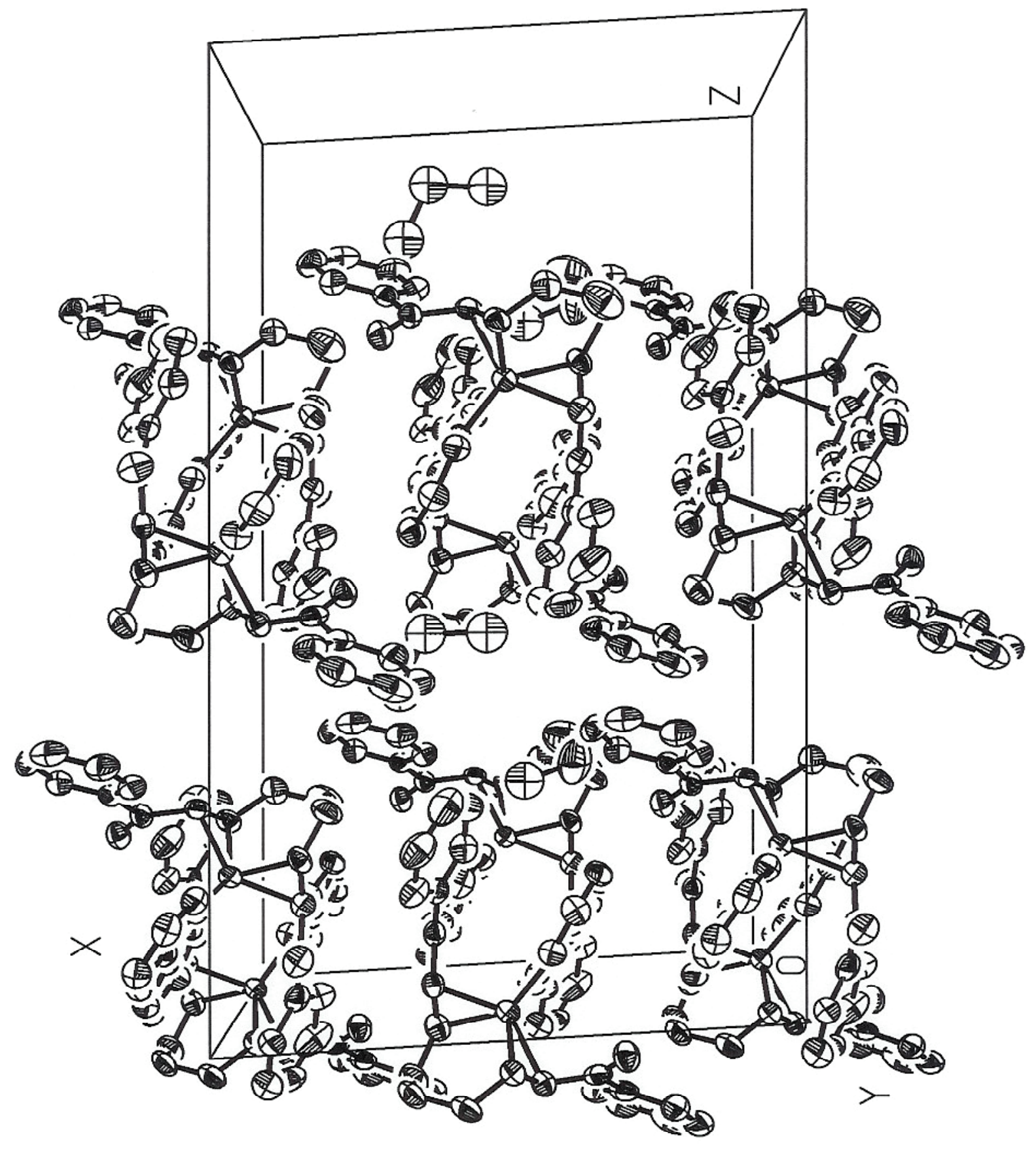


Table 1. Crystal data and structure refinement for 16

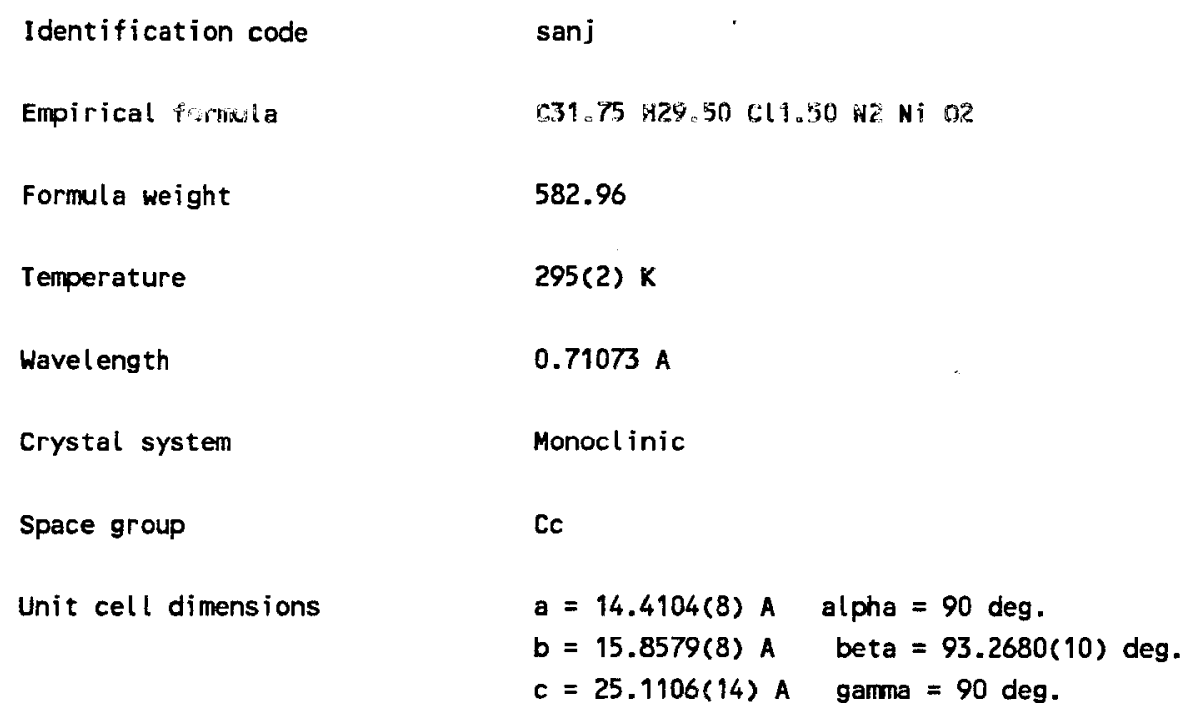


Table 2. Atomic coordinates $(\times 10 \times 4)$ and equivalent isotropic displacement parameters $\left(A^{\wedge} 2 \times 10^{\wedge} 3\right.$ ) for $1 . \quad U(e q)$ is defined as one third of the trace of the orthogonalized $U i j$ tensor.

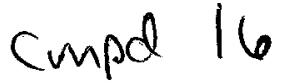

\begin{tabular}{|c|c|c|c|c|}
\hline & $x$ & $y$ & z & $U(e q)$ \\
\hline $\mathrm{Ni}(1)$ & $1(1)$ & $1807(1)$ & $10002(1)$ & $36(1)$ \\
\hline $\mathrm{Ni}(2)$ & $44(1)$ & $3810(1)$ & $16574(1)$ & $35(9)$ \\
\hline$N(1)$ & $-553(3)$ & $761(3)$ & $10314(2)$ & $39(1)$ \\
\hline$N(2)$ & $-750(3)$ & $2333(3)$ & $10571(2)$ & $36(1)$ \\
\hline$c(1)$ & $-460(5)$ & $-34(4)$ & $10143(3)$ & $46(2)$ \\
\hline$c(2)$ & $-864(5)$ & $-709(4)$ & $10376(3)$ & $56(2)$ \\
\hline$C(3)$ & $-1377(5)$ & $-578(4)$ & $10812(3)$ & $55(2)$ \\
\hline$c(4)$ & $-1496(5)$ & $240(4)$ & $10993(3)$ & $46(2)$ \\
\hline$C(5)$ & $-1066(4)$ & $894(4)$ & $10731(2)$ & $35(1)$ \\
\hline$c(6)$ & $-1161(4)$ & $1778(3)$ & $10892(3)$ & $33(1)$ \\
\hline$c(7)$ & $-1583(5)$ & $2024(4)$ & $11350(3)$ & $45(2)$ \\
\hline$c(8)$ & $-1595(5)$ & $2882(4)$ & $11479(3)$ & $50(2)$ \\
\hline$c(9)$ & $-1177(5)$ & $3452(4)$ & $11147(3)$ & $53(2)$ \\
\hline$c(10)$ & $-776(5)$ & $3149(4)$ & $10705(3)$ & $42(2)$ \\
\hline$c(11)$ & $1643(5)$ & $2723(5)$ & $11380(3)$ & $56(2)$ \\
\hline$c(12)$ & $1561(8)$ & $3177(7)$ & $11838(5)$ & $80(3)$ \\
\hline$c(13)$ & $1088(8)$ & $2848(8)$ & $12253(5)$ & $94(3)$ \\
\hline$c(14)$ & $724(7)$ & $2093(8)$ & $12206(4)$ & $88(3)$ \\
\hline$C(15)$ & $795(6)$ & $1614(6)$ & $11753(3)$ & $66(2)$ \\
\hline$c(16)$ & $1252(4)$ & $1927(4)$ & $11334(3)$ & $45(2)$ \\
\hline$c(17)$ & $1328(4)$ & $1384(4)$ & $10847(3)$ & $41(2)$ \\
\hline$O(1)$ & $1312(4)$ & $615(3)$ & $10891(2)$ & $56(1)$ \\
\hline$c(18)$ & $1340(5)$ & $1815(4)$ & $10334(3)$ & $39(2)$ \\
\hline$c(19)$ & $1273(5)$ & $1397(5)$ & $9843(3)$ & $47(2)$ \\
\hline$c(20)$ & $1694(6)$ & $1705(5)$ & $9348(3)$ & $60(2)$ \\
\hline$c(21)$ & $1690(5)$ & $2658(5)$ & $9281(3)$ & $60(2)$ \\
\hline$c(22)$ & $729(6)$ & $3041(5)$ & $9140(3)$ & $59(2)$ \\
\hline$c(23)$ & $-85(4)$ & $2715(4)$ & $9451(2)$ & $40(1)$ \\
\hline$c(24)$ & $-636(4)$ & $2019(4)$ & $9250\langle 3\rangle$ & $42(1)$ \\
\hline$c(25)$ & $-1607(5)$ & $1961(4)$ & $9302(3)$ & $45(2)$ \\
\hline$O(2)$ & $-2098(3)$ & $2505(3)$ & $9529(2)$ & $56(1)$ \\
\hline$c(26)$ & $-2099(5)$ & $1234(4)$ & $9043\langle 3\rangle$ & $51(2)$ \\
\hline$c(27)$ & $-3027(6)$ & $1311(6)$ & $8839(3)$ & $60(2)$ \\
\hline$c(28)$ & $-3485(7)$ & $654(7)$ & $8581(3)$ & $73(2)$ \\
\hline$C(29)$ & $-3029(8)$ & $-113(7)$ & $8548(4)$ & $86(4)$ \\
\hline$c(30)$ & $-2140(7)$ & $-215(6)$ & $8748(3)$ & $70(2)$ \\
\hline$c(31)$ & $-1655(6)$ & $441(5)$ & $8985(3)$ & $58(2)$ \\
\hline$N(3)$ & $581(3)$ & $2747(3)$ & $16278(2)$ & $38(1)$ \\
\hline$N(4)$ & $804(3)$ & $4308(3)$ & $16007(2)$ & $35(1)$ \\
\hline$c(32)$ & $476(5)$ & $1951(4)$ & $16449(3)$ & $48(2)$ \\
\hline$c(33)$ & $879(5)$ & $1268(4)$ & $16214(3)$ & $54(2)$ \\
\hline$c(34)$ & $1410(5)$ & $1385(4)$ & $15778(3)$ & $52(2)$ \\
\hline$c(35)$ & $1512(5)$ & $2192(4)$ & $15588(3)$ & $53(2)$ \\
\hline$c(36)$ & $1096(4)$ & $2862(4)$ & $15849(2)$ & $38(1)$ \\
\hline$c(37)$ & $1174(4)$ & $3746(4)$ & $15680(3)$ & $36(1)$ \\
\hline$c(38)$ & $1599(5)$ & $3995(4)$ & $15220(3)$ & $46(2)$ \\
\hline$c(39)$ & $1615(5)$ & $4825(5)$ & $15076(3)$ & $53(2)$ \\
\hline$C(40)$ & $1222(5)$ & $5407(4)$ & $15405(3)$ & $47(2)$ \\
\hline $\begin{array}{l}c(41) \\
c(42)\end{array}$ & $\begin{array}{r}859(5) \\
1626(6)\end{array}$ & $\begin{array}{l}5137(4) \\
2415(5)\end{array}$ & $\begin{array}{l}15870(3) \\
17639(3)\end{array}$ & $\begin{array}{l}42(1) \\
52(2)\end{array}$ \\
\hline
\end{tabular}




\begin{tabular}{|c|c|c|c|c|}
\hline$c(43)$ & $2047(6)$ & $1711(5)$ & $17889(3)$ & $62(2)$ \\
\hline$c(44)$ & $2974(7)$ & $1744(5)$ & $18059(3)$ & $64(2)$ \\
\hline$c(45)$ & $3455(7)$ & $2477(6)$ & $17968(3)$ & $69(3)$ \\
\hline$c(46)$ & $3033(5)$ & $3173(5)$ & $17705(3)$ & $55(2)$ \\
\hline$c(47)$ & $2115(5)$ & $3147(4)$ & $17540(3)$ & $42(2)$ \\
\hline$c(48)$ & $1666(4)$ & $3918(4)$ & $17275(3)$ & $41(2)$ \\
\hline$O(3)$ & $2156(3)$ & $4425(3)$ & $17059(2)$ & $50(1)$ \\
\hline$c(49)$ & $661(5)$ & $4004(4)$ & $17304(2)$ & $40(2)$ \\
\hline$c(50)$ & $168(4)$ & $4722(4)$ & $17126(2)$ & $44(2)$ \\
\hline$c(51)$ & $-657(5)$ & $5059(4)$ & $17417(3)$ & $53(2)$ \\
\hline$c(52)$ & $-1613(7)$ & $4749(6)$ & $17263(4)$ & $94(3)$ \\
\hline$c(53)$ & $-1709(6)$ & $3817(5)$ & $17203(3)$ & $64(2)$ \\
\hline$c(54)$ & $-1249(4)$ & $3429(4)$ & $16727(3)$ & $43(2)$ \\
\hline$c(55)$ & $-1277(5)$ & $3823(4)$ & $16224(3)$ & $41(2)$ \\
\hline$c(56)$ & $-1249(5)$ & $3368(4)$ & $15726(3)$ & $42(2)$ \\
\hline $0(4)$ & $-1281(4)$ & $2588(3)$ & $15710(2)$ & $58(1)$ \\
\hline$c(57)$ & $-1165(5)$ & $3855(4)$ & $15220(3)$ & $48(2)$ \\
\hline$c(58)$ & $-739(5)$ & $3451(6)$ & $14798(3)$ & $65(2)$ \\
\hline$c(59)$ & $-616(7)$ & $3888(8)$ & $94313(4)$ & $85(3)$ \\
\hline$c(60)$ & $-939(7)$ & $4729(9)$ & $14272(4)$ & $91(4)$ \\
\hline$c(61)$ & $-1372(8)$ & $5095(7)$ & $14657(4)$ & $83(3)$ \\
\hline$c(62)$ & $-1496(6)$ & $4681(4)$ & $15156(3)$ & $60(2)$ \\
\hline$c(63)$ & $-6263(7)$ & $5731(7)$ & $7519(6)$ & $114(4)$ \\
\hline $\mathrm{Cl}(1)$ & $-6098(3)$ & $6739(2)$ & $7751(1)$ & $114(1)$ \\
\hline$c l(2)$ & $-5316(5)$ & $5212(4)$ & $7361(3)$ & $109(2)$ \\
\hline $\mathrm{Cl}\left(2^{\prime}\right)$ & $-5202(6)$ & $5162(5)$ & $7673(4)$ & $145(3)$ \\
\hline$c(64)$ & $1495(18)$ & $1108(15)$ & $4043(10)$ & $111(7)$ \\
\hline$c l(3)$ & $377(5)$ & $1500(4)$ & $4020(3)$ & $115(2)$ \\
\hline $\mathrm{Cl}(4)$ & $1960(11)$ & $1037(10)$ & $3436(6)$ & $120(4)$ \\
\hline $\mathrm{Cl}\left(4^{\prime}\right)$ & $1682(12)$ & $502(11)$ & $3535(7)$ & $136(5)$ \\
\hline
\end{tabular}




\section{Cimpd 16}

Table 3. Bond lengths [A] and angles [deg]

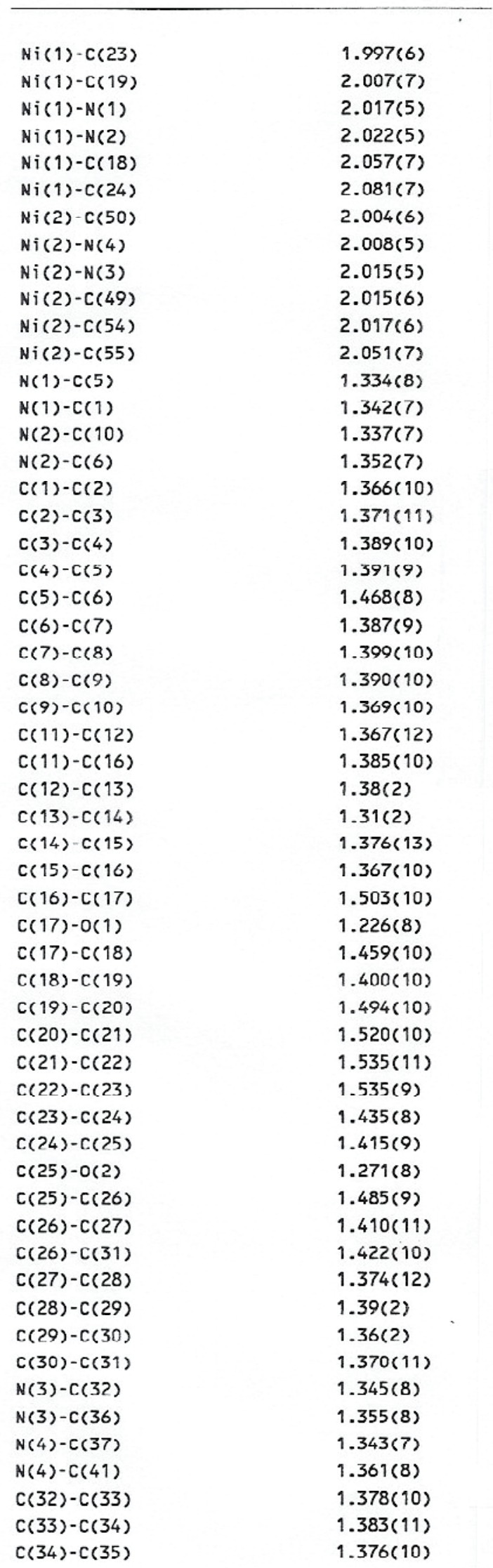

\begin{tabular}{|c|c|}
\hline$c(35)-c(36)$ & $1.401(9)$ \\
\hline$c(36)-c(37)$ & $1.471(8)$ \\
\hline$c(37)-c(38)$ & $1.395(9)$ \\
\hline$c(38)-c(39)$ & $1.365(10)$ \\
\hline$c(39)-c(40)$ & $1.381(10)$ \\
\hline$c(40)-c(41)$ & $1.379(9)$ \\
\hline$c(42)-c(47)$ & $1.388(10)$ \\
\hline$c(42)-c(43)$ & $1.402(10)$ \\
\hline$c(43)-c(44)$ & $1.379(12)$ \\
\hline$c(44)-c(45)$ & $1.379(13)$ \\
\hline$c(45)-c(46)$ & $1.407(11)$ \\
\hline$c(46)-c(47)$ & $1.364(10)$ \\
\hline$c(47)-c(48)$ & $1.519(9)$ \\
\hline$c(48)-0(3)$ & $1.218(8)$ \\
\hline$c(48)-c(49)$ & $1.461(9)$ \\
\hline$c(49)-c(50)$ & $1.402(9)$ \\
\hline$c(50)-c(51)$ & $1.527(9)$ \\
\hline$c(51)-c(52)$ & $1.493(13)$ \\
\hline$c(52)-c(53)$ & $1.491(12)$ \\
\hline$c(53)-c(54)$ & $1.526(9)$ \\
\hline$c(54)-c(55)$ & $1.407(10)$ \\
\hline$c(55)-c(56)$ & $1.446(10)$ \\
\hline$c(56)-0(4)$ & $1.238(7)$ \\
\hline$c(56)-c(57)$ & $1.498(10)$ \\
\hline$c(57)-c(62)$ & $1.399(10)$ \\
\hline$c(57)-c(58)$ & $1.410(10)$ \\
\hline$c(58)-c(59)$ & $1.420(13)$ \\
\hline$c(59)-c(60)$ & $1.41(2)$ \\
\hline$c(60)-c(61)$ & $1.32(2)$ \\
\hline$c(61)-c(62)$ & $1.434(12)$ \\
\hline$c(63)-c l(2)$ & $1.660(13)$ \\
\hline$c(63)-C l(1)$ & $1.714(11)$ \\
\hline$c(63)-c l\left(2^{\prime}\right)$ & $1.80(2)$ \\
\hline$c(64)-c\left(\left(4^{\prime}\right)\right.$ & $1.63(3)$ \\
\hline$c(64)-c((4)$ & $1.70(3)$ \\
\hline$c(64)-c l(3)$ & $1.72(3)$ \\
\hline$C(23)-N i(1)-C(19)$ & $96.7(3)$ \\
\hline$C(23)-N i(1)-N(1)$ & $148.3(2)$ \\
\hline$C(19)-N i(1)-N(1)$ & $101.4(3)$ \\
\hline$C(23)-N i(1)-N(2)$ & $100.2(2)$ \\
\hline$C(19)-N i(1)-N(2)$ & $143.8(2)$ \\
\hline$N(1)-N i(1)-N(2)$ & $80.0(2)$ \\
\hline$C(23)-N i(1)-C(18)$ & $107.2(3)$ \\
\hline$C(19)-N i(1)-C(18)$ & $40.3(3)$ \\
\hline$N(1)-N i(1)-C(18)$ & $103.4(2)$ \\
\hline$N(2)-N i(1)-C(18)$ & $103.8(2)$ \\
\hline$C(23)-N i(1)-C(24)$ & $41.1(2)$ \\
\hline$C(19)-N i(1)-C(24)$ & $103.4(3)$ \\
\hline$N(1)-N i(1)-C(24)$ & $108.7(2)$ \\
\hline$N(2)-N i(1)-C(24)$ & $110.4(2)$ \\
\hline $\mathrm{C}(18)-\mathrm{Ni}(1)-\mathrm{C}(24)$ & $136.2(3)$ \\
\hline$C(50)-N i(2)-N(4)$ & $100.1(2)$ \\
\hline$C(50)-N i(2)-N(3)$ & $147.0(2)$ \\
\hline$N(4)-N i(2)-N(3)$ & $80.2(2)$ \\
\hline$C(50)-N i(2)-C(49)$ & $40.8(3)$ \\
\hline$N(4)-N i(2)-C(49)$ & $110.8(2)$ \\
\hline $\mathrm{N}(3)-\mathrm{Ni}(2)-\mathrm{C}(49)$ & $107.8(2)$ \\
\hline$C(50)-N i(2)-C(54)$ & $97.6(3)$ \\
\hline$N(4)-N i(2)-C(54)$ & $143.2(2)$ \\
\hline
\end{tabular}




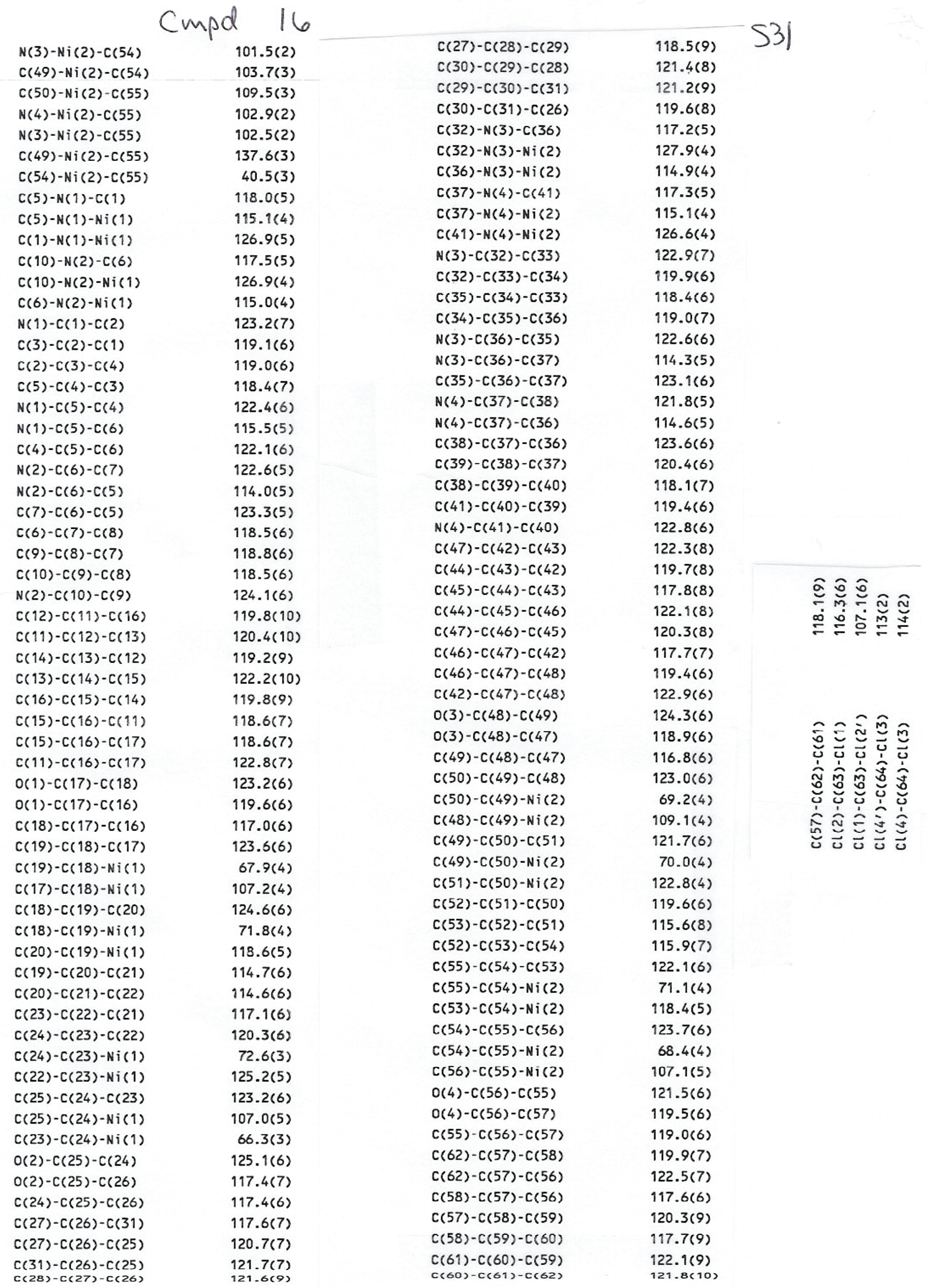




$$
\text { cmpd } 16 \quad 532
$$

Table 4. Anisotropic displacement parameters ( $A^{\wedge} 2 \times 10^{\wedge} 3$ ) for 1 . The anisotropic displacement factor exponent takes the form:

$-2 p^{\wedge} 2\left[h^{\wedge} 2 a^{* \wedge} 2411+\ldots+2 h k a^{*} b^{*}\right.$ u12 ].

\begin{tabular}{|c|c|c|c|c|c|c|}
\hline & U11 & U22 & U33 & U23 & บ13 & U12 \\
\hline $\mathrm{Ni}(1)$ & $40(1)$ & $30(1)$ & $39(1)$ & $0(1)$ & $7(1)$ & $-2(1)$ \\
\hline $\mathrm{Ni}(2)$ & $39(1)$ & $30(1)$ & $37(1)$ & $-2(1)$ & $5(1)$ & $-1(1)$ \\
\hline$N(1)$ & $43(3)$ & $27(2)$ & $47(3)$ & $2(2)$ & $-6(2)$ & $-2(2)$ \\
\hline$N(2)$ & $38(3)$ & $35(2)$ & $34(3)$ & $-9(2)$ & $0(2)$ & $0(2)$ \\
\hline$c(1)$ & $60(4)$ & $29(3)$ & $48(4)$ & $-2(3)$ & $4(3)$ & $-3(3)$ \\
\hline$c(2)$ & $67(5)$ & $30(3)$ & $71(5)$ & $3(3)$ & $-1(4)$ & $2(3)$ \\
\hline$c(3)$ & $44(4)$ & $39(4)$ & $82(5)$ & $19(4)$ & $7(4)$ & $-11(3)$ \\
\hline$c(4)$ & $46(4)$ & $46(4)$ & $45(4)$ & $14(3)$ & $0(3)$ & $-4(3)$ \\
\hline$c(5)$ & $32(3)$ & $35(3)$ & $39(3)$ & $0(3)$ & $3(3)$ & $-2(3)$ \\
\hline$C(6)$ & $30(3)$ & $29(3)$ & $40(4)$ & $6(2)$ & $-1(3)$ & $-3(2)$ \\
\hline$c(7)$ & $43(4)$ & $51(4)$ & $42(4)$ & $2(3)$ & $16(3)$ & $-2(3)$ \\
\hline$C(8)$ & $49(4)$ & $61(4)$ & $41(4)$ & $-13(3)$ & $12(3)$ & $5(3)$ \\
\hline$c(9)$ & $55(4)$ & $45(4)$ & $60(5)$ & $-4(3)$ & $9(4)$ & $0(3)$ \\
\hline$c(10)$ & $49(4)$ & $33(3)$ & $44(4)$ & $-5(3)$ & $3(3)$ & $-4(3)$ \\
\hline$c(11)$ & $57(5)$ & $51(4)$ & $59(5)$ & $-8(4)$ & $-15(4)$ & $8(3)$ \\
\hline$c(12)$ & $83(7)$ & $64(6)$ & $89(7)$ & $-10(6)$ & $-23(6)$ & $13(5)$ \\
\hline$c(13)$ & $81(7)$ & $107(8)$ & $93(8)$ & $-43(7)$ & $-16(6)$ & $30(6)$ \\
\hline$c(14)$ & $71(6)$ & $136(9)$ & $58(6)$ & $0(6)$ & $5(5)$ & $22(6)$ \\
\hline$c(15)$ & $53(5)$ & $82(6)$ & $63(5)$ & $5(4)$ & $2(4)$ & $-4(4)$ \\
\hline$c(16)$ & $31(3)$ & $56(4)$ & $46(4)$ & $-2(3)$ & $-4(3)$ & $5(3)$ \\
\hline$c(17)$ & $32(3)$ & $40(3)$ & $50(4)$ & $9(3)$ & $1(3)$ & $-3(3)$ \\
\hline$O(1)$ & $78(3)$ & $30(2)$ & $58(3)$ & $5(2)$ & $-3(3)$ & $0(2)$ \\
\hline$C(18)$ & $34(4)$ & $33(3)$ & $50(4)$ & $-1(3)$ & $4(3)$ & $2(2)$ \\
\hline$C(19)$ & $46(4)$ & $40(4)$ & $55(4)$ & $-6(3)$ & $10(3)$ & $0(3)$ \\
\hline$c(20)$ & $54(5)$ & $64(5)$ & $64(5)$ & $-1(4)$ & $19(4)$ & $10(3)$ \\
\hline$c(21)$ & $50(4)$ & $72(5)$ & $61(5)$ & $17(4)$ & $24(4)$ & $-8(3)$ \\
\hline$c(22)$ & $63(5)$ & $65(5)$ & $51(5)$ & $7(4)$ & $21(4)$ & $-12(4)$ \\
\hline$c(23)$ & $43(3)$ & $33(3)$ & $45(4)$ & $1(3)$ & $7(3)$ & $-2(3)$ \\
\hline$c(24)$ & $40(4)$ & $41(3)$ & $46(4)$ & $-1(3)$ & $6(3)$ & $-3(3)$ \\
\hline$C(25)$ & $57(4)$ & $50(4)$ & $29(3)$ & $7(3)$ & $-2(3)$ & $-9(3)$ \\
\hline $0(2)$ & $47(3)$ & $56(3)$ & $65(3)$ & $-12(2)$ & $7(2)$ & $5(2)$ \\
\hline$c(26)$ & $63(5)$ & $60(4)$ & $29(4)$ & $-3(3)$ & $8(3)$ & $-13(3)$ \\
\hline$c(27)$ & $55(5)$ & $72(6)$ & $52(5)$ & $-2(4)$ & $0(4)$ & $-11(4)$ \\
\hline$c(28)$ & $63(6)$ & $109(7)$ & $46(5)$ & $-6(4)$ & $4(4)$ & $-28(5)$ \\
\hline$c(29)$ & $115(9)$ & $89(7)$ & $58(5)$ & $-37(5)$ & $33(5)$ & $-67(7)$ \\
\hline$c(30)$ & $91(7)$ & $60(5)$ & $60(5)$ & $-15(4)$ & $14(5)$ & $-17(5)$ \\
\hline$c(31)$ & $73(5)$ & $54(4)$ & $46(4)$ & $-7(3)$ & $0(4)$ & $-10(4)$ \\
\hline$N(3)$ & $41(3)$ & $36(2)$ & $37(3)$ & $-2(2)$ & $8(2)$ & $-1(2)$ \\
\hline$N(4)$ & $32(3)$ & $35(2)$ & $38(3)$ & $2(2)$ & $4(2)$ & $2(2)$ \\
\hline$c(32)$ & $47(4)$ & $45(4)$ & $53(4)$ & $4(3)$ & $-2(3)$ & $-3(3)$ \\
\hline$c(33)$ & $53(4)$ & $30(3)$ & $76(5)$ & $-5(3)$ & $-10(4)$ & $-2(3)$ \\
\hline$c(34)$ & $54(4)$ & $37(3)$ & $66(5)$ & $-10(3)$ & $2(4)$ & $2(3)$ \\
\hline$c(35)$ & $51(4)$ & $40(4)$ & $70(5)$ & $-14(3)$ & $12(4)$ & $1(3)$ \\
\hline$c(36)$ & $33(3)$ & $36(3)$ & $43(4)$ & $-8(3)$ & $-5(3)$ & $0(3)$ \\
\hline$c(37)$ & $33(3)$ & $41(4)$ & $33(3)$ & $-1(3)$ & $-1(3)$ & $-4(2)$ \\
\hline$C(38)$ & $48(4)$ & $49(4)$ & $40(4)$ & $-3(3)$ & $3(3)$ & $1(3)$ \\
\hline$c(39)$ & $48(4)$ & $56(4)$ & $55(4)$ & $5(3)$ & $3(3)$ & $-8(3)$ \\
\hline$C(40)$ & $56(4)$ & $33(3)$ & $51(4)$ & $11(3)$ & $6(3)$ & $-5(3)$ \\
\hline$c(41)$ & $46(4)$ & $38(3)$ & $44(4)$ & $1(3)$ & $4(3)$ & $6(3)$ \\
\hline$c(42)$ & $51(5)$ & $61(5)$ & $44(4)$ & $-7(3)$ & $-1(4)$ & $-4(4)$ \\
\hline
\end{tabular}




\begin{tabular}{|c|c|c|c|c|c|c|c|}
\hline$c(43)$ & $81(6)$ & $56(4)$ & 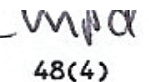 & $\begin{array}{l}16 \\
5(3)\end{array}$ & $2(4)$ & $6(4)$ & \\
\hline$c(44)$ & $94(7)$ & $60(5)$ & $39(4)$ & . $1(3)$ & $1(4)$ & $5(4)$ & \\
\hline$c(45)$ & $54(5)$ & $99(7)$ & $53(5)$ & $-13(4)$ & $-13(4)$ & $35(5)$ & \\
\hline$c(46)$ & $47(4)$ & $72(5)$ & $46(4)$ & $-10(3)$ & $2(3)$ & $1(3)$ & \\
\hline$c(47)$ & $43(4)$ & $48(4)$ & $34(4)$ & $-6(3)$ & $5(3)$ & $8(3)$ & : \\
\hline$c(48)$ & $35(3)$ & $45(3)$ & $42(4)$ & $-6(3)$ & $5(3)$ & $-5(3)$ & \\
\hline $0(3)$ & $41(2)$ & $53(3)$ & $58(3)$ & $-1(2)$ & $8(2)$ & $-6(2)$ & \\
\hline$c(49)$ & $45(4)$ & $48(4)$ & $29(3)$ & $-1(3)$ & $5(3)$ & $-6(3)$ & \\
\hline$c(50)$ & $45(4)$ & $46(4)$ & $43(4)$ & $-15(3)$ & $9(3)$ & $-5(3)$ & \\
\hline$c(51)$ & $53(4)$ & $44(4)$ & $63(5)$ & $-20(3)$ & $7(3)$ & $1(3)$ & \\
\hline$c(52)$ & $106(8)$ & $81(6)$ & $98(7)$ & $3(5)$ & $31(6)$ & $25(5)$ & \\
\hline$c(53)$ & $65(5)$ & $82(6)$ & $48(4)$ & $-3(4)$ & $23(4)$ & $-14(4)$ & \\
\hline$c(54)$ & $39(4)$ & $41(3)$ & $50(4)$ & $-7(3)$ & $13(3)$ & $-19(3)$ & \\
\hline$c(55)$ & $43(4)$ & $31(4)$ & $47(4)$ & $-5(3)$ & $-1(3)$ & $6(3)$ & \\
\hline$c(56)$ & $40(4)$ & $42(4)$ & $45(4)$ & $0(3)$ & $-3(3)$ & $-2(3)$ & \\
\hline $0(4)$ & $63(3)$ & $40(3)$ & $70(3)$ & $-7(2)$ & $-6(3)$ & $4(2)$ & \\
\hline$c(57)$ & $42(4)$ & $42(3)$ & $57(4)$ & $-11(3)$ & $-7(3)$ & $-B(3)$ & \\
\hline$c(58)$ & $50(5)$ & $95(6)$ & $51(5)$ & $-10(4)$ & $7(4)$ & $-9(4)$ & \\
\hline$c(59)$ & $66(6)$ & $128(9)$ & $61(6)$ & $2(6)$ & $10(5)$ & $-1(6)$ & \\
\hline$c(60)$ & $80(6)$ & $152(11)$ & $41(5)$ & $20\langle 6\rangle$ & $-7(5)$ & $-49(7)$ & \\
\hline$c(61)$ & $104(7)$ & $62(5)$ & $78(7)$ & $25(5)$ & $-33(6)$ & $-31(5)$ & \\
\hline$c(62)$ & $80(5)$ & $44(4)$ & $55(4)$ & $4(3)$ & $-5(4)$ & $-11(3)$ & \\
\hline$c(63)$ & $86(7)$ & $94(7)$ & $160(11)$ & $-24(7)$ & $-10(7)$ & $-26(6)$ & \\
\hline$c l(1)$ & $153(3)$ & $93(2)$ & $99(2)$ & $14(2)$ & $29(2)$ & $-15(2)$ & \\
\hline
\end{tabular}


Cmpd le

Table 5. Hydrogen coordinates $\left(x, 10^{\wedge} 4\right)$ and isotropic displacement parameters $\left(A^{\wedge} 2 \times 10^{\wedge} 3\right)$ for 1 .

\begin{tabular}{|c|c|c|c|c|}
\hline & $x$ & $y$ & $z$ & $U(e q)$ \\
\hline$H(10)$ & $-503(5)$ & $3535(4)$ & $10483(3)$ & 51 \\
\hline$H(14)$ & $408(7)$ & $1874(8)$ & $12487(4)$ & 106 \\
\hline$H(35)$ & $1853(5)$ & $2290(4)$ & $15291(3)$ & 64 \\
\hline$H(44)$ & $3265(7)$ & $1286(5)$ & $18229(3)$ & 77 \\
\hline$H(51 A)$ & $-537(5)$ & $4942(4)$ & $17794(3)$ & 64 \\
\hline$H(51 B)$ & $-662(5)$ & $5668(4)$ & $17377(3)$ & 64 \\
\hline$H(52 A)$ & $-2027(7)$ & $4935(6)$ & $17531(4)$ & 113 \\
\hline$H(52 B)$ & $-1820(7)$ & $5012(6)$ & $16928(4)$ & 113 \\
\hline$H(53 A)$ & $-2366(6)$ & $3680(5)$ & $17173(3)$ & 77 \\
\hline$H(53 B)$ & $-1447(6)$ & $3553(5)$ & $17526(3)$ & 77 \\
\hline$H(59)$ & $-333(7)$ & $3630(8)$ & $14032(4)$ & 102 \\
\hline$H(60)$ & $-843(7)$ & $5033(9)$ & $13963(4)$ & 110 \\
\hline$H(1)$ & $-144(47)$ & $-125(41)$ & $9859(27)$ & $49(20)$ \\
\hline$H(2)$ & $-801(86)$ & $-1254(78)$ & $10365(53)$ & $133(44)$ \\
\hline$H(3)$ & $-1696(43)$ & $-1021(38)$ & $10953(24)$ & $41(16)$ \\
\hline$H(4)$ & $-1935(58)$ & $504(52)$ & $11332(34)$ & $88(26)$ \\
\hline$H(7)$ & $-1846(36)$ & $1676(32)$ & $11513(21)$ & $19(13)$ \\
\hline$H(8)$ & $-1927(42)$ & $3079(36)$ & $11803(26)$ & $40(16)$ \\
\hline$H(9)$ & $-1330(53)$ & $4170(50)$ & $11224(32)$ & $78(23)$ \\
\hline$H(11)$ & $1955(47)$ & $2987(40)$ & $11138(28)$ & $43(20)$ \\
\hline$H(12)$ & $1844(50)$ & $3620(48)$ & $11727(30)$ & $54(22)$ \\
\hline$H(13)$ & $-620(52)$ & $3711(45)$ & $12898(29)$ & $78(22)$ \\
\hline$H(15)$ & $648(44)$ & $1035(42)$ & $11748(25)$ & $45(18)$ \\
\hline$H(18)$ & $1611(42)$ & $2383(39)$ & $10379(25)$ & $39(17)$ \\
\hline$H(19)$ & $1234(48)$ & $870(47)$ & $9834(28)$ & $54(21)$ \\
\hline $\mathrm{H}(20 \mathrm{~A})$ & $1353(45)$ & $1445(39)$ & $8940(27)$ & $49(17)$ \\
\hline$H(20 B)$ & $2393(61)$ & $1448(52)$ & $9302(34)$ & $87(25)$ \\
\hline$H(21 A)$ & $2031(45)$ & $2763(42)$ & $8919(28)$ & $56(18)$ \\
\hline$H(21 B)$ & $2078(74)$ & $3550(72)$ & $9184(45)$ & $136(38)$ \\
\hline$H(22 A)$ & $546(39)$ & $2886(35)$ & $8799(25)$ & $35(16)$ \\
\hline$H(22 B)$ & $777(66)$ & $4246(59)$ & $9283(37)$ & $115(32)$ \\
\hline$H(23)$ & $-429(38)$ & $3162(34)$ & $9569(22)$ & $29(14)$ \\
\hline$H(24)$ & $-503(47)$ & $1566(40)$ & $8948(28)$ & 50 \\
\hline$H(27)$ & $-3306(36)$ & $1793(32)$ & $8837(20)$ & $15(13)$ \\
\hline$H(28)$ & $-4154(50)$ & $884(40)$ & $8475(27)$ & $53(18)$ \\
\hline$H(29)$ & $-3254(54)$ & $-471(49)$ & $8427(31)$ & $63(24)$ \\
\hline$H(30)$ & $-1862(45)$ & $-713(46)$ & $8724(26)$ & $48(19)$ \\
\hline$H(31)$ & $-973(43)$ & $328(36)$ & $9159(24)$ & $37(16)$ \\
\hline$H(32)$ & $-5(48)$ & $1955(39)$ & $16718(27)$ & $50(19)$ \\
\hline$H(33)$ & $882(46)$ & $715(45)$ & $16406(27)$ & $58(20)$ \\
\hline$H(34)$ & $1391(58)$ & $1035(50)$ & $15365(35)$ & $82(25)$ \\
\hline$H(38)$ & $1768(68)$ & $3513(68)$ & $14912(42)$ & $117(33)$ \\
\hline$H(39)$ & $1939(41)$ & $4945(35)$ & $14714(26)$ & $41(17)$ \\
\hline$H(40)$ & $1330(35)$ & $5926(35)$ & $15340(21)$ & $25(13)$ \\
\hline$H(41)$ & $571(36)$ & $5545(33)$ & $16099(21)$ & $29(13)$ \\
\hline$H(42)$ & $1157(36)$ & $2418(32)$ & $17595(21)$ & $9(14)$ \\
\hline$H(43)$ & $1654(53)$ & $1210(47)$ & $18068(29)$ & $71(22)$ \\
\hline$H(45)$ & $4056(46)$ & $2477(37)$ & $18013(24)$ & $41(17)$ \\
\hline $\begin{array}{l}H(46) \\
H(49) \\
H(50)\end{array}$ & $\begin{array}{c}3455(37) \\
357(32) \\
418(44)\end{array}$ & $\begin{array}{l}3587(33) \\
3675(28) \\
5230(41)\end{array}$ & $\begin{array}{l}17592(20) \\
17577(19) \\
16867(27)\end{array}$ & $\begin{array}{l}22(13) \\
14(11) \\
54(18)\end{array}$ \\
\hline
\end{tabular}

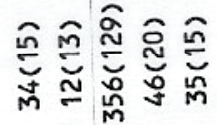

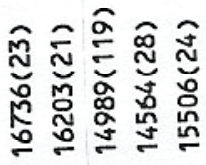

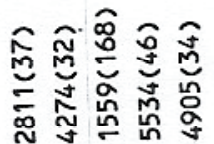
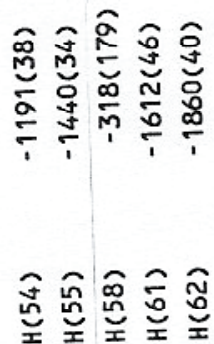


\section{$\$ 35$}

\section{Structure 18}

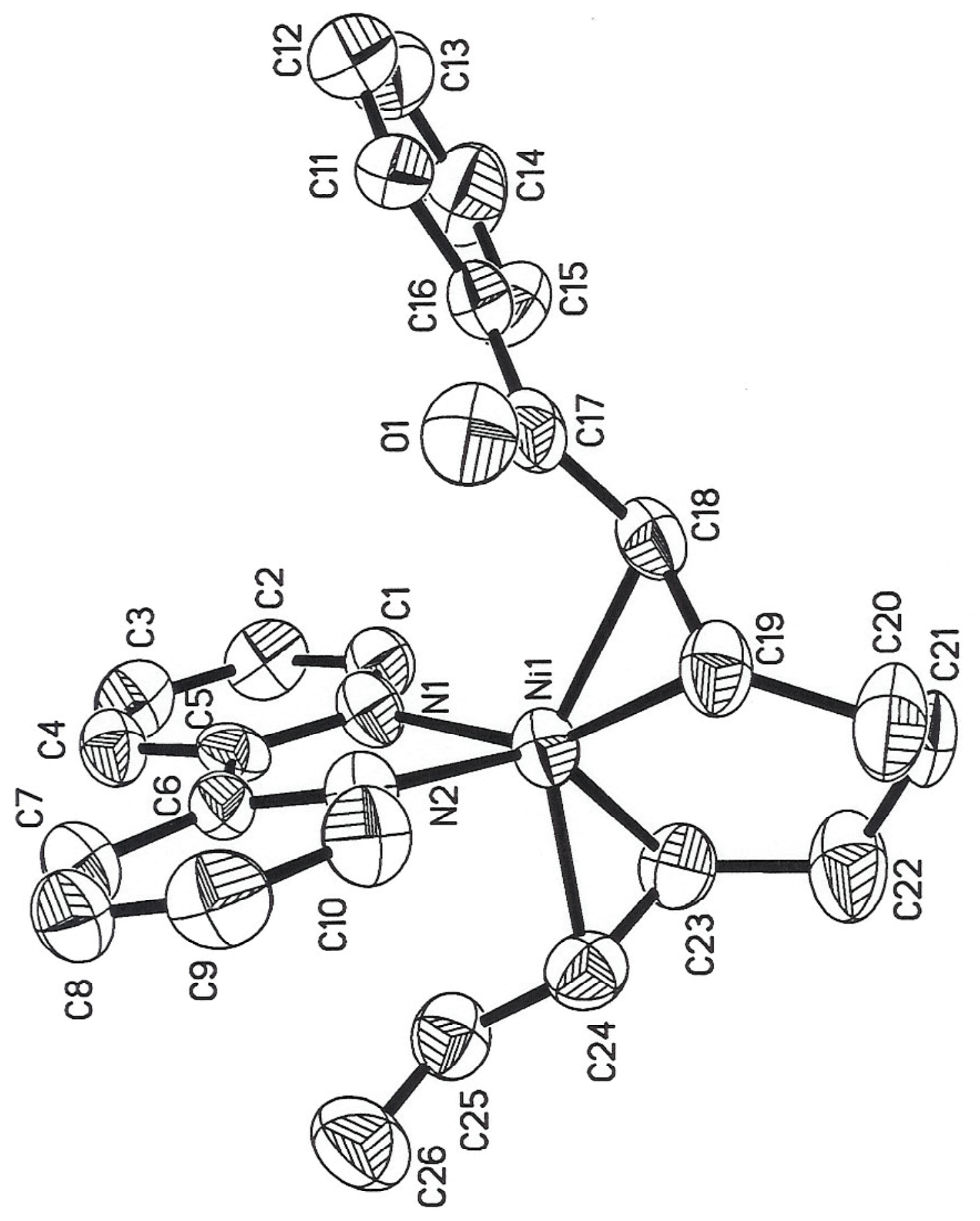




\begin{tabular}{|c|c|}
\hline Ident ification code & rest \\
\hline Empirical formula & C26 H26 N2 Ni O \\
\hline Formula weight & 441.20 \\
\hline Temperature & $295(2) \mathrm{K}$ \\
\hline Wavelength & $0.71073 \mathrm{~A}$ \\
\hline Crystal system & Monoclinic \\
\hline Space group & $P 2(1) / n$ \\
\hline Unit cell dimensions & $\begin{array}{l}a=12.0319(12) \text { A alpha }=90 \text { deg. } \\
b=12.7843(13) \text { A beta }=105.921(3) \mathrm{deg} . \\
c=14.497(2) \text { A gamma }=90 \mathrm{deg} .\end{array}$ \\
\hline Volume & $2144.3(4) A^{\wedge} 3$ \\
\hline$z$ & 4 \\
\hline Density (calculated) & $1.367 \mathrm{Mg} / \mathrm{m}^{\wedge} 3$ \\
\hline Absorption coefficient & $0.925 \mathrm{~mm}^{\wedge}-1$ \\
\hline$F(000)$ & 928 \\
\hline Crystal size & $0.10 \times 0.10 \times 0.10 \mathrm{~mm}$ \\
\hline Theta range for data collection & 2.16 to $28.40 \mathrm{deg}$. \\
\hline Index ranges & $-15<=h<=14,0<=k<=16,0<=l<=18$ \\
\hline Reflections collected & 13986 \\
\hline Independent reflections & $4962[R($ int $)=0.119]$ \\
\hline Absorption correction & Multi-scan \\
\hline Refinement method & Full-matrix least-squares on $\mathrm{F}^{\wedge} 2$ \\
\hline Data / restraints / parameters & $4959 / 0 / 370$ \\
\hline Goodness-of-fit on $\mathrm{F}^{\wedge} 2$ & 0.950 \\
\hline Final $R$ indices $[I>2$ sigma $(I)]$ & $R 1=0.0823, w R 2=0.1241$ \\
\hline$R$ indices (all data) & $R 1=0.2261, w R 2=0.1670$ \\
\hline Extinction coefficient & $0.0011(7)$ \\
\hline Largest diff. peak and hole & 0.296 and -0.322 e. $A^{\wedge}-3$ \\
\hline
\end{tabular}


Table 2. Atomic coordinates $\left(\times 10^{\wedge} 4\right)$ and equivalent isotropic displacement parameters ( $A^{\wedge} 2 \times 10^{\wedge} 3$ ) for $1 . \quad U(e q)$ is defined as one third of the trace of the orthogonalized Uij tensor.

Cimpdol 18

\begin{tabular}{|c|c|c|c|c|}
\hline & $x$ & $y$ & $z$ & $U(e q)$ \\
\hline $\mathrm{Ni}(1)$ & $2338(1)$ & $1718(1)$ & $588(1)$ & $42(1)$ \\
\hline$N(1)$ & $3843(4)$ & $1000(3)$ & $1241(3)$ & $40(1)$ \\
\hline$N(2)$ & $2331(4)$ & $584(3)$ & $-388(3)$ & $39(1)$ \\
\hline$c(1)$ & $4634(5)$ & $1287(5)$ & $2057(4)$ & $45(2)$ \\
\hline$c(2)$ & $5566(6)$ & $674(6)$ & $2483(5)$ & $54(2)$ \\
\hline$c(3)$ & $5718(6)$ & $-271(6)$ & $2076(4)$ & $54(2)$ \\
\hline$c(4)$ & $4927(5)$ & $-565(5)$ & $1247(4)$ & $45(2)$ \\
\hline$c(5)$ & $4001(4)$ & $83(4)$ & $841(4)$ & $38(1)$ \\
\hline$c(6)$ & $3119(5)$ & $-177(4)$ & $-66(4)$ & $37(1)$ \\
\hline$c(7)$ & $3105(6)$ & $-1103(5)$ & $-561(4)$ & $50(2)$ \\
\hline$c(8)$ & $2277(6)$ & $-1257(5)$ & $-1415(5)$ & $57(2)$ \\
\hline$c(9)$ & $1496(6)$ & $-477(6)$ & $-1754(5)$ & $57(2)$ \\
\hline$c(10)$ & $1526(5)$ & $431(5)$ & $-1227(4)$ & $53(2)$ \\
\hline$c(11)$ & $5276(7)$ & $3366(6)$ & $-922(6)$ & $60(2)$ \\
\hline$c(12)$ & $6392(9)$ & $3718(6)$ & $-657(8)$ & $77(3)$ \\
\hline$c(13)$ & $6900(9)$ & $3990(7)$ & $285(9)$ & $84(3)$ \\
\hline$c(14)$ & $6304(7)$ & $3917(6)$ & $943(7)$ & $78(2)$ \\
\hline$c(15)$ & $5179(7)$ & $3582(5)$ & $683(5)$ & $62(2)$ \\
\hline$c(16)$ & $4632(5)$ & $3306(5)$ & $-263(4)$ & $48(2)$ \\
\hline$c(17)$ & $3391(5)$ & $2939(4)$ & $-595(4)$ & $46(2)$ \\
\hline$O(1)$ & $3066(3)$ & $2530(3)$ & $-1403(3)$ & $60(1)$ \\
\hline$C(18)$ & $2641(6)$ & $3121(5)$ & $13(4)$ & $45(2)$ \\
\hline$c(19)$ & $1468(6)$ & $2839(5)$ & $-242(4)$ & $49(2)$ \\
\hline$c(20)$ & $564(7)$ & $3480(7)$ & $53(7)$ & $75(3)$ \\
\hline$c(21)$ & $853(14)$ & $3750(11)$ & $1073(9)$ & $56(5)$ \\
\hline$C\left(21^{\prime}\right)$ & $107(23)$ & $3107(22)$ & $815(15)$ & $89(10)$ \\
\hline$c(22)$ & $863(8)$ & $2831(7)$ & $1716(7)$ & $77(3)$ \\
\hline$c(23)$ & $1689(6)$ & $1938(5)$ & $1717(5)$ & $53(2)$ \\
\hline$c(24)$ & $1315(6)$ & $971(5)$ & $1317(4)$ & $52(2)$ \\
\hline$c(25)$ & $1802(6)$ & $-39(6)$ & $1686(5)$ & $55(2)$ \\
\hline$c(26)$ & $1444(8)$ & $-967(7)$ & $1355(7)$ & $80(2)$ \\
\hline
\end{tabular}


Table 3. Bond lengths [A] and angles [deg] for 1.

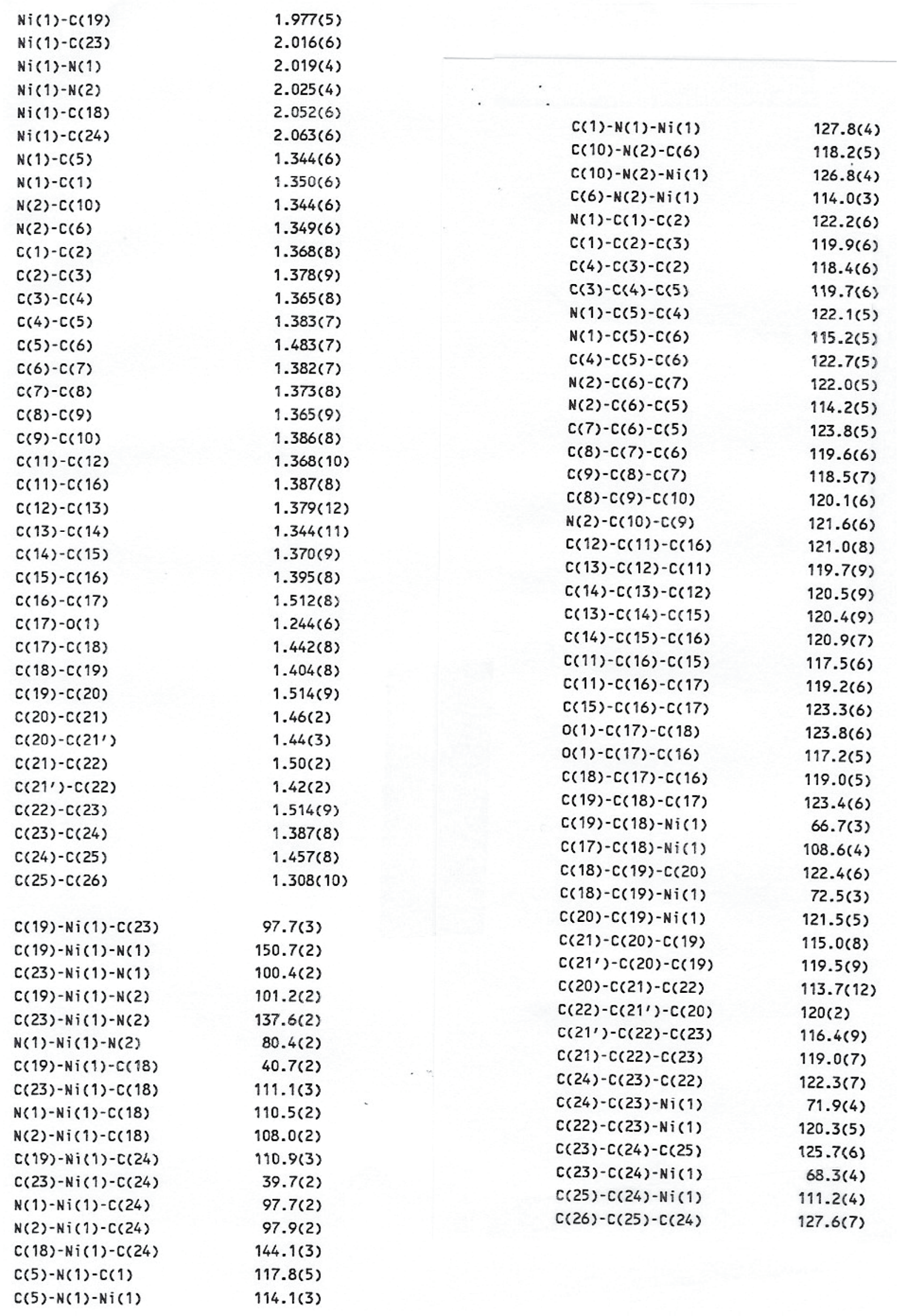


Table 4. Anisotropic displacement parameters ( $A^{\wedge} 2 \times 10^{\wedge} 3$ ) for 1 . The anisotropic displacement factor exponent takes the form: $-2 p^{\wedge} 2$ [ h^2 $a^{\star \wedge} 2$ U11 $+\ldots+2 h k a^{*} b^{\star}$ U12 ]

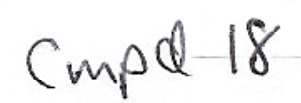

\begin{tabular}{|c|c|c|c|c|c|c|}
\hline & U11 & U22 & U33 & U23 & U13 & U12 \\
\hline $\mathrm{Ni}(1)$ & $48(1)$ & $38(1)$ & $40(1)$ & $1(1)$ & $11(1)$ & $7(1)$ \\
\hline$N(1)$ & $50(3)$ & $34(3)$ & $33(2)$ & $-3(2)$ & $6(2)$ & $2(2)$ \\
\hline$N(2)$ & $42(3)$ & $41(3)$ & $34(3)$ & $-1(2)$ & $8(2)$ & $2(2)$ \\
\hline$c(1)$ & $50(4)$ & $43(4)$ & $42(4)$ & $-3(3)$ & $11(3)$ & $0(3)$ \\
\hline$c(2)$ & $48(5)$ & $72(5)$ & $37(4)$ & $-5(4)$ & $2(3)$ & $-5(4)$ \\
\hline$c(3)$ & $44(4)$ & $67(5)$ & $47(4)$ & $11(4)$ & $5(3)$ & $9(4)$ \\
\hline$c(4)$ & $56(4)$ & $40(4)$ & $39(4)$ & $4(3)$ & $15(3)$ & $8(3)$ \\
\hline$c(5)$ & $39(4)$ & $31(3)$ & $41(3)$ & $11(3)$ & $8(3)$ & $-3(3)$ \\
\hline$c(6)$ & $41(4)$ & $31(3)$ & $40(3)$ & $3(3)$ & $16(3)$ & $2(3)$ \\
\hline$c(7)$ & $58(4)$ & $41(4)$ & $49(4)$ & $-7(3)$ & $12(3)$ & $-2(3)$ \\
\hline$c(8)$ & $68(5)$ & $44(4)$ & $63(5)$ & $-15(4)$ & $26(4)$ & $-7(4)$ \\
\hline$c(9)$ & $58(5)$ & $68(5)$ & $42(4)$ & $-14(4)$ & $6(4)$ & $-13(4)$ \\
\hline$c(10)$ & $54(4)$ & $56(5)$ & $45(4)$ & $1(3)$ & $6(3)$ & $7(3)$ \\
\hline$c(11)$ & $64(5)$ & $47(4)$ & $81(5)$ & $15(4)$ & $40(4)$ & $9(4)$ \\
\hline$C(12)$ & $88(8)$ & $66(6)$ & $95(7)$ & $10(5)$ & $55(6)$ & $7(5)$ \\
\hline$c(13)$ & $62(6)$ & $62(6)$ & $140(10)$ & $-13(5)$ & $48(7)$ & $-10(5)$ \\
\hline$c(14)$ & $56(6)$ & $76(6)$ & $100(7)$ & $-37(5)$ & $17(5)$ & $-7(4)$ \\
\hline$c(15)$ & $63(5)$ & $70(5)$ & $61(5)$ & $-6(4)$ & $32(4)$ & $4(4)$ \\
\hline$c(16)$ & $51(4)$ & $35(3)$ & $60(4)$ & $8(3)$ & $20(3)$ & $12(3)$ \\
\hline$c(17)$ & $58(4)$ & $36(4)$ & $45(4)$ & $12(3)$ & $16(3)$ & $15(3)$ \\
\hline$O(1)$ & $68(3)$ & $71(3)$ & $41(2)$ & $6(2)$ & $12(2)$ & $10(2)$ \\
\hline$c(18)$ & $60(4)$ & $32(4)$ & $44(4)$ & $-3(3)$ & $15(3)$ & $4(3)$ \\
\hline$c(19)$ & $52(4)$ & $51(4)$ & $43(4)$ & $8(3)$ & $9(3)$ & $18(3)$ \\
\hline$c(20)$ & $89(6)$ & $80(6)$ & $69(6)$ & $26(5)$ & $43(5)$ & $47(5)$ \\
\hline$c(21)$ & $53(9)$ & $50(9)$ & $69(9)$ & $-9(7)$ & $25(7)$ & $21(7)$ \\
\hline$c\left(21^{\prime}\right)$ & $63(17)$ & $87(20)$ & $102(17)$ & $-27(13)$ & $-2(12)$ & $49(15)$ \\
\hline$c(22)$ & $107(7)$ & $76(6)$ & $62(6)$ & $11(5)$ & $46(6)$ & $35(5)$ \\
\hline$c(23)$ & $56(5)$ & $58(5)$ & $44(4)$ & $-6(3)$ & $15(3)$ & $4(4)$ \\
\hline$c(24)$ & $50(4)$ & $57(5)$ & $50(4)$ & $9(4)$ & $16(4)$ & $2(4)$ \\
\hline$c(25)$ & $56(5)$ & $58(5)$ & $51(4)$ & $8(4)$ & $12(4)$ & $10(4)$ \\
\hline$c(26)$ & $80(6)$ & $55(6)$ & $98(7)$ & $16(5)$ & $13(5)$ & $2(5)$ \\
\hline
\end{tabular}


Table 5. Hydrogen coordinates $\left(x, 10^{\wedge} 4\right)$ and isotropic displacement parameters $\left(A^{\wedge} 2 \times 10^{\wedge} 3\right)$ for 1 .

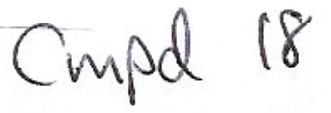

\begin{tabular}{|c|c|c|c|c|}
\hline & $x$ & y & $z$ & $U(e q)$ \\
\hline$H(21 A)$ & $297(14)$ & $4258(11)$ & $1170(9)$ & 67 \\
\hline$H(21 B)$ & $1609(14)$ & $4078(11)$ & $1255(9)$ & 67 \\
\hline$H(21 C)$ & $-410(23)$ & $3643(22)$ & $931(15)$ & 107 \\
\hline$H(21 D)$ & $-365(23)$ & $2499(22)$ & $574(15)$ & 107 \\
\hline$H(1)$ & $4484(40)$ & $2048(40)$ & $2354(33)$ & $49(16)$ \\
\hline$H(2)$ & $6047(39)$ & $886(36)$ & $2967(32)$ & $26(15)$ \\
\hline$H(3)$ & $6383(42)$ & $-625(38)$ & $2374(32)$ & $38(15)$ \\
\hline$H(4)$ & $5050(35)$ & $-1202(36)$ & $949(29)$ & $26(13)$ \\
\hline$H(7)$ & $3711(42)$ & $-1629(39)$ & $-336(33)$ & $44(15)$ \\
\hline$H(8)$ & $2285(45)$ & $-1918(43)$ & $-1784(38)$ & $62(18)$ \\
\hline$H(9)$ & $844(53)$ & $-496(47)$ & $-2263(43)$ & $75(22)$ \\
\hline$H(10)$ & $1007(44)$ & $1073(41)$ & $-1467(33)$ & $51(16)$ \\
\hline$H(11)$ & $4933(56)$ & $3110(52)$ & $-1588(52)$ & $97(27)$ \\
\hline$H(12)$ & $6753(57)$ & $3713(51)$ & $-1060(48)$ & $76(27)$ \\
\hline$H(13)$ & $7563(61)$ & $4147(55)$ & $328(50)$ & $84(28)$ \\
\hline$H(14)$ & $6652(53)$ & $4169(50)$ & $1580(45)$ & $83(23)$ \\
\hline$H(15)$ & $4768(39)$ & $3497(36)$ & $1122(32)$ & $29(14)$ \\
\hline$H(18)$ & $2865(35)$ & $3601(32)$ & $488(28)$ & $17(12)$ \\
\hline$H(19)$ & $1171(39)$ & $2535(37)$ & $-860(34)$ & $41(15)$ \\
\hline$H(20 A)$ & $280(57)$ & $3810(52)$ & $-345(45)$ & $69(26)$ \\
\hline$H(22 A)$ & $741(53)$ & $2760(51)$ & $2183(43)$ & $57(23)$ \\
\hline$H(23)$ & $2333(53)$ & $1963(45)$ & $2204(43)$ & $74(22)$ \\
\hline$H(24)$ & $590(38)$ & $910(32)$ & $865(29)$ & $22(13)$ \\
\hline$H(25)$ & $2429(34)$ & $69(29)$ & $2230(27)$ & $6(11)$ \\
\hline$H(26 A)$ & $1832(51)$ & $-1592(51)$ & $1628(41)$ & $73(21)$ \\
\hline$H(26 B)$ & $766(56)$ & $-1117(50)$ & $813(45)$ & $83(24)$ \\
\hline
\end{tabular}




\section{Cartesian Coordinates and Energies of B3LYP/6-31G(d) Optimized Structures}

\section{Compound 27}

$\mathrm{Ni}$

$\mathrm{O}$

$\mathrm{C}$

$\mathrm{H}$

$\mathrm{C}$

$\mathrm{H}$

$\mathrm{C}$

$\mathrm{H}$

$\mathrm{C}$

$\mathrm{H}$

$\mathrm{H}$

C

$\mathrm{H}$

$\mathrm{H}$

$\mathrm{C}$

$\mathrm{H}$

$\mathrm{H}$

C

C

C

$\mathrm{N}$

C

$\mathrm{H}$

$\mathrm{H}$

C

$\mathrm{H}$

$\mathrm{H}$

$\mathrm{N}$

$\mathrm{H}$

H

H

$\mathrm{H}$

$\mathrm{H}$

$\mathrm{H}$

$\mathrm{H}$

$\mathrm{Ni}$

O

C

C

C

C

C

C

C

$\mathrm{N}$

$\mathrm{N}$
$\mathrm{E}+\mathrm{ZPE}=\mathbf{- 2 1 2 3 . 7 7 8 8 8 9 \text { a.u. }}$

$\begin{array}{llll}-1.02745400 & 0.09913600 & -0.26173100\end{array}$

$\begin{array}{llll}-0.37343100 & 1.64746200 & -0.94354300\end{array}$

$\begin{array}{llll}0.84108400 & 2.18486700 & -0.89733600\end{array}$

$\begin{array}{llll}0.94553100 & 3.04906500 & -1.56471800\end{array}$

$\begin{array}{llll}1.88970600 & 1.82484000 & -0.12856400\end{array}$

$\begin{array}{lll}2.80681700 & 2.40413500 & -0.21747200\end{array}$

$\begin{array}{lll}1.86869800 & 0.62875200 & 0.79531400\end{array}$

$\begin{array}{lll}1.06111600 & 0.74554100 & 1.53759300\end{array}$

$\begin{array}{lll}3.22643500 & 0.39873300 & 1.49084300\end{array}$

$\begin{array}{llll}3.71736300 & 1.33295300 & 1.78828600\end{array}$

$\begin{array}{lll}3.08452100 & -0.20345700 & 2.39928200\end{array}$

$\begin{array}{lll}4.03369800 & -0.40803100 & 0.45823500\end{array}$

$\begin{array}{lll}4.90311800 & -0.91783300 & 0.89007400\end{array}$

$\begin{array}{llll}4.40422800 & 0.26522300 & -0.32463900\end{array}$

$\begin{array}{llll}2.99849600 & -1.38390800 & -0.14099500\end{array}$

$\begin{array}{llll}3.24547300 & -1.66680200 & -1.17138300\end{array}$

$\begin{array}{lll}2.98442100 & -2.31790900 & 0.44205800\end{array}$

$\begin{array}{llll}1.65461400 & -0.65769300 & -0.02055400\end{array}$

$\begin{array}{lll}0.47500600 & -0.97898700 & -0.56662900\end{array}$

$\begin{array}{lll}0.32704100 & -2.18362700 & -1.47622300\end{array}$

$\begin{array}{lll}-2.03200700 & -1.39405500 & 0.43933800\end{array}$

$\begin{array}{lll}-3.20073300 & -0.92039800 & 1.22543800\end{array}$

$\begin{array}{lll}-3.93538500 & -1.71586600 & 1.40346400\end{array}$

$\begin{array}{lll}-2.82570900 & -0.58380400 & 2.19742200\end{array}$

$\begin{array}{lll}-3.81830500 & 0.25710100 & 0.47094800\end{array}$

$\begin{array}{llll}-4.60870600 & 0.73089700 & 1.06733900\end{array}$

$\begin{array}{lll}-4.27279600 & -0.09540300 & -0.46149700\end{array}$

$\begin{array}{lll}-2.71129000 & 1.16619700 & 0.12322700\end{array}$

$\begin{array}{llll}-2.49090600 & 1.80352000 & 0.88898400\end{array}$

$\begin{array}{llll}-2.90831900 & 1.75547100 & -0.68415400\end{array}$

$\begin{array}{lll}-1.41829500 & -1.97647100 & 1.01037800\end{array}$

$\begin{array}{llll}-2.34546100 & -1.99596500 & -0.32608300\end{array}$

$\begin{array}{lll}-0.00695500 & -1.88575500 & -2.48090200\end{array}$

$\begin{array}{llll}-0.41862800 & -2.91399100 & -1.11773700\end{array}$

$\begin{array}{llll}1.26339400 & -2.74395300 & -1.60030500\end{array}$

\section{Compound 28}

$\mathrm{E}+\mathrm{ZPE}=\mathbf{- 2 0 4 6 . 3 9 5 5 2 3}$ a.u.

$\begin{array}{lll}0.60384300 & -0.05605400 & -0.26714300\end{array}$

$\begin{array}{llll}-0.34285800 & -1.40469400 & -1.03002800\end{array}$

$\begin{array}{llll}-1.64280300 & -1.68865100 & -1.05171000\end{array}$

$\begin{array}{llll}-2.61422700 & -1.20840400 & -0.24872100\end{array}$

$\begin{array}{lll}-2.34931600 & -0.14740800 & 0.79586000\end{array}$

$\begin{array}{lll}-3.56059700 & 0.07888600 & 1.70851500\end{array}$

$\begin{array}{llll}-1.90607900 & 1.13171500 & 0.09763500\end{array}$

$\begin{array}{llll}-0.68885000 & 1.29096000 & -0.43504500\end{array}$

$\begin{array}{llll}-0.38774700 & 2.51987200 & -1.26912000\end{array}$

$\begin{array}{lll}2.08744500 & -1.42319900 & -0.08785800\end{array}$

$\begin{array}{lll}1.83785600 & 1.13971200 & 0.61197300\end{array}$ 


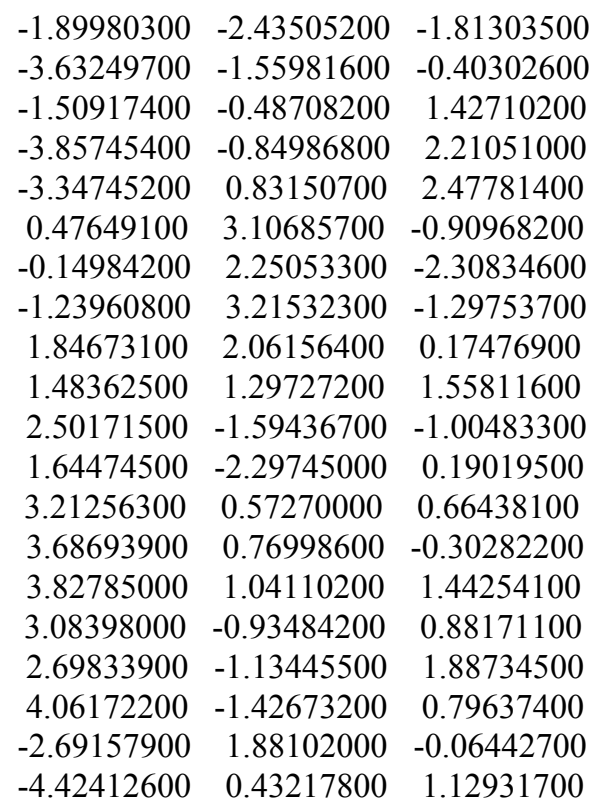

\section{Compound 29}

\begin{tabular}{lrrr} 
& \multicolumn{2}{c}{$\mathbf{E}+\mathbf{Z} \mathbf{E}-\mathbf{- 2 0 4 6 . 3 7 3 3 4 8} \mathbf{a} . \mathbf{u}$} \\
$\mathrm{Ni}$ & -0.33586100 & 0.07208200 & 0.19139500 \\
$\mathrm{O}$ & 2.78895500 & 0.95154800 & -0.61936200 \\
$\mathrm{C}$ & 2.22533300 & 1.39471600 & 0.40353900 \\
$\mathrm{C}$ & 0.84823500 & 1.74727800 & 0.57247600 \\
$\mathrm{C}$ & -0.11167800 & 1.84685200 & -0.49743600 \\
$\mathrm{C}$ & -1.21634000 & 2.87813300 & -0.42513800 \\
$\mathrm{C}$ & -2.00334400 & 0.23194300 & 1.10607200 \\
$\mathrm{C}$ & -2.18727600 & -0.53358500 & 0.10563800 \\
$\mathrm{C}$ & -3.10832000 & -1.35248000 & -0.72165600 \\
$\mathrm{~N}$ & 0.47340500 & -1.58255300 & 1.34093500 \\
$\mathrm{~N}$ & 0.76428100 & -0.81078900 & -1.29245000 \\
$\mathrm{H}$ & 2.84372100 & 1.51904800 & 1.32103800 \\
$\mathrm{H}$ & 0.60253200 & 2.20099100 & 1.53174700 \\
$\mathrm{H}$ & 0.32295200 & 1.77635800 & -1.49925300 \\
$\mathrm{H}$ & -1.60130200 & 2.98969400 & 0.59370700 \\
$\mathrm{H}$ & -0.84217700 & 3.86000000 & -0.75073100 \\
$\mathrm{H}$ & -2.72718400 & -2.37024700 & -0.87989700 \\
$\mathrm{H}$ & -4.10411300 & -1.43745100 & -0.26480400 \\
$\mathrm{H}$ & -3.23714200 & -0.91042900 & -1.71853600 \\
$\mathrm{H}$ & 0.30609300 & -0.79502100 & -2.20096500 \\
$\mathrm{H}$ & 1.61180200 & -0.21758600 & -1.35412300 \\
$\mathrm{H}$ & -0.29654400 & -2.23156400 & 1.50010600 \\
$\mathrm{H}$ & 0.81608900 & -1.30680900 & 2.25848400 \\
$\mathrm{C}$ & 1.13534400 & -2.19018900 & -0.92722000 \\
$\mathrm{H}$ & 0.26143500 & -2.83560500 & -1.07792100 \\
$\mathrm{H}$ & 1.95336500 & -2.58495300 & -1.54602900 \\
$\mathrm{C}$ & 1.54799300 & -2.20675800 & 0.54361900 \\
$\mathrm{H}$ & 2.44695000 & -1.59444700 & 0.66375700 \\
$\mathrm{H}$ & 1.78803700 & -3.23080900 & 0.86254800 \\
$\mathrm{H}$ & -2.06039400 & 2.61503300 & -1.07275900 \\
$\mathrm{H}$ & -2.43512400 & 0.78106100 & 1.93071900
\end{tabular}




\section{Compound 30}

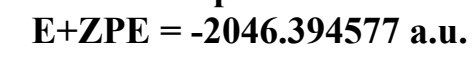

$\mathrm{Ni}$

$\begin{array}{lll}-0.43351100 & 0.16529800 & 0.26892900\end{array}$

$\begin{array}{lll}1.19367400 & -2.76924500 & -0.53897300\end{array}$

$\begin{array}{lll}1.24269400 & -2.07576800 & 0.48227000\end{array}$

$\begin{array}{lll}1.35158500 & -0.62813900 & 0.56566300\end{array}$

$\begin{array}{llll}2.27178700 & 0.09204400 & -0.44130400\end{array}$

$\begin{array}{lll}3.76399500 & -0.03458400 & -0.08838800\end{array}$

$\begin{array}{llll}1.78366900 & 1.51847800 & -0.46142000\end{array}$

$\begin{array}{lll}0.53135500 & 1.74854600 & -0.02908600\end{array}$

$\begin{array}{llll}0.00418600 & 3.16343700 & 0.06754800\end{array}$

$\begin{array}{llll}-1.47934800 & -1.54634200 & 0.13322000\end{array}$

$\begin{array}{llll}-2.16249400 & 1.03293200 & -0.09169900\end{array}$

$\begin{array}{lll}1.08584800 & -2.58790100 & 1.46896300\end{array}$

$\begin{array}{lll}1.62772100 & -0.34706600 & 1.59114700\end{array}$

$2.12892800-0.37481100-1.42698300$

$\begin{array}{lll}4.07883700 & -1.08482400 & -0.08449500\end{array}$

$\begin{array}{llll}4.39199200 & 0.50041900 & -0.81305400\end{array}$

$\begin{array}{llll}-0.84967700 & 3.36241800 & -0.60240300\end{array}$

$\begin{array}{llll}-0.34266400 & 3.40256700 & 1.08468300\end{array}$

$\begin{array}{lll}0.77429800 & 3.90479700 & -0.19062300\end{array}$

$\begin{array}{lll}-2.33340300 & 1.82179200 & 0.53206800\end{array}$

$\begin{array}{llll}-2.09076000 & 1.43803200 & -1.02708100\end{array}$

$\begin{array}{lll}-1.67590100 & -1.98809700 & 1.03447300\end{array}$

$\begin{array}{lll}-0.90843400 & -2.22289300 & -0.38214200\end{array}$

$\begin{array}{llll}-3.28198100 & 0.06001800 & -0.03866900\end{array}$

$\begin{array}{lll}-3.57639000 & -0.05285900 & 1.01033500\end{array}$

$\begin{array}{llll}-4.16204400 & 0.39708100 & -0.60078300\end{array}$

$\begin{array}{lll}-2.74871200 & -1.26326300 & -0.57500800\end{array}$

$\begin{array}{lll}-2.52001300 & -1.16947500 & -1.64183400\end{array}$

$\begin{array}{lll}-3.48883500 & -2.06539300 & -0.46162100\end{array}$

$\begin{array}{lll}2.46122100 & 2.31453400 & -0.79354400\end{array}$

$\begin{array}{lll}3.96962900 & 0.38652600 & 0.90438900\end{array}$

$\mathrm{Ni}$

$\mathrm{O}$

C

C

C

C

C

C

$\mathrm{C}$

$\mathrm{N}$

$\mathrm{N}$

$\mathrm{H}$

$\mathrm{H}$

$\mathrm{H}$

$\mathrm{H}$

$\mathrm{H}$

$\mathrm{H}$

$\mathrm{H}$

$\mathrm{H}$

\section{Compound 31a}

\section{$\mathrm{E}+\mathrm{ZPE}=\mathbf{- 3 9 0 5 . 2 6 2 9 8 4 \text { a.u. }}$}

$\begin{array}{lll}-1.71632300 & 0.12570300 & 0.22287100\end{array}$

$\begin{array}{lll}1.46407800 & 0.77278400 & -0.44481000\end{array}$

$\begin{array}{lll}0.82945500 & 1.38338600 & 0.47454300\end{array}$

$\begin{array}{llll}-0.50167000 & 1.84156900 & 0.42397200\end{array}$

$\begin{array}{llll}-1.36927300 & 1.75257300 & -0.72938300\end{array}$

$\begin{array}{llll}-2.42999900 & 2.80992500 & -0.94273100\end{array}$

$\begin{array}{llll}-3.43295800 & 0.53353300 & 0.98016100\end{array}$

$\begin{array}{lll}-3.61305900 & -0.30695600 & 0.04694500\end{array}$

$\begin{array}{lll}-4.50185500 & -1.13234400 & -0.80513400\end{array}$

$\begin{array}{lll}-1.18686300 & -1.38881900 & 1.63274700\end{array}$

$\begin{array}{lll}-0.59220600 & -1.03189000 & -1.02562900\end{array}$

$\begin{array}{lll}1.37073300 & 1.57465000 & 1.41690700\end{array}$

$\begin{array}{rrr}-0.82397700 & 2.43634300 & 1.27648500\end{array}$

$\begin{array}{llll}-0.84965700 & 1.49794200 & -1.65782000\end{array}$

$\begin{array}{llll}-2.89992600 & 3.11341300 & -0.00161800\end{array}$

$\begin{array}{llll}-1.98449500 & 3.70615300 & -1.39802200\end{array}$

$\begin{array}{lll}-4.19018800 & -2.18466000 & -0.82792600\end{array}$

$\begin{array}{llll}-5.54207400 & -1.09896600 & -0.45444900\end{array}$

$\begin{array}{lll}-4.48813400 & -0.77768700 & -1.84377800\end{array}$ 


$\begin{array}{lrrr}\mathrm{H} & -0.96445600 & -1.09628500 & -1.97108200 \\ \mathrm{H} & 0.31730200 & -0.54731700 & -1.09203600 \\ \mathrm{H} & -2.03572800 & -1.92593000 & 1.80940100 \\ \mathrm{H} & -0.88756800 & -1.02355200 & 2.53483800 \\ \mathrm{C} & -0.40688100 & -2.38343700 & -0.45647200 \\ \mathrm{H} & -1.33064600 & -2.95224100 & -0.61589900 \\ \mathrm{H} & 0.41748700 & -2.92549100 & -0.93555300 \\ \mathrm{C} & -0.13153200 & -2.23916200 & 1.03769500 \\ \mathrm{H} & 0.82576300 & -1.72755400 & 1.17463000 \\ \mathrm{H} & -0.05610600 & -3.22601800 & 1.51312900 \\ \mathrm{H} & -3.22277900 & 2.45845900 & -1.61232700 \\ \mathrm{H} & -3.85061900 & 1.19744100 & 1.72199200 \\ \mathrm{Zn} & 3.43146700 & 0.01995900 & -0.16306800 \\ \mathrm{C} & 4.35799500 & 1.37674500 & 0.89696100 \\ \mathrm{H} & 5.39650400 & 1.09492200 & 1.11335800 \\ \mathrm{H} & 4.39654300 & 2.34872600 & 0.38553600 \\ \mathrm{H} & 3.88321400 & 1.56547300 & 1.87124300 \\ \mathrm{C} & 3.29414400 & -1.72696000 & -1.04909200 \\ \mathrm{H} & 2.87166900 & -2.50351800 & -0.39239800 \\ \mathrm{H} & 2.66367200 & -1.70104600 & -1.95010600 \\ \mathrm{H} & 4.27114800 & -2.11224500 & -1.36899000\end{array}$

\section{Compound 32a}

$\begin{array}{crrr}\mathrm{Ni} & 0.44782400 & 0.25258000 & -0.14584000 \\ \mathrm{O} & 3.48192000 & -0.00242500 & -1.34402800 \\ \mathrm{C} & 2.90268300 & -0.95074700 & -0.83614900 \\ \mathrm{C} & 2.02354200 & -0.88681100 & 0.34305500 \\ \mathrm{C} & 1.97880900 & 0.27415300 & 1.14019900 \\ \mathrm{C} & 1.63026700 & 0.22187300 & 2.60877700 \\ \mathrm{C} & 0.20501100 & 2.05913700 & 0.29993600 \\ \mathrm{C} & -0.84940600 & 1.66596400 & -0.30575400 \\ \mathrm{C} & -2.07226700 & 2.11073100 & -1.03673100 \\ \mathrm{Zn} & -1.59650000 & -0.67619700 & 0.03996000 \\ \mathrm{C} & -0.30704700 & -1.15282200 & -1.53701200 \\ \mathrm{H} & 3.01596200 & -1.96674500 & -1.27743500 \\ \mathrm{H} & 1.70807100 & -1.84549800 & 0.75335500 \\ \mathrm{H} & 2.66807900 & 1.06671800 & 0.85556100 \\ \mathrm{H} & 0.95298500 & -0.60754000 & 2.84038600 \\ \mathrm{H} & 2.54408000 & 0.08042400 & 3.20319200 \\ \mathrm{H} & -2.97138100 & 1.56621300 & -0.72122400 \\ \mathrm{H} & -2.25498700 & 3.17975500 & -0.88073200 \\ \mathrm{H} & -1.95505800 & 1.93762800 & -2.11348000 \\ \mathrm{H} & 1.15719000 & 1.14815900 & 2.95204400 \\ \mathrm{H} & 0.72697800 & 2.84728300 & 0.81913500 \\ \mathrm{C} & -3.26185400 & -1.10369900 & 0.92872600 \\ \mathrm{H} & -3.14677300 & -1.95772100 & 1.60620000 \\ \mathrm{H} & -3.62277200 & -0.25867700 & 1.52681900 \\ \mathrm{H} & -4.05234500 & -1.36212400 & 0.21312300 \\ \mathrm{H} & -0.08311700 & -2.21871100 & -1.42512400 \\ \mathrm{H} & -1.18522100 & -1.07815700 & -2.20089600 \\ \mathrm{H} & 0.50045900 & -0.72461000 & -2.14427800\end{array}$




\section{Compound 32b}

$\begin{array}{lccc}\mathrm{Ni} & 0.64666200 & 0.51070300 & 0.30876800 \\ \mathrm{O} & -0.34600900 & -2.44933500 & -0.05884000 \\ \mathrm{C} & 0.75093800 & -2.37872800 & 0.52400500 \\ \mathrm{C} & 1.60804300 & -1.21713000 & 0.56594800 \\ \mathrm{C} & 1.96208400 & -0.52386400 & -0.65289700 \\ \mathrm{C} & 3.39760400 & -0.09350100 & -0.85497500 \\ \mathrm{C} & 1.15374200 & 2.06108600 & -0.66419700 \\ \mathrm{C} & 0.02602100 & 2.26790000 & -0.12595000 \\ \mathrm{C} & -1.14373200 & 3.10951800 & 0.20452600 \\ \mathrm{C} & -0.80296200 & 0.19902400 & 1.76837200 \\ \mathrm{Zn} & -1.35943500 & -0.58705900 & -0.15384000 \\ \mathrm{H} & 1.10052000 & -3.28205300 & 1.05627400 \\ \mathrm{H} & 2.37620600 & -1.25248000 & 1.33980600 \\ \mathrm{H} & 1.49346500 & -0.89840000 & -1.56597600 \\ \mathrm{H} & 3.82833200 & 0.32375400 & 0.06156500 \\ \mathrm{H} & 4.01502900 & -0.95102500 & -1.15726000 \\ \mathrm{H} & -1.20512300 & 3.28923300 & 1.28445800 \\ \mathrm{H} & -1.08662000 & 4.08083700 & -0.30097400 \\ \mathrm{H} & -2.07812800 & 2.62043300 & -0.09323100 \\ \mathrm{H} & -1.71384000 & 0.80107500 & 1.87658500 \\ \mathrm{H} & -0.05539900 & 0.71868200 & 2.38846600 \\ \mathrm{H} & 3.48735900 & 0.66038200 & -1.64441400 \\ \mathrm{H} & 1.95638300 & 2.37166800 & -1.31150900 \\ \mathrm{H} & -0.96942800 & -0.75296400 & 2.29179600 \\ \mathrm{C} & -2.93736300 & -0.59303100 & -1.29497400 \\ \mathrm{H} & -3.40258500 & 0.39618800 & -1.37755900 \\ \mathrm{H} & -2.68629700 & -0.92258500 & -2.31057200 \\ \mathrm{H} & -3.70154900 & -1.28362500 & -0.91618700\end{array}$

\section{Compound 33}

$\mathrm{Ni}$

$\mathrm{O}$

C

C

C

C

C

$\mathrm{C}$

$\mathrm{C}$

$\mathrm{Zn}$

$\mathrm{C}$

$\mathrm{C}$

$\mathrm{H}$

$\mathrm{H}$

$\mathrm{H}$

$\mathrm{H}$

$\mathrm{H}$

$\mathrm{H}$

H

$\mathrm{H}$

$\mathrm{H}$

$\mathrm{H}$

\section{$\mathbf{E}+\mathrm{ZPE}=\mathbf{- 3 7 1 4 . 8 2 2 5 8 9 \text { a.u. }}$}

$\begin{array}{lll}0.65028100 & 0.33359900 & 0.37734700\end{array}$

$\begin{array}{lll}-0.03037300 & -2.04172300 & -0.47131400\end{array}$

$\begin{array}{lll}0.76894100 & -1.77325700 & 0.55013300\end{array}$

$\begin{array}{llll}2.03064500 & -1.14464900 & 0.45679800\end{array}$

$\begin{array}{lll}2.31123600 & -0.32270100 & -0.65777400\end{array}$

$\begin{array}{lll}3.61848300 & 0.42435800 & -0.74759100\end{array}$

$\begin{array}{llll}0.76790500 & 1.77447300 & -0.88489500\end{array}$

$\begin{array}{llll}-0.30161800 & 1.89387300 & -0.21506100\end{array}$

$\begin{array}{lll}-1.45416400 & 2.72032600 & 0.21392500\end{array}$

$\begin{array}{lll}-1.41284900 & -0.72668800 & -0.33275200\end{array}$

$\begin{array}{lll}-0.44864600 & 0.17590200 & 2.02910800\end{array}$

$\begin{array}{lll}-3.27211300 & -0.35129000 & -0.72647000\end{array}$

$\begin{array}{lll}0.53053500 & -2.25202600 & 1.50648100\end{array}$

$\begin{array}{lll}2.64736200 & -1.11054900 & 1.35299600\end{array}$

$\begin{array}{lll}1.90448500 & -0.65783800 & -1.61042700\end{array}$

$\begin{array}{llll}3.95643500 & 0.77247200 & 0.23473800\end{array}$

$\begin{array}{lll}4.40070600 & -0.22751000 & -1.16021900\end{array}$

$\begin{array}{llll}3.55097500 & 1.29639300 & -1.40709800\end{array}$

$\begin{array}{llll}1.44134200 & 2.12628900 & -1.64747700\end{array}$

$\begin{array}{lll}-1.24300400 & 3.18619800 & 1.18367400\end{array}$

$\begin{array}{llll}-1.64393100 & 3.51915600 & -0.51208600\end{array}$

$\begin{array}{lll}-2.36598500 & 2.12767400 & 0.32691500\end{array}$ 


$\begin{array}{rrrr}H & -0.88735100 & 1.13821500 & 2.30928800 \\ H & 0.24662800 & -0.10600400 & 2.83140700 \\ H & -1.26994100 & -0.55781000 & 2.09636700 \\ H & -3.81038200 & 0.00502700 & 0.15942800 \\ H & -3.37943800 & 0.40809300 & -1.50906100 \\ H & -3.78187400 & -1.25636400 & -1.07661300\end{array}$

$\mathrm{Ni}$

$\mathrm{O}$

C

C

C

C

C

C

C

$\mathrm{N}$

$\mathrm{N}$

$\mathrm{H}$

$\mathrm{H}$

$\mathrm{H}$

$\mathrm{H}$

$\mathrm{H}$

$\mathrm{H}$

$\mathrm{H}$

$\mathrm{H}$

$\mathrm{H}$

$\mathrm{H}$

$\mathrm{H}$

$\mathrm{H}$

C

$\mathrm{H}$

$\mathrm{H}$

C

$\mathrm{H}$

$\mathrm{H}$

$\mathrm{H}$

$\mathrm{H}$

$\mathrm{Zn}$

$\mathrm{C}$

$\mathrm{H}$

$\mathrm{H}$

$\mathrm{H}$

C

$\mathrm{H}$

$\mathrm{H}$

$\mathrm{H}$

$\mathrm{Ni}$

$\mathrm{O}$

C

\section{Compound 34}

\section{$\mathbf{E}+\mathrm{ZPE}=\mathbf{- 3 9 0 5 . 2 8 8 1 8 9}$ a.u.}

$1.36363500-0.17017400-0.07647800$

$\begin{array}{llll}-1.45419600 & 0.99746700 & -0.52077100\end{array}$

$\begin{array}{llll}-0.44414700 & 0.75502700 & -1.22599300\end{array}$

$\begin{array}{llll}0.83463600 & 1.39734300 & -1.16144700\end{array}$

$\begin{array}{llll}0.96370500 & 2.69441400 & -0.36143100\end{array}$

$\begin{array}{llll}2.13890200 & 3.55424300 & -0.85913500\end{array}$

$\begin{array}{lll}1.11504200 & 2.29740900 & 1.08038500\end{array}$

$\begin{array}{lll}1.28251900 & 0.99929300 & 1.38651200\end{array}$

$\begin{array}{lll}1.39354100 & 0.57839500 & 2.83542600\end{array}$

$\begin{array}{lll}1.78455600 & -1.36903700 & -1.63950100\end{array}$

$\begin{array}{lll}1.66241100 & -1.79799400 & 0.99889800\end{array}$

$\begin{array}{lll}-0.55422700 & -0.01867400 & -2.01918100\end{array}$

$\begin{array}{llll}1.36479300 & 1.37697300 & -2.12111200\end{array}$

$\begin{array}{llll}0.04168600 & 3.28752300 & -0.48005900\end{array}$

$\begin{array}{llll}1.99052700 & 3.86997300 & -1.89996100\end{array}$

$\begin{array}{llll}2.25072300 & 4.45778600 & -0.24836200\end{array}$

$\begin{array}{lll}0.61489800 & -0.14619100 & 3.11833500\end{array}$

$\begin{array}{lll}2.36463700 & 0.11306300 & 3.07572900\end{array}$

$\begin{array}{lll}1.28810800 & 1.43607100 & 3.51428700\end{array}$

$\begin{array}{lll}2.15357000 & -1.62583600 & 1.87467700\end{array}$

$\begin{array}{lll}0.72309900 & -2.11959000 & 1.25537200\end{array}$

$2.71824200-1.10984100-1.96533300$

$\begin{array}{lll}1.16813300 & -1.24314600 & -2.44303600\end{array}$

$\begin{array}{lll}2.35277400 & -2.84058900 & 0.20360900\end{array}$

$\begin{array}{lll}3.42098100 & -2.59685800 & 0.18734400\end{array}$

$2.24261300 \quad-3.84325400 \quad 0.63488800$

$\begin{array}{lll}1.77740200 & -2.79044400 & -1.20707500\end{array}$

$\begin{array}{llll}0.73515900 & -3.12387600 & -1.19312700\end{array}$

$2.33610400-3.44373100-1.88839500$

$\begin{array}{lll}1.07492900 & 3.08147000 & 1.84548400\end{array}$

$\begin{array}{llll}3.07996300 & 2.99296100 & -0.79925900\end{array}$

$\begin{array}{lll}-2.95767600 & -0.33428900 & 0.06678200\end{array}$

$\begin{array}{lll}-4.65177200 & 0.61954100 & -0.10245200\end{array}$

$\begin{array}{llll}-4.65043500 & 1.54928100 & 0.48266400\end{array}$

$\begin{array}{llll}-4.85671700 & 0.91198300 & -1.14177600\end{array}$

$\begin{array}{llll}-5.51458600 & 0.03153300 & 0.23670100\end{array}$

$\begin{array}{lll}-1.91111500 & -1.94149100 & 0.54541300\end{array}$

$\begin{array}{llll}-2.52017600 & -2.82800300 & 0.76165800\end{array}$

$\begin{array}{llll}-1.20917500 & -2.22658600 & -0.25460000\end{array}$

$\begin{array}{lll}-1.31847800 & -1.73886800 & 1.45247600\end{array}$

\section{Compound 37a}

$E+Z P E=-3714.816648$ a.u.

$-0.24800500 \quad-0.84786700 \quad-0.48181500$

$\begin{array}{lll}-3.54718400 & -0.73626600 & 0.71077000\end{array}$

$\begin{array}{lll}-2.95373100 & -0.77615800 & -0.35854000\end{array}$ 


$\begin{array}{lrrr}\text { C } & -1.88857400 & 0.13702300 & -0.80305900 \\ \mathrm{C} & -1.80266000 & 1.46555600 & -0.02442300 \\ \mathrm{C} & -1.26898000 & 2.62002800 & -0.89205900 \\ \mathrm{C} & -0.93539100 & 1.17560600 & 1.16573500 \\ \mathrm{C} & -0.11855300 & 0.10094400 & 1.10456500 \\ \mathrm{C} & 0.52662400 & -0.48594200 & 2.34683000 \\ \mathrm{C} & 1.31687400 & -2.03616400 & -0.66183400 \\ \mathrm{Zn} & 1.76108200 & 0.10315400 & -0.15059200 \\ \mathrm{H} & -3.19356500 & -1.59336500 & -1.08591500 \\ \mathrm{H} & -1.93882400 & 0.26216300 & -1.89804600 \\ \mathrm{H} & -2.79958500 & 1.75185000 & 0.34353000 \\ \mathrm{H} & -0.28804600 & 2.36725600 & -1.31471200 \\ \mathrm{H} & -1.95213300 & 2.82733100 & -1.72401000 \\ \mathrm{H} & -0.05567900 & -1.34736500 & 2.69740800 \\ \mathrm{H} & 1.55319800 & -0.85197300 & 2.20422300 \\ \mathrm{H} & 0.54434700 & 0.25497200 & 3.15660100 \\ \mathrm{H} & 0.72450500 & -2.52613200 & -1.46824800 \\ \mathrm{H} & 1.39644600 & -2.75753800 & 0.16035800 \\ \mathrm{H} & -1.15625300 & 3.54115300 & -0.30889500 \\ \mathrm{H} & -1.01950100 & 1.78394600 & 2.07104600 \\ \mathrm{H} & 2.32047200 & -1.97296400 & -1.10813300 \\ \mathrm{C} & 3.34726900 & 1.21175300 & -0.07979300 \\ \mathrm{H} & 3.09864400 & 2.26687500 & -0.23648400 \\ \mathrm{H} & 3.84193900 & 1.12990200 & 0.89529600 \\ \mathrm{H} & 4.07592800 & 0.92379900 & -0.84613100\end{array}$

$\mathrm{Ni}$

C

C

C

C

C

C

C

$\mathrm{Zn}$

$\mathrm{C}$

$\mathrm{H}$

$\mathrm{H}$

$\mathrm{H}$

$\mathrm{H}$

$\mathrm{H}$

$\mathrm{H}$

$\mathrm{H}$

$\mathrm{H}$

$\mathrm{H}$

$\mathrm{H}$

$\mathrm{C}$

$\mathrm{H}$

$\mathrm{H}$

$\mathrm{H}$

H

$\mathrm{H}$

$\mathrm{H}$

\section{Compound 37b}

\section{$\mathbf{E}+\mathrm{ZPE}=\mathbf{- 3 7 1 4 . 8 3 8 3 1 5}$ a.u.}

$\begin{array}{lll}0.31872600 & -0.92928300 & 0.37698100\end{array}$

$\begin{array}{lll}1.13137100 & -1.98182300 & -1.03147500\end{array}$

$2.13074300-1.53822900-0.33570000$

$\begin{array}{lll}2.20876000 & -0.20654600 & 0.10676200\end{array}$

$\begin{array}{lll}1.72594600 & 1.11613400 & -0.50750900\end{array}$

$\begin{array}{lll}2.89824600 & 2.06757000 & -0.79598700\end{array}$

$\begin{array}{lll}0.73049500 & 1.67763800 & 0.50243500\end{array}$

$\begin{array}{lll}-0.02887800 & 0.77200000 & 1.17106600\end{array}$

$\begin{array}{lll}-0.87532300 & 1.15918400 & 2.35860400\end{array}$

$\begin{array}{lll}-1.50583400 & 0.17900200 & -0.42845000\end{array}$

$\begin{array}{lll}-1.47823000 & -1.82303600 & 0.39252000\end{array}$

$\begin{array}{lll}2.80737800 & -2.27438900 & 0.12551800\end{array}$

$\begin{array}{lll}2.92359800 & -0.05730100 & 0.91678400\end{array}$

$\begin{array}{llll}1.22562400 & 0.90829300 & -1.46594600\end{array}$

$\begin{array}{lll}3.44745400 & 2.30604100 & 0.12296100\end{array}$

$\begin{array}{llll}3.60059300 & 1.61617500 & -1.50444900\end{array}$

$\begin{array}{lll}-1.88187300 & 0.71948300 & 2.33868500\end{array}$

$\begin{array}{lll}-0.40866000 & 0.78322400 & 3.27900100\end{array}$

$\begin{array}{lll}-0.98280200 & 2.24837800 & 2.46078700\end{array}$

$\begin{array}{llll}2.53834500 & 3.00920600 & -1.22686300\end{array}$

$\begin{array}{lll}0.70241100 & 2.75480100 & 0.69804100\end{array}$

$\begin{array}{llll}-2.74970000 & 1.21905200 & -1.48913300\end{array}$

$\begin{array}{llll}-2.24030400 & 2.04917700 & -1.99109200\end{array}$

$\begin{array}{llll}-3.22906400 & 0.60693000 & -2.26125700\end{array}$

$\begin{array}{llll}-3.54372200 & 1.64785500 & -0.86614300\end{array}$

$\begin{array}{lll}-1.81195500 & -2.36697900 & -0.49798300\end{array}$

$\begin{array}{llll}-2.36193600 & -1.54221900 & 0.98481500\end{array}$

$\begin{array}{lll}-0.95772300 & -2.56683400 & 1.01861900\end{array}$ 


\section{Compound 38a}

$\mathrm{Ni}$

$\mathrm{O}$

C

C

C

C

C

C

C

$\mathrm{Zn}$

C

$\mathrm{H}$

$\mathrm{H}$

$\mathrm{H}$

$\mathrm{H}$

$\mathrm{H}$

$\mathrm{H}$

$\mathrm{H}$

H

$\mathrm{H}$

$\mathrm{H}$

C

$\mathrm{H}$

$\mathrm{H}$

$\mathrm{H}$

$\mathrm{H}$

$\mathrm{H}$

H

$\mathrm{Ni}$

$\mathrm{O}$

$\mathrm{C}$

C

C

C

$\mathrm{C}$

C

C

$\mathrm{C}$

$\mathrm{Zn}$

$\mathrm{H}$

H

$\mathrm{H}$

$\mathrm{H}$

H

$\mathrm{H}$

H

$\mathrm{H}$

H

$\mathrm{H}$

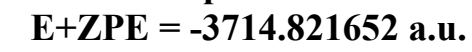

$\begin{array}{llll}0.04780200 & -0.59460700 & 0.45207300\end{array}$

$\begin{array}{llll}-0.98288700 & -1.74924800 & -0.40714000\end{array}$

$\begin{array}{llll}-2.15980400 & -1.68724800 & -1.02578900\end{array}$

$\begin{array}{lll}-3.09012300 & -0.74101000 & -0.78664400\end{array}$

$\begin{array}{lll}-2.84184300 & 0.35509700 & 0.21761000\end{array}$

$\begin{array}{lll}-4.11671400 & 1.14139500 & 0.54771400\end{array}$

$\begin{array}{llll}-1.73863300 & 1.28395100 & -0.29173600\end{array}$

$\begin{array}{llll}-0.42784900 & 1.03015000 & -0.29847800\end{array}$

$\begin{array}{llll}0.56183100 & 2.04315200 & -0.82673900\end{array}$

$\begin{array}{llll}2.14676500 & -0.03202700 & -0.03012700\end{array}$

$\begin{array}{lll}1.19317900 & -0.04463100 & 1.95965600\end{array}$

$\begin{array}{lll}-2.32312400 & -2.47372900 & -1.76721600\end{array}$

$\begin{array}{lll}-4.00763200 & -0.73682600 & -1.36983100\end{array}$

$\begin{array}{lll}-2.48289500 & -0.10919400 & 1.15100200\end{array}$

$\begin{array}{lll}-4.89468900 & 0.47630800 & 0.93821700\end{array}$

$\begin{array}{llll}-3.92229800 & 1.91837200 & 1.29601600\end{array}$

$\begin{array}{llll}1.27930600 & 2.36997100 & -0.05713500\end{array}$

$\begin{array}{llll}1.14165500 & 1.66921700 & -1.68278900\end{array}$

$\begin{array}{llll}0.04837800 & 2.95101800 & -1.17013600\end{array}$

$\begin{array}{lll}-2.09212600 & 2.22331700 & -0.72883900\end{array}$

$\begin{array}{llll}-4.51583300 & 1.63228400 & -0.34921200\end{array}$

$\begin{array}{llll}3.85147100 & 0.13072300 & -0.92214700\end{array}$

$\begin{array}{llll}4.49901400 & -0.72664000 & -0.70907000\end{array}$

$\begin{array}{llll}3.71890500 & 0.18302600 & -2.00836700\end{array}$

$\begin{array}{llll}4.38012200 & 1.03771000 & -0.60561700\end{array}$

$\begin{array}{llll}1.46292300 & -0.96646000 & 2.49759200\end{array}$

$\begin{array}{lll}2.05041000 & 0.63725700 & 2.10315300\end{array}$

$\begin{array}{lll}0.39046400 & 0.44866400 & 2.52446400\end{array}$

\section{Compound 38b}

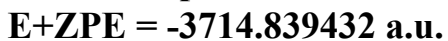

$\begin{array}{lll}-0.13508200 & -1.29972100 & -0.32990800\end{array}$

$\begin{array}{lll}1.56391000 & -1.69625500 & -0.65974900\end{array}$

$\begin{array}{lll}2.72437700 & -1.06345000 & -0.53867700\end{array}$

$\begin{array}{llll}2.88895800 & 0.25661000 & -0.30102600\end{array}$

$\begin{array}{lll}1.72151700 & 1.19697000 & -0.12728100\end{array}$

$\begin{array}{lll}2.15081000 & 2.66842000 & -0.10179900\end{array}$

$\begin{array}{lll}0.95839000 & 0.79713500 & 1.12662400\end{array}$

$\begin{array}{lll}0.06641900 & -0.21434000 & 1.17479600\end{array}$

$\begin{array}{lll}-0.51354700 & -0.69989300 & 2.48586000\end{array}$

$\begin{array}{lll}-2.05093500 & -1.57249400 & -0.44540300\end{array}$

$\begin{array}{llll}-1.37468000 & 0.61747900 & -0.21212100\end{array}$

$\begin{array}{lll}3.59622700 & -1.71258400 & -0.65734400\end{array}$

$\begin{array}{llll}3.89800400 & 0.64828300 & -0.20387800\end{array}$

$\begin{array}{llll}1.03247800 & 1.05017000 & -0.98250200\end{array}$

$\begin{array}{llll}2.68095400 & 2.93147700 & -1.02321300\end{array}$

$\begin{array}{lll}1.28838800 & 3.33713200 & 0.00071900\end{array}$

$\begin{array}{lll}-1.59642900 & -0.86230200 & 2.44211500\end{array}$

$\begin{array}{lll}-0.06902700 & -1.66338500 & 2.76624100\end{array}$

$\begin{array}{lll}-0.30840500 & 0.00870600 & 3.29902400\end{array}$

$\begin{array}{llll}-2.69369800 & -1.58230200 & 0.44450600\end{array}$

$\begin{array}{llll}-1.87336500 & -2.64130200 & -0.68428200\end{array}$ 


$\begin{array}{lrrr}\mathrm{H} & 1.23825600 & 1.32033400 & 2.04556000 \\ \mathrm{H} & 2.82804100 & 2.86132100 & 0.73958300 \\ \mathrm{H} & -2.66105400 & -1.19993000 & -1.28129800 \\ \mathrm{C} & -2.43099900 & 2.19443800 & -0.60365800 \\ \mathrm{H} & -1.79251500 & 3.07965100 & -0.70143400 \\ \mathrm{H} & -2.99329200 & 2.08566700 & -1.53786100 \\ \mathrm{H} & -3.15307900 & 2.39655900 & 0.19649900\end{array}$

$\mathrm{Ni}$

$\mathrm{O}$

C

$\mathrm{C}$

C

C

C

C

C

$\mathrm{Zn}$

C

$\mathrm{C}$

$\mathrm{H}$

H

H

$\mathrm{H}$

$\mathrm{H}$

H

H

H

H

$\mathrm{H}$

$\mathrm{H}$

$\mathrm{H}$

$\mathrm{H}$

H

H

$\mathrm{H}$

$\mathrm{Ni}$

$\mathrm{O}$

C

C

C

$\mathrm{C}$

$\mathrm{C}$

C

C

N

$\mathrm{N}$

$\mathrm{H}$

$\mathrm{H}$

$\mathrm{H}$

\section{Compound 38c}

\section{$\mathrm{E}+\mathrm{ZPE}=\mathbf{- 3 7 1 4 . 8 4 5 9 4 9 \text { a.u. }}$}

$\begin{array}{lll}1.46458500 & -0.63909900 & -0.40292400\end{array}$

$\begin{array}{llll}-0.73622000 & 0.49687300 & -1.81748900\end{array}$

$\begin{array}{llll}0.54635600 & 0.78002000 & -1.75213000\end{array}$

$\begin{array}{llll}1.21693600 & 1.40307900 & -0.70709300\end{array}$

$\begin{array}{lll}0.47474300 & 1.92129000 & 0.51366100\end{array}$

$\begin{array}{lll}1.39106400 & 2.75030300 & 1.43141800\end{array}$

$\begin{array}{lll}-0.10482300 & 0.74237900 & 1.27533200\end{array}$

$\begin{array}{lll}0.29740000 & -0.53212300 & 1.00330500\end{array}$

$\begin{array}{lll}-0.13380700 & -1.66843100 & 1.89077700\end{array}$

$\begin{array}{lll}-1.70034800 & -0.00184800 & -0.28387900\end{array}$

$\begin{array}{llll}2.13654300 & -2.35127500 & -0.04603200\end{array}$

$\begin{array}{rrr}-3.48182500 & -0.53507300 & 0.26340900\end{array}$

$\begin{array}{llll}1.09870800 & 0.62297100 & -2.69152700\end{array}$

$\begin{array}{llll}2.19538200 & 1.82505600 & -0.93386900\end{array}$

$\begin{array}{lll}-0.34394400 & 2.58504100 & 0.18352300\end{array}$

$\begin{array}{lll}2.23920100 & 2.14626200 & 1.77363100\end{array}$

$\begin{array}{lll}1.78111000 & 3.62654600 & 0.90085200\end{array}$

$\begin{array}{llll}0.84870400 & 3.10430700 & 2.31506200\end{array}$

$\begin{array}{lll}-0.72948000 & 0.95714800 & 2.14971700\end{array}$

$\begin{array}{lll}0.73440900 & -2.06465400 & 2.43052600\end{array}$

$\begin{array}{lll}-0.88033200 & -1.35113000 & 2.63131000\end{array}$

$\begin{array}{lll}-0.54767700 & -2.50814500 & 1.32139000\end{array}$

$\begin{array}{lll}2.72774000 & -2.43006700 & 0.87567100\end{array}$

$\begin{array}{llll}2.84000700 & -2.47681300 & -0.89879600\end{array}$

$\begin{array}{lll}1.43524800 & -3.19321200 & -0.07494600\end{array}$

$\begin{array}{lll}-3.89664400 & -1.29498700 & -0.40890700\end{array}$

$\begin{array}{lll}-3.49414600 & -0.95115000 & 1.27711100\end{array}$

$\begin{array}{llll}-4.17199200 & 0.31705800 & 0.25154400\end{array}$

\section{Compound TS-28}

$\mathrm{E}+\mathrm{ZPE}=\mathbf{- 2 0 4 6 . 3 6 2 9 8 7 \text { a.u. }}$

$-0.39147400 \quad-0.05262700 \quad-0.06986900$

$\begin{array}{llll}0.30628500 & -1.17104200 & -1.80169500\end{array}$

$\begin{array}{lll}0.67572600 & -1.77994900 & -0.71925200\end{array}$

$\begin{array}{lll}1.54025300 & -1.37729500 & 0.29863800\end{array}$

$\begin{array}{lll}2.66230800 & -0.35997700 & 0.21271800\end{array}$

$\begin{array}{lll}3.40384200 & -0.24034200 & 1.55884300\end{array}$

$\begin{array}{lll}2.18524800 & 0.98154400 & -0.26333900\end{array}$

$\begin{array}{llll}0.88745100 & 1.27764600 & -0.39425200\end{array}$

$\begin{array}{llll}0.47651600 & 2.62400800 & -0.95078600\end{array}$

$\begin{array}{llll}-1.95895600 & -1.34670900 & 0.01688000\end{array}$

$\begin{array}{lll}-1.65364400 & 1.25741100 & 0.59616900\end{array}$

$\begin{array}{lll}0.19471200 & -2.76540300 & -0.51114800\end{array}$

$\begin{array}{lll}1.63729800 & -2.11312900 & 1.09709800\end{array}$

$3.40266800-0.72823200-0.52238200$ 


$\begin{array}{rrrr}\mathrm{H} & 2.71817100 & 0.10763200 & 2.34104500 \\ \mathrm{H} & 3.81921000 & -1.20705500 & 1.87212800 \\ \mathrm{H} & -0.15991200 & 2.51677000 & -1.84217500 \\ \mathrm{H} & -0.08017900 & 3.25189300 & -0.23488300 \\ \mathrm{H} & 1.35320800 & 3.21268800 & -1.25369400 \\ \mathrm{H} & -1.21224400 & 1.81496200 & 1.32729900 \\ \mathrm{H} & -1.90245800 & 1.92139800 & -0.13980700 \\ \mathrm{H} & -1.89356300 & -2.06105500 & 0.74127700 \\ \mathrm{H} & -1.91501100 & -1.82384100 & -0.88419500 \\ \mathrm{C} & -2.87799000 & 0.59300200 & 1.11242200 \\ \mathrm{H} & -2.64762900 & 0.20218000 & 2.10886800 \\ \mathrm{H} & -3.71932600 & 1.29123900 & 1.20493400 \\ \mathrm{C} & -3.19876100 & -0.55653900 & 0.16443700 \\ \mathrm{H} & -3.46580300 & -0.16217600 & -0.82188000 \\ \mathrm{H} & -4.04775300 & -1.14755500 & 0.53025400 \\ \mathrm{H} & 4.23138000 & 0.47614100 & 1.49069200 \\ \mathrm{H} & 2.95886300 & 1.70791000 & -0.54145500\end{array}$

$\mathrm{Ni}$

$\mathrm{O}$

C

C

$\mathrm{C}$

C

$\mathrm{C}$

C

C

N

$\mathrm{N}$

$\mathrm{H}$

$\mathrm{H}$

H

H

H

H

$\mathrm{H}$

$\mathrm{H}$

$\mathrm{H}$

$\mathrm{H}$

H

$\mathrm{H}$

$\mathrm{C}$

$\mathrm{H}$

$\mathrm{H}$

C

H

H

H

$\mathrm{H}$

$\mathrm{Ni}$

\section{Compound TS-30}

$\mathrm{E}+\mathrm{ZPE}=\mathbf{- 2 0 4 6 . 3 2 4 2 5 6}$ a.u.

$-0.13786200 \quad-0.46169200 \quad-0.14611900$

$\begin{array}{lll}1.80912800 & 2.30499600 & 0.19699400\end{array}$

$\begin{array}{lll}0.92238700 & 2.08858900 & 1.08603800\end{array}$

$\begin{array}{lll}-0.36407000 & 1.56178700 & 0.94581000\end{array}$

$\begin{array}{llll}-1.09984900 & 1.43992400 & -0.33125600\end{array}$

$\begin{array}{llll}-2.44054500 & 2.18262700 & -0.27451900\end{array}$

$\begin{array}{lll}-1.95144400 & -0.23887500 & -0.84291100\end{array}$

$\begin{array}{lll}-1.82176600 & -1.20652800 & 0.02547700\end{array}$

$\begin{array}{lll}-2.58613500 & -2.37694100 & 0.53048500\end{array}$

$\begin{array}{lll}1.47761600 & 0.08563500 & -1.22133500\end{array}$

$\begin{array}{lll}1.07654200 & -1.67289300 & 0.79507700\end{array}$

$\begin{array}{lll}1.19263700 & 2.33790900 & 2.13490500\end{array}$

$\begin{array}{lll}-0.97069300 & 1.53640700 & 1.84921500\end{array}$

$\begin{array}{lll}-0.52551400 & 1.80820300 & -1.18483200\end{array}$

$\begin{array}{llll}-3.07580500 & 1.80352800 & 0.53213100\end{array}$

$\begin{array}{llll}-2.22182900 & 3.23811900 & -0.06614100\end{array}$

$-2.82329000 \quad-2.25754200 \quad 1.59701400$

$\begin{array}{lll}-3.53571000 & -2.52084200 & -0.00314800\end{array}$

$\begin{array}{lll}-2.00951600 & -3.30774300 & 0.44410000\end{array}$

$\begin{array}{lll}0.87419000 & -1.66484100 & 1.79397500\end{array}$

$\begin{array}{lll}0.96807100 & -2.64147300 & 0.49214500\end{array}$

$\begin{array}{llll}1.70966900 & 1.04962900 & -0.84810100\end{array}$

$\begin{array}{lll}1.32734600 & 0.15819000 & -2.22560400\end{array}$

$\begin{array}{lll}2.47424700 & -1.21704500 & 0.54705400\end{array}$

$\begin{array}{lll}2.63817400 & -0.32385400 & 1.15562400\end{array}$

$\begin{array}{lll}3.21124500 & -1.97918700 & 0.83015300\end{array}$

$\begin{array}{lll}2.58433200 & -0.84099000 & -0.92664500\end{array}$

$\begin{array}{lll}2.48536900 & -1.73796700 & -1.55164500\end{array}$

$3.57031300 \quad-0.39950100 \quad-1.12586100$

$\begin{array}{llll}-2.60309100 & 0.01389300 & -1.67074400\end{array}$

$\begin{array}{llll}-3.00650100 & 2.13000100 & -1.21119700\end{array}$

Compound TS-33

$\mathbf{E}+\mathrm{ZPE}=\mathbf{- 3 7 1 4 . 8 2 2 5 8 1}$ a.u.

$\begin{array}{lll}0.66035400 & 0.37756400 & 0.32674500\end{array}$ 


$\begin{array}{lrrr}\mathrm{O} & -0.17125200 & -2.15477900 & -0.56303100 \\ \mathrm{C} & 0.66496800 & -2.08557900 & 0.39608800 \\ \mathrm{C} & 1.83512900 & -1.28627000 & 0.45704100 \\ \mathrm{C} & 2.22932900 & -0.42016600 & -0.60295600 \\ \mathrm{C} & 3.62010300 & 0.17045400 & -0.58571000 \\ \mathrm{C} & 1.01175000 & 1.86361700 & -0.81891600 \\ \mathrm{C} & -0.09264500 & 2.04306100 & -0.22522900 \\ \mathrm{C} & -1.24121100 & 2.89921500 & 0.14742200 \\ \mathrm{C} & -0.67981900 & 0.13017400 & 1.85693400 \\ \mathrm{Zn} & -1.41870800 & -0.55861700 & -0.17138800 \\ \mathrm{C} & -3.22176200 & -0.51755400 & -0.90256300 \\ \mathrm{H} & 0.47761600 & -2.71475600 & 1.28418900 \\ \mathrm{H} & 2.43623300 & -1.36604000 & 1.36068100 \\ \mathrm{H} & 1.86901300 & -0.69604500 & -1.59419600 \\ \mathrm{H} & 3.92289900 & 0.46811800 & 0.42431200 \\ \mathrm{H} & 4.34797300 & -0.56766600 & -0.94970000 \\ \mathrm{H} & 3.70469600 & 1.04943400 & -1.23307800 \\ \mathrm{H} & 1.75837400 & 2.17722700 & -1.52797100 \\ \mathrm{H} & -1.15229000 & 3.22993200 & 1.18899300 \\ \mathrm{H} & -1.28314100 & 3.78872000 & -0.49151600 \\ \mathrm{H} & -2.19185800 & 2.36391700 & 0.05710700 \\ \mathrm{H} & -1.53496700 & 0.80622600 & 1.96999600 \\ \mathrm{H} & 0.07868300 & 0.51008700 & 2.55986500 \\ \mathrm{H} & -0.97655200 & -0.83747100 & 2.28784900 \\ \mathrm{H} & -3.72976800 & 0.44019300 & -0.74414300 \\ \mathrm{H} & -3.19947300 & -0.70925500 & -1.98145900 \\ \mathrm{H} & -3.84114200 & -1.29937400 & -0.44655700\end{array}$

$\begin{array}{lrrr}\mathrm{Ni} & -1.78964100 & -0.35570500 & -0.08468400 \\ \mathrm{O} & 1.41711900 & 0.78521200 & -0.30585500 \\ \mathrm{C} & 0.66011400 & 1.28850000 & 0.62379500 \\ \mathrm{C} & -0.68363700 & 1.58270900 & 0.53828100 \\ \mathrm{C} & -1.48176900 & 1.63884700 & -0.71166500 \\ \mathrm{C} & -2.13035700 & 3.01807700 & -0.88195800 \\ \mathrm{C} & -3.23184300 & 0.72858100 & -0.81154300 \\ \mathrm{C} & -3.54785100 & 0.09268400 & 0.28057300 \\ \mathrm{C} & -4.73030700 & -0.25588600 & 1.10910800 \\ \mathrm{~N} & -0.30740100 & -1.09561500 & -1.22887400 \\ \mathrm{~N} & -1.44337100 & -1.88796100 & 1.09262600 \\ \mathrm{H} & 1.14346900 & 1.49833900 & 1.58970100 \\ \mathrm{H} & -1.12534100 & 2.06092800 & 1.40974600 \\ \mathrm{H} & -0.88974300 & 1.41726300 & -1.60236900 \\ \mathrm{H} & -2.78590300 & 3.26545300 & -0.04136700 \\ \mathrm{H} & -1.32052400 & 3.75860300 & -0.90969200 \\ \mathrm{H} & -4.66261200 & 0.21108700 & 2.10127100 \\ \mathrm{H} & -5.67161000 & 0.07608200 & 0.65142300 \\ \mathrm{H} & -4.80444600 & -1.33820200 & 1.27895000 \\ \mathrm{H} & -1.46938000 & -1.60535200 & 2.07188900 \\ \mathrm{H} & -2.15887100 & -2.60870900 & 0.98997000 \\ \mathrm{H} & 0.50519400 & -0.45719000 & -1.07115800 \\ \mathrm{H} & -0.49723100 & -1.10104300 & -2.22971400 \\ \mathrm{C} & -0.10501800 & -2.45553400 & 0.75431100 \\ \mathrm{H} & 0.64945200 & -1.79051300 & 1.18343300 \\ \mathrm{H} & 0.03360200 & -3.45781900 & 1.17712100\end{array}$




$\begin{array}{lrrr}\mathrm{C} & 0.02624000 & -2.45813100 & -0.76312600 \\ \mathrm{H} & -0.68597200 & -3.16871900 & -1.20066700 \\ \mathrm{H} & 1.03840800 & -2.75891600 & -1.05669100 \\ \mathrm{H} & -3.71179300 & 1.16540500 & -1.67756000 \\ \mathrm{H} & -2.70654700 & 3.11225000 & -1.80855700 \\ \mathrm{Zn} & 3.38677500 & 0.22934600 & 0.04001900 \\ \mathrm{C} & 4.09666400 & 1.39899700 & 1.44745100 \\ \mathrm{H} & 5.16514100 & 1.22444400 & 1.63057600 \\ \mathrm{H} & 3.99158800 & 2.46568500 & 1.20373800 \\ \mathrm{H} & 3.59634900 & 1.25990700 & 2.41783100 \\ \mathrm{C} & 3.60040100 & -1.33304300 & -1.14422500 \\ \mathrm{H} & 3.36166000 & -2.28044900 & -0.63443200 \\ \mathrm{H} & 2.96423700 & -1.28902800 & -2.04098900 \\ \mathrm{H} & 4.63020200 & -1.45163300 & -1.50732500\end{array}$

$\mathrm{Ni}$

$\mathrm{O}$

C

C

$\mathrm{C}$

C

C

C

C

$\mathrm{C}$

$\mathrm{Zn}$

$\mathrm{H}$

$\mathrm{H}$

$\mathrm{H}$

$\mathrm{H}$

$\mathrm{H}$

$\mathrm{H}$

$\mathrm{H}$

$\mathrm{H}$

$\mathrm{H}$

$\mathrm{H}$

$\mathrm{H}$

$\mathrm{H}$

$\mathrm{H}$

$\mathrm{C}$

$\mathrm{H}$

$\mathrm{H}$

$\mathrm{H}$

$\mathrm{Ni}$

$\mathrm{O}$

C

C

C

C

C

\section{Compound TS-37a}

$\mathrm{E}+\mathrm{ZPE}=\mathbf{- 3 7 1 4 . 8 0 0 8 9 8 \text { a.u. }}$

$\begin{array}{llll}0.40123800 & -0.23180000 & -0.20072300\end{array}$

$\begin{array}{llll}3.59338800 & -0.69599400 & -1.13708500\end{array}$

$\begin{array}{llll}3.02430600 & -1.19911800 & -0.17636600\end{array}$

$\begin{array}{lll}2.06261500 & -0.52364200 & 0.69761000\end{array}$

$\begin{array}{lll}1.93894500 & 0.94350000 & 0.58333300\end{array}$

$\begin{array}{lll}1.82513700 & 1.71661400 & 1.89177700\end{array}$

$\begin{array}{lll}0.53783000 & 1.70989600 & -0.43476700\end{array}$

$\begin{array}{llll}-0.68034900 & 1.28254900 & -0.71127100\end{array}$

$\begin{array}{llll}-1.72914300 & 2.00809100 & -1.51588800\end{array}$

$\begin{array}{lll}-0.27611800 & -2.05454700 & -0.40969900\end{array}$

$\begin{array}{lll}-1.79750900 & -0.37392400 & 0.19607300\end{array}$

$\begin{array}{lll}3.20334200 & -2.26938500 & 0.07308600\end{array}$

$\begin{array}{lll}1.91042000 & -0.98113700 & 1.67588100\end{array}$

$\begin{array}{llll}2.71345700 & 1.33057600 & -0.07730100\end{array}$

$\begin{array}{lll}1.02194900 & 1.31971100 & 2.52132000\end{array}$

$\begin{array}{lll}2.76755200 & 1.62528000 & 2.44461600\end{array}$

$\begin{array}{llll}-1.94976400 & 1.45590100 & -2.43814400\end{array}$

$\begin{array}{lll}-2.67832300 & 2.10306500 & -0.97335200\end{array}$

$\begin{array}{llll}-1.40099000 & 3.01598100 & -1.79717400\end{array}$

$\begin{array}{lll}0.39622200 & -2.40917200 & -1.20393500\end{array}$

$\begin{array}{lll}-1.28151000 & -2.30863800 & -0.79667500\end{array}$

$\begin{array}{lll}1.63088800 & 2.78236600 & 1.73116800\end{array}$

$\begin{array}{llll}0.99924300 & 2.67164100 & -0.62825000\end{array}$

$\begin{array}{lll}-0.12003300 & -2.70305500 & 0.45979400\end{array}$

$\begin{array}{lll}-3.59688500 & -0.61154200 & 0.87369100\end{array}$

$\begin{array}{lll}-4.30412600 & -0.87244300 & 0.07721000\end{array}$

$\begin{array}{lll}-3.63516100 & -1.41505900 & 1.61854200\end{array}$

$\begin{array}{lll}-3.96767200 & 0.29963900 & 1.35741400\end{array}$

\section{Compound TS-37b}

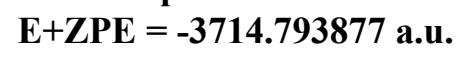

$\begin{array}{lll}0.63985600 & -0.31750100 & -0.20431600\end{array}$

$\begin{array}{lll}-1.29430400 & 2.32763900 & 1.42043500\end{array}$

$\begin{array}{lll}-1.34914900 & 2.02501400 & 0.23748900\end{array}$

$\begin{array}{llll}-0.26681200 & 1.39134700 & -0.54465500\end{array}$

$\begin{array}{lll}1.12911700 & 1.67321900 & -0.03660800\end{array}$

$\begin{array}{llll}2.01113600 & 2.40082100 & -1.04746300\end{array}$

$\begin{array}{llll}2.31193400 & 0.38351500 & 0.65090600\end{array}$ 


$\begin{array}{lccc}\mathrm{C} & 2.20327500 & -0.90922500 & 0.55042400 \\ \mathrm{C} & 2.96519400 & -2.13838400 & 0.88106500 \\ \mathrm{Zn} & -1.56481800 & -0.61372700 & -0.07144600 \\ \mathrm{C} & 0.00110500 & -2.01337400 & -0.96564900 \\ \mathrm{H} & -2.27978400 & 2.22609400 & -0.34158400 \\ \mathrm{H} & -0.40475600 & 1.54857300 & -1.61832200 \\ \mathrm{H} & 1.04571000 & 2.21068900 & 0.90677900 \\ \mathrm{H} & 2.09800500 & 1.83216900 & -1.97960700 \\ \mathrm{H} & 1.56970700 & 3.37649600 & -1.28340000 \\ \mathrm{H} & 3.16906800 & -2.71924800 & -0.02685400 \\ \mathrm{H} & 3.92352700 & -1.92012100 & 1.36934200 \\ \mathrm{H} & 2.38317700 & -2.78946000 & 1.54415600 \\ \mathrm{H} & 0.80111000 & -2.12043800 & -1.71230000 \\ \mathrm{H} & 0.06464500 & -2.88348600 & -0.30269100 \\ \mathrm{H} & 3.03571600 & 1.06350900 & 1.08222400 \\ \mathrm{H} & 3.02308200 & 2.57364000 & -0.66514500 \\ \mathrm{H} & -0.92508300 & -2.15223000 & -1.55414600 \\ \mathrm{C} & -3.44403100 & -0.91703400 & 0.27871000 \\ \mathrm{H} & -3.82578500 & -0.20672400 & 1.01973500 \\ \mathrm{H} & -4.04769400 & -0.80716300 & -0.63053200 \\ \mathrm{H} & -3.61824500 & -1.92697200 & 0.66777100\end{array}$

$\begin{array}{lrrr}\mathrm{Ni} & -0.15540700 & -0.67479500 & -0.23306400 \\ \mathrm{O} & -3.21544400 & -1.50378800 & 0.79302700 \\ \mathrm{C} & -2.57605100 & -1.39592000 & -0.24413900 \\ \mathrm{C} & -1.93190900 & -0.17388800 & -0.76775900 \\ \mathrm{C} & -2.36273000 & 1.16553500 & -0.15451400 \\ \mathrm{C} & -2.44510100 & 2.28995100 & -1.20179800 \\ \mathrm{C} & -1.36575300 & 1.45360600 & 0.92758800 \\ \mathrm{C} & -0.25690200 & 0.69418900 & 0.99213800 \\ \mathrm{C} & 0.69779300 & 0.77392600 & 2.16615900 \\ \mathrm{C} & 1.33286800 & -2.01045800 & -0.08000600 \\ \mathrm{Zn} & 2.02154800 & -0.02556100 & -0.22135400 \\ \mathrm{H} & -2.41377400 & -2.30592600 & -0.88004700 \\ \mathrm{H} & -1.91990500 & -0.19284100 & -1.86876800 \\ \mathrm{H} & -3.35597200 & 1.05233700 & 0.30646900 \\ \mathrm{H} & -1.48063600 & 2.41699400 & -1.70867800 \\ \mathrm{H} & -3.20509500 & 2.06438500 & -1.95945700 \\ \mathrm{H} & 0.71085700 & -0.16602200 & 2.73314900 \\ \mathrm{H} & 1.74134500 & 0.99759900 & 1.89315900 \\ \mathrm{H} & 0.38602900 & 1.56920500 & 2.85613400 \\ \mathrm{H} & 0.64059000 & -2.54508900 & -0.76914500 \\ \mathrm{H} & 1.25302500 & -2.48565000 & 0.90428000 \\ \mathrm{H} & -2.70743900 & 3.24731300 & -0.73655200 \\ \mathrm{H} & -1.56726800 & 2.23936900 & 1.66276300 \\ \mathrm{H} & 2.32346000 & -2.29179500 & -0.47117300 \\ \mathrm{C} & 3.50545300 & 1.17413700 & -0.52854400 \\ \mathrm{H} & 3.42172500 & 1.64974000 & -1.51249300 \\ \mathrm{H} & 3.55066400 & 1.97217500 & 0.22025500 \\ \mathrm{H} & 4.46489200 & 0.64313900 & -0.50244900\end{array}$




\section{Compound TS-38b}

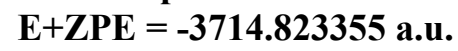

$\mathrm{Ni}$

$\begin{array}{lll}0.10521200 & -1.03502000 & -0.10433600\end{array}$

$\begin{array}{llll}1.57462900 & -1.65718300 & -1.03569300\end{array}$

$\begin{array}{llll}2.68659900 & -1.21671900 & -0.45812300\end{array}$

$\begin{array}{llll}2.90281400 & 0.05978200 & -0.03680200\end{array}$

$\begin{array}{lll}1.87020300 & 1.16479500 & -0.17927700\end{array}$

$\begin{array}{lll}2.51601800 & 2.54264700 & -0.37014300\end{array}$

$\begin{array}{lll}0.96886300 & 1.11595800 & 1.03483800\end{array}$

$\begin{array}{lll}-0.16369300 & 0.36226800 & 1.13449800\end{array}$

$\begin{array}{lll}-0.89431300 & 0.25798500 & 2.46200800\end{array}$

$\begin{array}{lll}-1.33328000 & -2.13134800 & 0.39809300\end{array}$

$\begin{array}{llll}-1.57921400 & 0.47461400 & -0.38290300\end{array}$

$\begin{array}{lll}3.49768300 & -1.94865700 & -0.37814500\end{array}$

$\begin{array}{llll}3.86282300 & 0.30840300 & 0.40847000\end{array}$

$\begin{array}{llll}1.26020400 & 0.94044000 & -1.07151900\end{array}$

$\begin{array}{lll}3.16298800 & 2.54201100 & -1.25351100\end{array}$

$\begin{array}{lll}3.13316200 & 2.80600400 & 0.49818000\end{array}$

$\begin{array}{lll}-1.98684100 & 0.30239400 & 2.35907800\end{array}$

$\begin{array}{lll}-0.67497300 & -0.69385100 & 2.96128400\end{array}$

$\begin{array}{lll}-0.59785600 & 1.06956200 & 3.13776700\end{array}$

$\begin{array}{lll}-2.27615800 & -1.69893200 & 0.75881900\end{array}$

$\begin{array}{lll}-0.95099300 & -2.77601300 & 1.20308100\end{array}$

$\begin{array}{lll}1.75948600 & 3.32640700 & -0.49363200\end{array}$

$\begin{array}{llr}1.31824800 & 1.66984000 & 1.91280600\end{array}$

$\begin{array}{llll}-3.16199400 & 1.27673400 & -1.14725100\end{array}$

$\begin{array}{lll}-1.55979600 & -2.76370000 & -0.47258300\end{array}$

$\begin{array}{llll}-2.98522000 & 2.32371400 & -1.41757600\end{array}$

$\begin{array}{lll}-3.48213200 & 0.74762500 & -2.05150200\end{array}$

$\begin{array}{llll}-3.99447600 & 1.25174600 & -0.43403400\end{array}$

\section{Compound TS-38c}

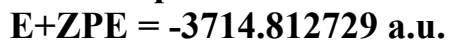

$\mathrm{Ni}$

$\mathrm{O}$

C

C

C

C

C

C

$\mathrm{C}$

$\mathrm{Zn}$

$\mathrm{C}$

C

$\mathrm{H}$

$\mathrm{H}$

$\mathrm{H}$

H

$\mathrm{H}$

$\mathrm{H}$

$\mathrm{H}$

$\mathrm{H}$

$\mathrm{H}$

$\mathrm{H}$ $\begin{array}{lll}0.54708400 & 0.19081600 & 0.59837700\end{array}$

$\begin{array}{llll}-0.24374100 & -1.98731100 & -0.62027800\end{array}$

$\begin{array}{lll}0.64256800 & -1.94218300 & 0.36186400\end{array}$

$\begin{array}{lll}1.86400400 & -1.26284700 & 0.33360400\end{array}$

$\begin{array}{lll}2.15712900 & -0.26687500 & -0.70670900\end{array}$

$\begin{array}{llll}3.61840100 & 0.15031700 & -0.75985500\end{array}$

$\begin{array}{llll}1.28949500 & 1.51957900 & -0.71706600\end{array}$

$\begin{array}{llll}0.17683200 & 1.83450400 & -0.12617200\end{array}$

$\begin{array}{lll}-0.73303000 & 2.99922500 & 0.02523500\end{array}$

$\begin{array}{lll}-1.50950200 & -0.57550800 & -0.36482500\end{array}$

$\begin{array}{lll}-0.74898600 & 0.08909700 & 2.03375400\end{array}$

$\begin{array}{llll}-3.27889700 & 0.05293200 & -0.84092900\end{array}$

$\begin{array}{lll}0.47581500 & -2.61770700 & 1.21027200\end{array}$

$2.57437500 \quad-1.42863200 \quad 1.14011600$

$\begin{array}{lll}1.76112000 & -0.55933800 & -1.67970100\end{array}$

$\begin{array}{llll}3.95618000 & 0.54800700 & 0.20321500\end{array}$

$\begin{array}{lll}4.23521800 & -0.72348000 & -1.00087500\end{array}$

$\begin{array}{llll}3.80869300 & 0.90882800 & -1.52589800\end{array}$

$\begin{array}{lll}1.98408200 & 1.97533800 & -1.40842500\end{array}$

$\begin{array}{llll}-0.71511100 & 3.35788200 & 1.06135900\end{array}$

$\begin{array}{llll}-0.45258700 & 3.83187100 & -0.63121800\end{array}$

$\begin{array}{llll}-1.76988100 & 2.72004200 & -0.19295600\end{array}$ 
S 55

$\begin{array}{rrrr}\mathrm{H} & -1.46534300 & 0.91713700 & 2.07979100 \\ \mathrm{H} & -0.13496200 & 0.15288300 & 2.94536800 \\ \mathrm{H} & -1.32716100 & -0.84338700 & 2.14650100 \\ \mathrm{H} & -3.68557100 & 0.72606100 & -0.07760800 \\ \mathrm{H} & -3.27274500 & 0.59565000 & -1.79330200 \\ \mathrm{H} & -3.98058100 & -0.78278900 & -0.94657300\end{array}$

